Supporting Information

\title{
Seeded Self-assembly of Charge-terminated Poly(3-hexylthiophene) Amphiphiles based on the Energy Landscape
}

Authors: Tomoya Fukui ${ }^{1, \dagger}$, J. Diego Garcia-Hernandez ${ }^{1}$, Liam R. MacFarlane ${ }^{1}$, Shixing Lei ${ }^{1}$, George R. Whittell ${ }^{2}$, and Ian Manners ${ }^{1 *}$

\author{
Affiliations: \\ ${ }^{1}$ Department of Chemistry, University of Victoria, Victoria, Bristish Columbia V8P 5C2, \\ Canada \\ ${ }^{2}$ School of Chemistry, University of Bristol, Cantock's Close, Bristol BS8 1TS, UK \\ †Present address: Tokyo Institute of Technology, R1-1, 4259 Nagatsuta, Midori-ku, Yokohama, \\ Kanagawa, 226-8503, Japan
}

*Correspondence to: imanners@uvic.ca

Table of Contents

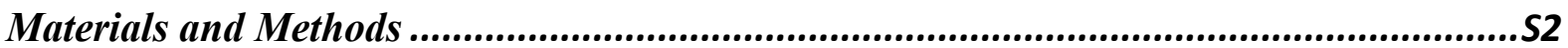

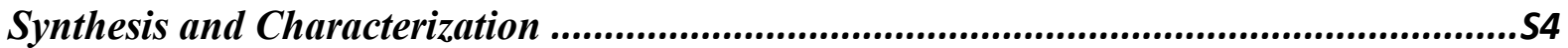

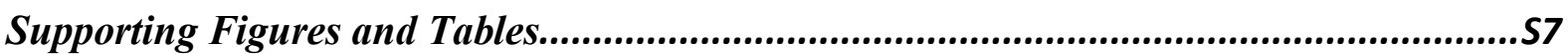

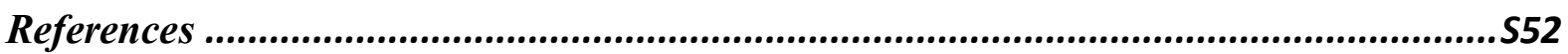




\section{Materials and Methods}

Unless otherwise noted, reagents and solvents were purchased from commercial suppliers and used without further purification. All reactions were conducted under nitrogen atmosphere by using dry solvents. (4-bromophenyl)diphenyl phosphine was synthesized according to reported procedure ${ }^{\mathrm{S} 1}$.

\section{Variable-temperature UV/vis absorption spectroscopy (VT-UV)}

VT-UV spectra were recorded using a quartz cuvette of $1.0 \mathrm{~cm}$ path length for the solution samples on a Cary 100 spectrometer equipped with a Peltier temperature controller. Experiments were conducted at a concentration of $0.02 \mathrm{mg} / \mathrm{mL}$ to allow convenient monitoring of fiber-like micelle formation. At higher concentrations the $T_{\mathrm{c}}$ values were found to be outside the range of the instrument $\left(>100{ }^{\circ} \mathrm{C}\right)$.

\section{Photoluminescence spectroscopy (PL)}

PL spectra were recorded using a quartz cuvette of $1.0 \mathrm{~cm}$ path length for the solution samples on a PTI QM40 fluorometer.

\section{Transmission electron microscopy (TEM)}

Samples were prepared by drop-casting method. One drop $(10 \mu \mathrm{L})$ of the solution onto a carbon-coated copper $(\mathrm{Cu})$ grid which was placed on a filter paper to remove solvent. Bright field TEM images were obtained on a JEOL1011 microscope operating at $120 \mathrm{kV}$ and equipped with $11 \mathrm{M}$ pixel CCD camera. All TEM images were obtained without staining.

Length distribution of fiber-like micelles were determined by using ImageJ from the U.S. National Institute of Health. For each sample, at least 100 micelles from several images were traced by hand in order to obtain the length information. The number-average length $\left(L_{\mathrm{n}}\right)$, weight-average length $\left(L_{\mathrm{w}}\right)$, and polydispersity index $(\bigoplus)$ were calculated by using equations 1-3 from the measurements of the contour length $\left(L_{\mathrm{i}}\right)$ of individual micelles, where $N_{\mathrm{i}}$ is the number of the fibers of length $L_{\mathrm{i}}$, and $n$ is the number of micelles examined in each sample.

$$
\begin{array}{cc}
L_{n}=\frac{\sum_{i=1}^{n} N_{i} L_{i}}{\sum_{i=1}^{n} N_{i}} & \text { Eq. } 1 \\
L_{w}=\frac{\sum_{i=1}^{n} N_{i} L_{i}^{2}}{\sum_{i=1}^{n} N_{i} L_{i}} & \text { Eq. } 2 \\
Đ=\frac{L_{w}}{L_{n}} & \text { Eq. 3 }
\end{array}
$$

We also checked TEM images of all solution samples prepared after a month. No significant changes in the length and dispersity of fiber-like micelles were observed. 


\section{Confocal laser scanning microscopy (CLSM)}

CLSM images were obtained by using a ZEISS LSM 880, Axio Observer device equipped with an inverted epifluorescence microscope. Images were recorded by using a PlanApochromat $63 \times / 1.4$ oil immersion objective and a gallium arsenide phosphide (GaAsP) detector. Samples were excited with an Argon laser $\left(\lambda_{\mathrm{ex}}=514 \mathrm{~nm}\right)$ at observation windows of $580-660 \mathrm{~nm}$ in channel mode or from 570-720 nm with $10 \mathrm{~nm}$ intervals in lambda mode. The resulting images were obtained as digital false-colour images and then color coded as pinkishpurple. Samples were prepared by placing $10 \mu \mathrm{L}$ of diluted micelle solution (benzonitrile, 0.01 $\mathrm{mg} / \mathrm{mL}$ ) on a glass slide, a cover slip was then placed on top of the solution and sealed in place using fast-dry nail polish.

\section{Scanning electron microscopy (SEM)}

TEM samples were used for SEM imaging without staining. SEM images were obtained by a Hitachi S-4800 field emission scanning electron microscope operating at $10 \mathrm{kV}$ and equipped with cold field emission electron source, ExB filter technology, and ring-type YAG backscatter detector for high resolution.

\section{Atomic force microscopy (AFM)}

Samples were prepared by spin-coat method. One drop of the solution (ca. $5 \mu \mathrm{L}$ ) was dropped onto the mica substrate under the spinning at 3000 r.p.m.. AFM images were obtained by Agilent Technologies 5500 Scanning Probe Microscope operating by tapping-mode. Obtained images were analysed by using a Gwyddion software. 


\section{Synthesis and Characterization}

Scheme 1. Synthesis of $\left[P T_{n} P\right] I(n=19,30,50)$.

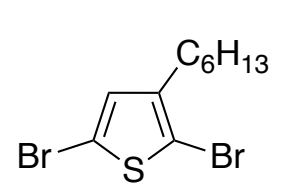

1) $i-\mathrm{PrMgCl} \cdot \mathrm{LiCl}$

2) $\mathrm{Ni}(o$-tolyl)(dppe)Cl

THF<smiles>Cc1ccccc1C(C)(C)c1cc(C)c([N+](Br)(Br)[I-]2CCCC2)s1</smiles><smiles>COc1ccc(P(c2ccccc2)c2ccccc2)cc1</smiles><smiles>Cc1ccccc1C(C)(C)c1cc(C)c(C(C)(C)c2ccc(P(c3ccccc3)c3ccccc3)cc2)s1</smiles>

$\frac{\mathrm{CH}_{3} \mathrm{I}}{\mathrm{THF}}$<smiles>CCCCCc1cc(C(C)(C)c2ccccc2C)sc1C(C)(C)c1ccc([PH](C)(c2ccccc2)c2ccccc2)cc1</smiles>

$\left[\mathrm{PT}_{\mathrm{n}} \mathrm{P}\right] \mathbf{I}$ 


\section{Experimental procedures for the synthesis of polymers}

\section{Preparation of chloro 4-(diphenyphosphino)phenyl magnesium}

In the glovebox under $\mathrm{N}_{2}$ atmosphere, (4-bromophenyl)diphenyl phosphine (512 $\mathrm{mg}$, $1.50 \mathrm{mmol}$ ) was dispersed into $1.2 \mathrm{~mL}$ of distilled THF. To the solution, iso-propylmagnesium chloride lithium chloride solution $(1.3 \mathrm{M}, 1.2 \mathrm{~mL})$ was added dropwise. The solution was stirred for overnight at room temperature. The colour of the solution turned into brownish-red from colorless. The solution was used as THF solution of chloro 4-(diphenyphosphino)phenyl magnesium lithium chloride (ca. $0.6 \mathrm{M}$ ) without further purification.

\section{Synthesis of $[\mathbf{P 3 H T}]_{n} \mathbf{P P h}_{2}$ (Typical procedure for $[\mathbf{P 3 H T}]_{50} \mathbf{P P h}_{2}$ )}

In the glovebox under $\mathrm{N}_{2}$ atmosphere, 2,5-dibromo-3-hexylthiophene $(608 \mathrm{mg}, 1.86$ mmol) was dispersed into $20 \mathrm{~mL}$ of distilled THF. To the solution, iso-propylmagnesium chloride lithium chloride solution $(1.3 \mathrm{M}, 1.4 \mathrm{~mL})$ was added dropwise. The solution was stirred for $1 \mathrm{~h}$ at room temperature. $\mathrm{Ni}(o$-tolyl $)(\mathrm{dppe}) \mathrm{Cl}(19 \mathrm{mg}, 0.0326 \mathrm{mmol})$ in THF $(1 \mathrm{~mL})$ was added to the solution in one portion. After 8 min of stirring, the THF solution of chloro 4(diphenyphosphino)phenyl magnesium lithium chloride (ca. $0.6 \mathrm{M}, 1 \mathrm{~mL}$ ) was added and the resultant mixture was stirred at room temperature for $2 \mathrm{~h}$. The reaction was quenched by addition of degassed methanol. The formed precipitates were washed by degassed methanol, acetone, and hexane 3 times, respectively. The obtained solid was dried under vacuum at 40 ${ }^{\circ} \mathrm{C}$ for $2 \mathrm{~h}$. [P3HT] $\mathbf{5 0}_{\mathbf{0}} \mathbf{P P h}_{\mathbf{2}}$ was obtained as purple solid (243 mg, Yield: $76 \%$ ).

The obtained solid was used for the next reaction without further purification to avoid the oxidation of phosphine group.

${ }^{1} \mathrm{H}$ NMR (500 MHz, $\left.\mathrm{CDCl}_{3}\right) \delta$ 7.47-7.43 (m, aromatic), 7.37-7.33 (m, aromatic), 7.25-7.23 (m, aromatic), $6.98\left(\mathrm{~s}\right.$, thiophene), 2.81(t, $\left.J=7 \mathrm{~Hz},-\mathrm{CH}_{2}-\right), 2.50\left(\mathrm{~s},-\mathrm{CH}_{3}\right), 1.74-1.68(\mathrm{~m}$, $\left.\mathrm{CH}_{2-}\right), 1.46-1.43\left(\mathrm{~m},-\mathrm{CH}_{2}-\right), 1.38-1.34\left(\mathrm{~m},-\mathrm{CH}_{2}-\right), 0.92\left(\mathrm{t}, \mathrm{J}=7 \mathrm{~Hz},-\mathrm{CH}_{3}\right) .{ }^{31} \mathrm{P}$ NMR (202 $\left.\mathrm{MHz}, \mathrm{CDCl}_{3}\right) \delta-5.72$.

Attempts to prepare materials with $\mathrm{DP}_{\mathrm{n}}<20$ and low dispersities $(<1.22)$ were hindered by the low solubility of the Ni catalyst. Further fractionation of the $\left[\mathbf{P T} \mathbf{T}_{19} \mathbf{P}\right] \mathbf{I}$ sample with biobeads was unsuccessful.

Synthesis of $\left[\mathbf{P T}_{\mathbf{n}} \mathbf{P}\right] \mathbf{I}$ (Typical procedure for $\left[\mathbf{P T}_{\mathbf{5 0}} \mathbf{P}\right] \mathbf{I}$ )

[P3HT] 50 PPh $_{2}(215 \mathrm{mg})$ was dissolved in degassed THF (24 mL) under $\mathrm{N}_{2}$ atmosphere. To the solution, degassed methyl iodide $(11 \mathrm{~mL}, 177 \mathrm{mmol})$ was added and the solution was heated at $40{ }^{\circ} \mathrm{C}$ for $24 \mathrm{~h}$. The resultant solution was poured into hexane and the formed precipitates were collected by centrifuge. The obtained solid was dissolved in THF (concentration was ca. $4 \mathrm{mg} / \mathrm{mL}$ ) and then poured into hexane slowly (volume ratio of hexane to THF was 1). The formed precipitates were collected by centrifuge. This process was repeated 20 times to remove non-chaeged homopolymers. The resulting solid was dried at $40{ }^{\circ} \mathrm{C}$ under vacuum for $24 \mathrm{~h}$. [PT $\mathbf{5 0} \mathbf{P}] \mathbf{I}$ was obtained as purple solid (183 $\mathrm{mg}$, Yield: $83 \%$ ).

${ }^{1} \mathrm{H}$ NMR $\left(500 \mathrm{MHz}, \mathrm{CDCl}_{3}\right) \delta$ 7.86-7.71 (m, $\left.-\mathrm{C}_{6} \mathrm{H}_{4}-\mathrm{P}-\left(\mathrm{C}_{6} \mathrm{H}_{5}\right)_{2}\right), 7.47-7.43$ (m, tolyl), $6.98(\mathrm{~s}$, thiophene), 3.27 (d, $\left.J=12 \mathrm{~Hz}, \mathrm{P}-\mathrm{CH}_{3}\right), 2.81\left(\mathrm{t},-\mathrm{CH}_{2^{-}}\right), 2.49\left(\mathrm{~s},-\mathrm{CH}_{3}\right), 1.77-1.71\left(\mathrm{~m},-\mathrm{CH}_{2^{-}}\right)$, 1.47-1.41 (m, $\left.-\mathrm{CH}_{2^{-}}\right), 1.38-1.32\left(\mathrm{~m},-\mathrm{CH}_{2^{-}}\right), 0.92\left(t, J=7 \mathrm{~Hz},-\mathrm{CH}_{3}\right) .{ }^{31} \mathrm{P}$ NMR $(202 \mathrm{MHz}$, $\left.\mathrm{CDCl}_{3}\right) \delta$ 21.6. GPC: [PT $\left.{ }_{19} P\right] \mathbf{I}: M_{\mathrm{n}}=3661 \mathrm{Da}, M_{\mathrm{w}}=4468 \mathrm{Da}$, PDI $\left(M_{\mathrm{w}} / M_{\mathrm{n}}\right)=1.22$. [PT 30 P]I: $M_{\mathrm{n}}=5896 \mathrm{Da}, M_{\mathrm{w}}=6304 \mathrm{Da}$, PDI $\left(M_{\mathrm{w}} / M_{\mathrm{n}}\right)=1.07$. [PT ${ }_{50}$ P]I: $M_{\mathrm{n}}=8453 \mathrm{Da}, M_{\mathrm{w}}=8828 \mathrm{Da}$, PDI $\left(M_{\mathrm{w}} / M_{\mathrm{n}}\right)=1.04$. 
The number-average degree of polymerization $\left(\mathrm{DP}_{\mathrm{n}}\right)$ was determined by using both ${ }^{1} \mathrm{H}$ NMR and MALDI-TOF-MS. To evaluate DP ${ }_{n}$ based on NMR analysis, we used a peak at 2.49 ppm ( $\mathrm{CH}_{3}$ - group on tolyl group) as a standard, and calculated the ratio of integrals of each peaks. The obtained ratio was consistent with the value obtained by MALDI-TOF-MS.

\section{Experimental procedures for the self-assembly}

\section{Preparation of fiber-like micelles of $\left[\mathrm{PT}_{\mathrm{n}} \mathrm{P}\right] \mathrm{I}$ in benzonitrile}

Benzonitrile solution of $\left[\mathbf{P} \mathbf{T}_{\mathbf{n}} \mathbf{P}\right] \mathbf{I}(\mathrm{n}=19,30,50$, Conc. $=0.5 \mathrm{mg} / \mathrm{mL})$ was dissolved at $100{ }^{\circ} \mathrm{C}$ for $1 \mathrm{~h}$. Subsequently, the hot solution was cooled to room temperature slowly. The solution was aged under stirring at room temperature for $12 \mathrm{~h}$.

\section{Preparation of seed micelles of $\left[\mathrm{PT}_{\mathbf{n}} \mathrm{P}\right] \mathrm{I}$}

The solution of the polydisperse fiber-like micelles (Conc. $=0.5 \mathrm{mg} / \mathrm{mL}$ ) was diluted by the addition of benzonitrile (Conc. $=0.1 \mathrm{mg} / \mathrm{mL}$ ) and subsequently degassed by $\mathrm{N}_{2}$ for $15 \mathrm{~min}$. The polydisperse fiber-like micelles were fragmented by applying the sonication under $\mathrm{N}_{2}$ at $10{ }^{\circ} \mathrm{C}$ for $2 \mathrm{~h}$ to yield short micelles. The short micelles were thermally annealed at $40{ }^{\circ} \mathrm{C}$ for $30 \mathrm{~min}$ and then cooled to room temperature. The length of resulting seeds was evaluated by TEM analysis.

\section{Living CDSA for $\left[\mathrm{PT}_{\mathrm{n}} \mathrm{P}\right] \mathrm{I}$}

The solution of the seeds (Conc. $=0.1 \mathrm{mg} / \mathrm{mL}$ ) was diluted by benzonitrile or benzonitrile/THF mixture to the appropriate concentration. The solution of seed micelles was warmed at $35^{\circ} \mathrm{C}$ for $5 \mathrm{~min}$. To the seed micelles, appropriate amounts of unimers in THF were added (final Conc. $=0.2 \mathrm{mg} / \mathrm{mL}$ ). After the mechanical shaking for $10 \mathrm{sec}$, the solutions were aged for $12 \mathrm{~h}$. TEM analyses evaluated the length of the resulting micelles. Note that the concentration of the resulting micelles was too high for the TEM analysis. Therefore, the solution was diluted to $0.02 \mathrm{mg} / \mathrm{mL}$ by using benzonitrile or benzonitrile/THF mixture.

\section{Kinetic studies of the living CDSA process}

Kinetic studies of living CDSA process shown in Figure S22-25, S32, and S33 were performed by using a Cary 100 spectrometer equipped with a Peltier temperature controller. $\mathrm{UV}$-vis analysis in solution avoids the potential drying and aggregation effects that can complicate the use of the alternative method involving TEM analysis of fiber growth. The seed micelles used were as shown in Figure S18. It is noteworthy that the fiber-like micelles formed by living CDSA were colloidally stable in benzonitrile or benzonitrile/THF solution. The length and dispersity of fiber-like micelles was unchanged after a month based on TEM analysis, which indicated that dynamic exchange of unimers at the termini of micelles is negligible on the timescale of the kinetic studies. 


\section{Supporting Figures and Tables}

Characterization of polymers

a

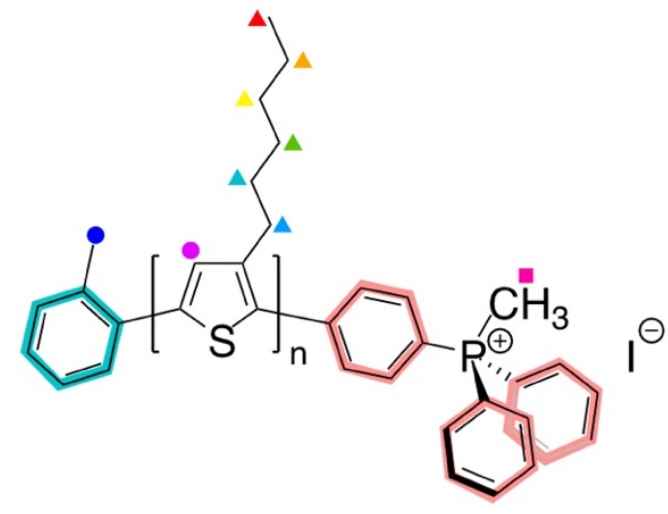

b
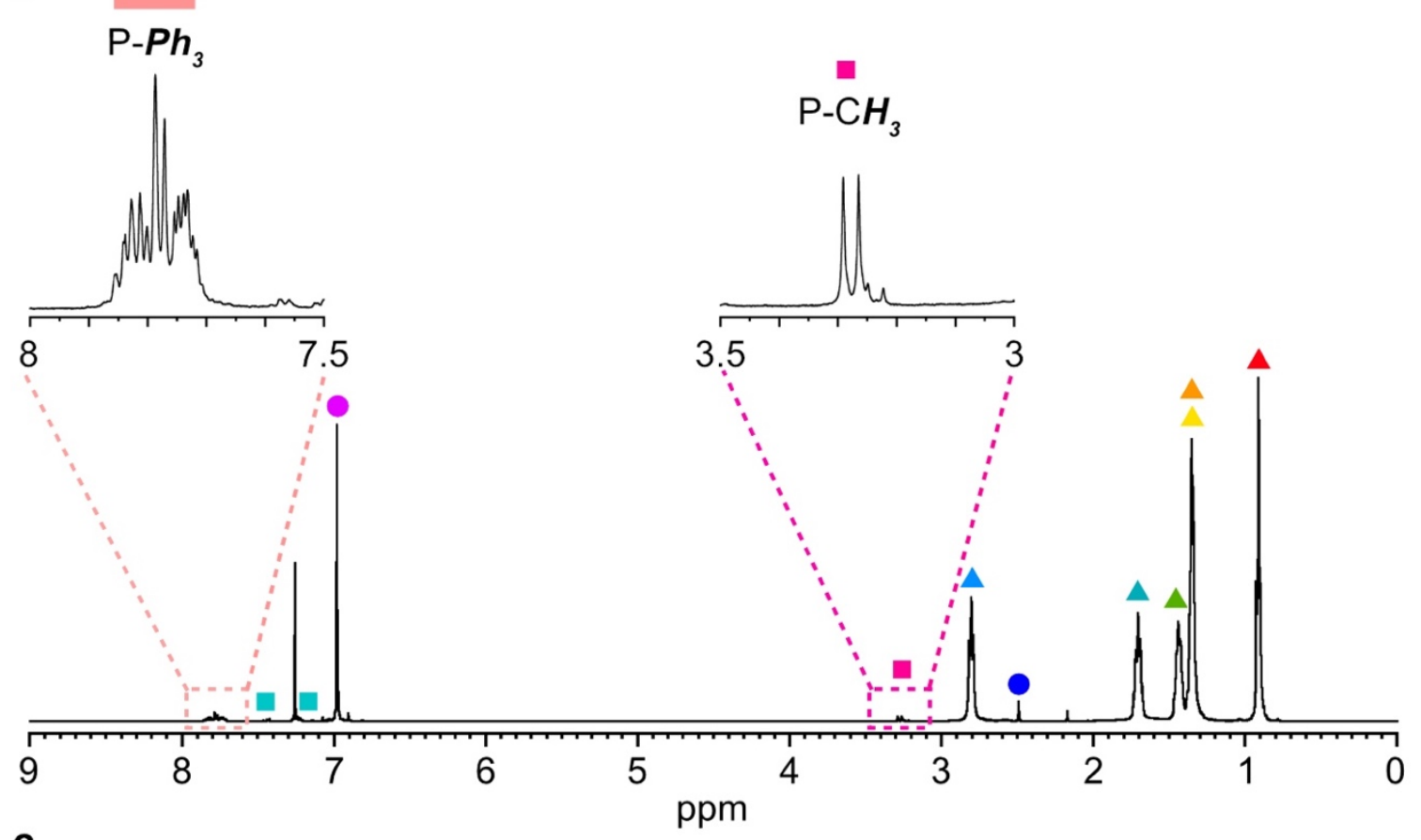

C

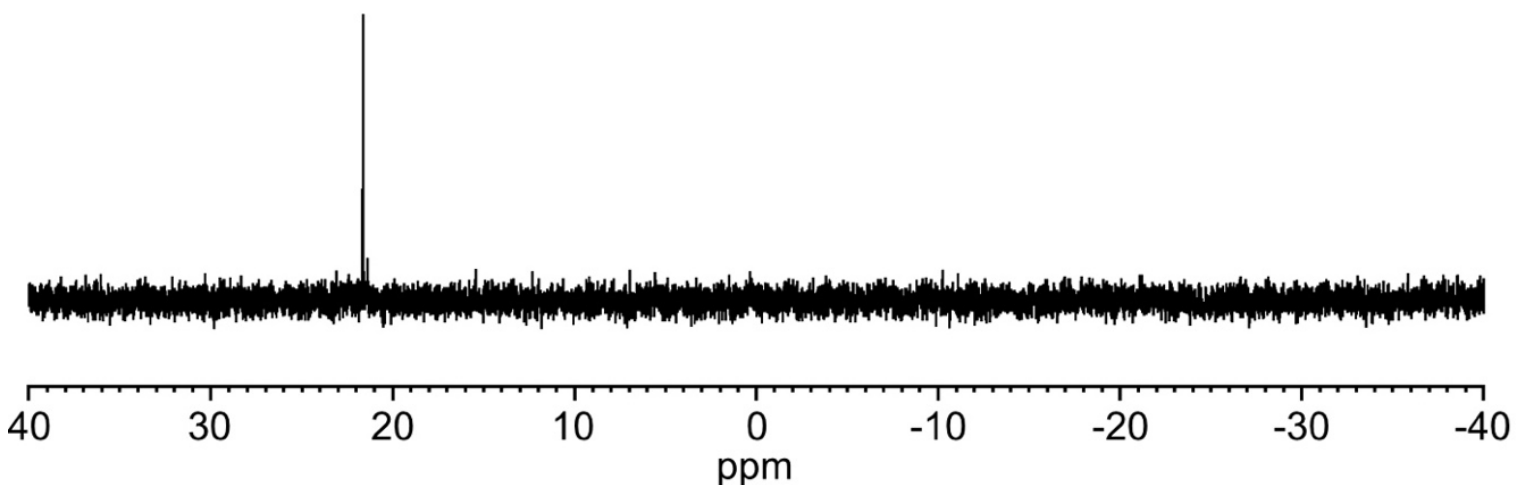

Figure S1.

(a) Polymer structure of $[\mathbf{P T} \mathbf{n} \mathbf{P}] \mathbf{I}(\mathbf{b}$ and $\mathbf{c})$ NMR spectrum of $[\mathbf{P T} \mathbf{n} \mathbf{P}] \mathbf{I}$ in $\mathrm{CDCl}_{3}:(\mathbf{b}){ }^{1} \mathrm{H}(\mathbf{c}){ }^{31} \mathbf{P}$. 
Characterization of polymers

a

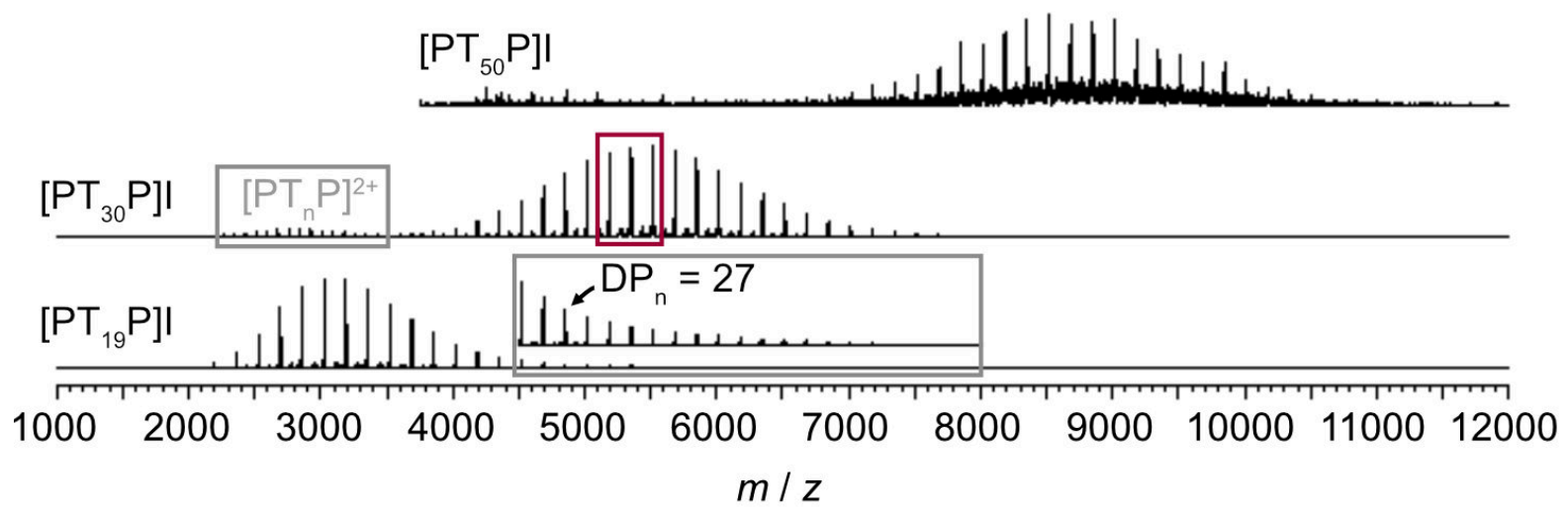

b

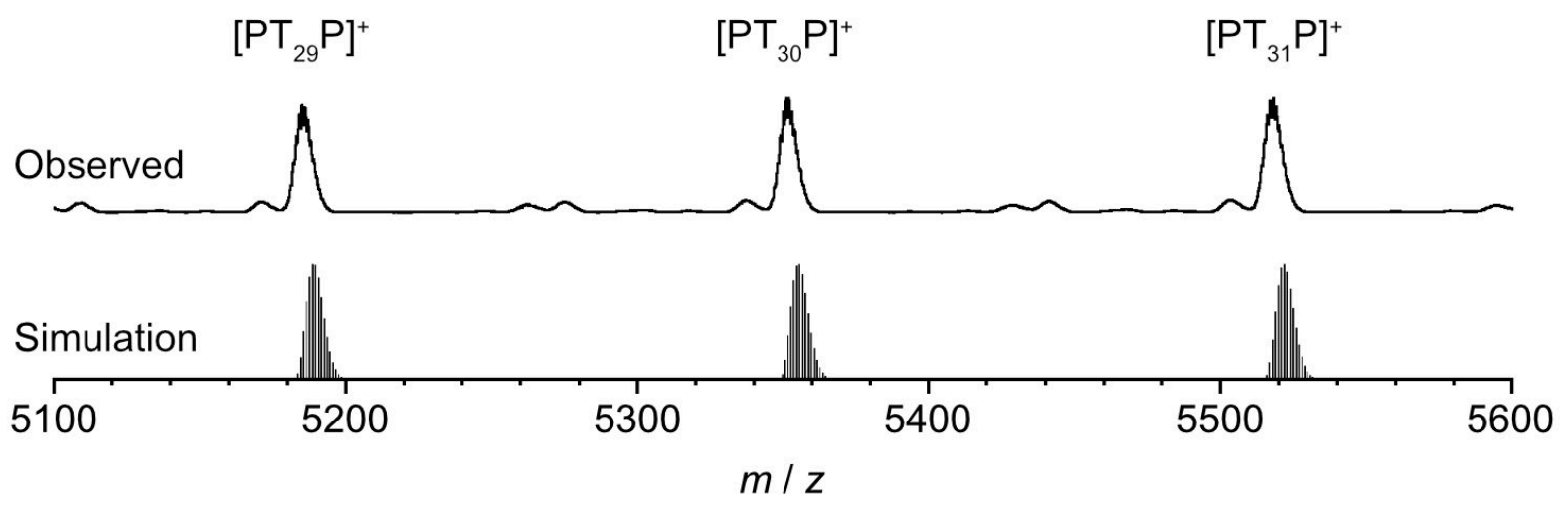

Figure S2.

(a) MALDI-TOF mass spectrum of $\left[\mathbf{P} \mathbf{T}_{\mathbf{1 9}} \mathbf{P}\right] \mathbf{I}$, $\left[\mathbf{P T}_{\mathbf{3 0}} \mathbf{P}\right] \mathbf{I}$, and $\left[\mathbf{P T} \mathbf{5 0}_{\mathbf{5 0}} \mathbf{P}\right] \mathbf{I}$. (b) Expanded MALDITOF mass spectra of $\left[\mathbf{P T}_{\mathbf{3 0}} \mathbf{P}\right] \mathbf{I}$ in the circled range in Figure $\mathrm{S} 2 \mathrm{a}$ and simulated peaks.

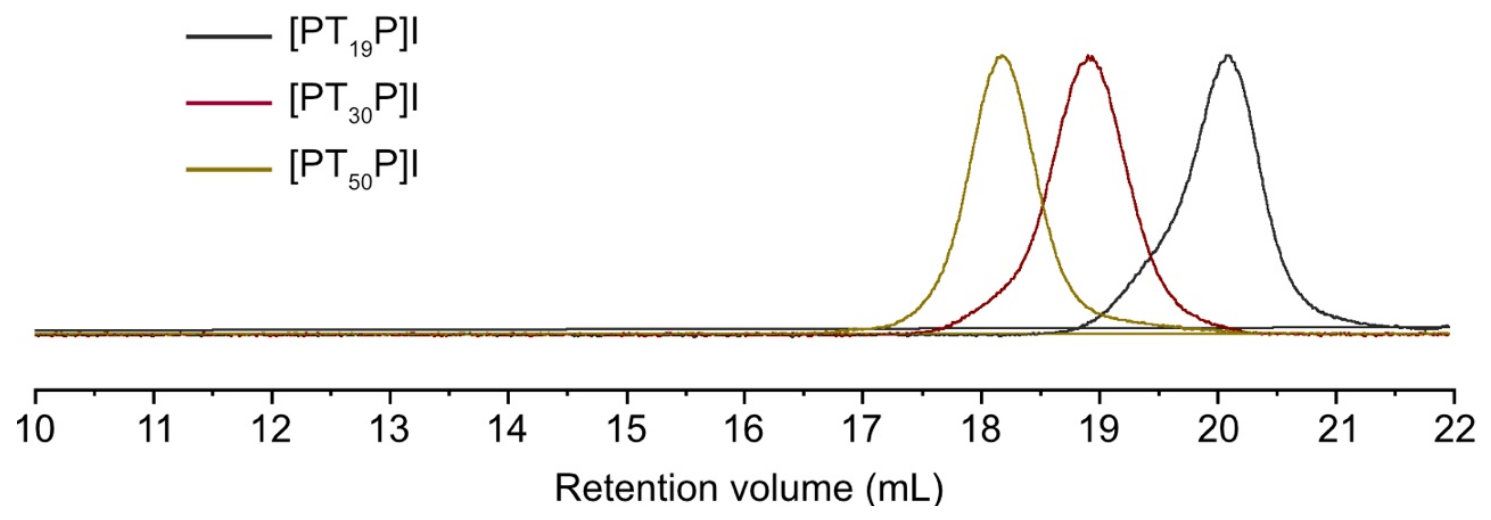

Figure S3.

GPC trace of $\left[\mathbf{P T} \mathbf{T}_{\mathbf{n}} \mathbf{P}\right] \mathbf{I}(\mathrm{n}=19,30$, and 50) using THF as eluent (UV response at $254 \mathrm{~nm})$. PDIs $\left(M_{\mathrm{w}} / M_{\mathrm{n}}\right)$ of $\left[\mathbf{P} \mathbf{T}_{19} \mathbf{P}\right] \mathbf{I},\left[\mathbf{P} \mathbf{T}_{\mathbf{3 0}} \mathbf{P}\right] \mathbf{I}$, and $\left[\mathbf{P T} \mathbf{T}_{\mathbf{5 0}} \mathbf{P}\right] \mathbf{I}$ were found to be $1.22,1.07$, and 1.04, respectively. 
Self-assembly of $[\mathbf{P 3 H T}]_{30} \mathbf{P P h}_{2}$

a

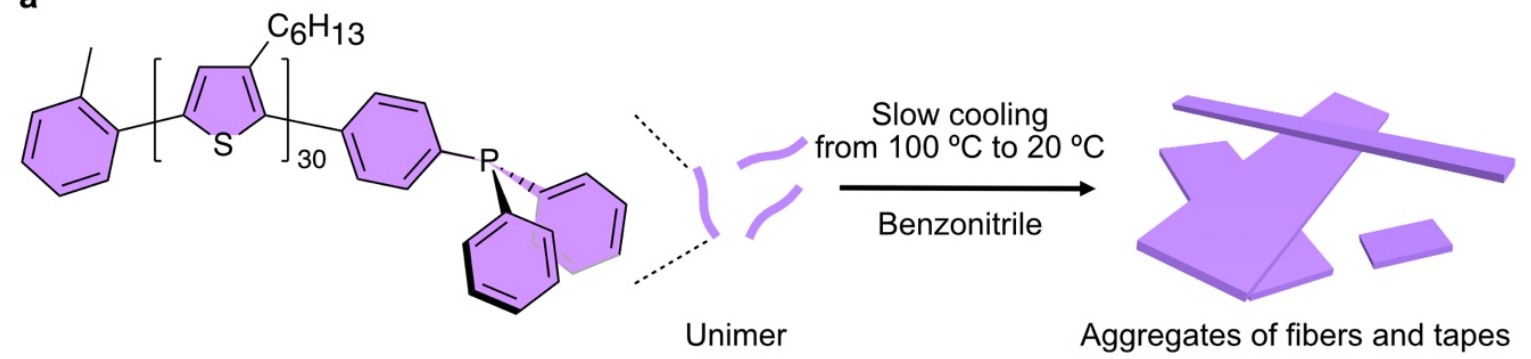

b
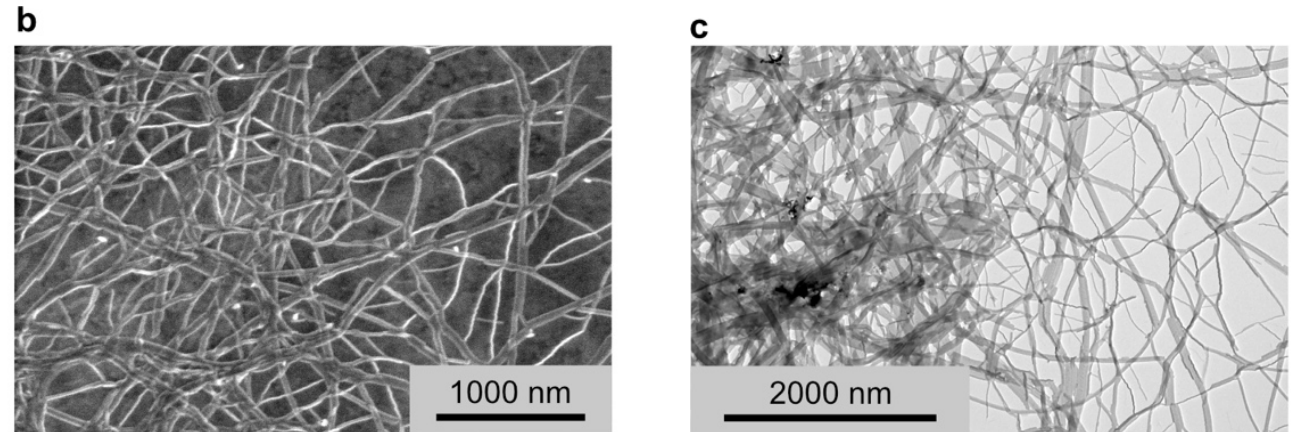

Figure S4.

(a) Schematic representation of self-assembly of $[\mathbf{P 3 H T}]_{30} \mathbf{P P h} \mathbf{h}_{2}$ in benzonitrile $(0.5 \mathrm{mg} / \mathrm{mL})$.

(b) SEM image of the resulting precipitate. (c) TEM image of the resulting precipitate. 


\section{Self-assembly of $\left[\mathbf{P T} \underline{\mathbf{T}_{19}} \underline{\mathbf{P}] \mathbf{I}}\right.$}

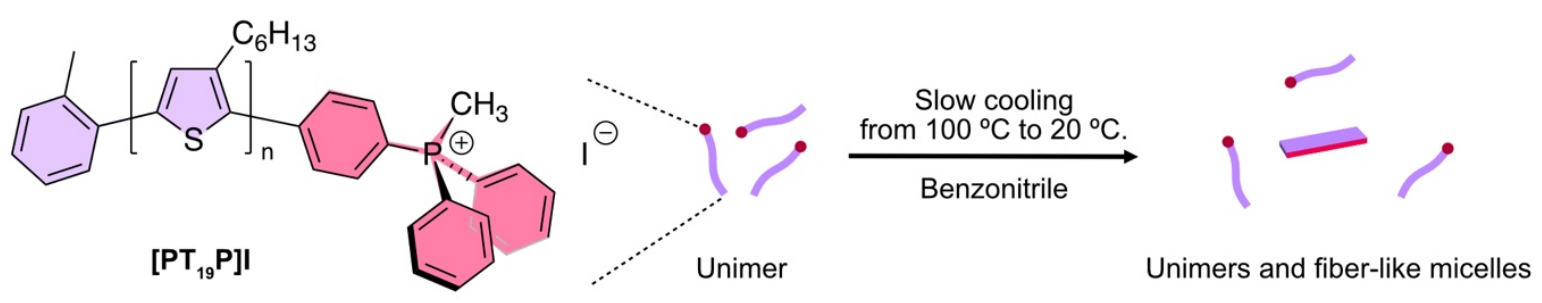

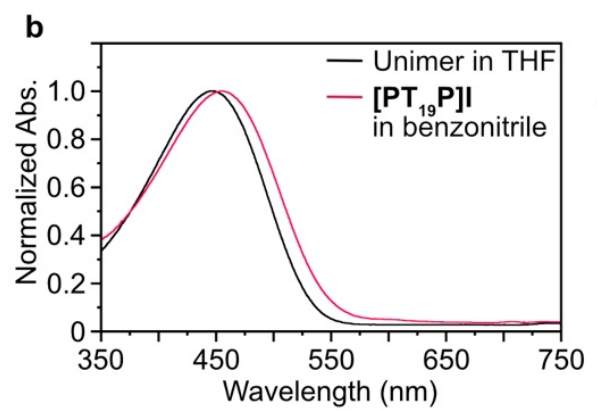

d

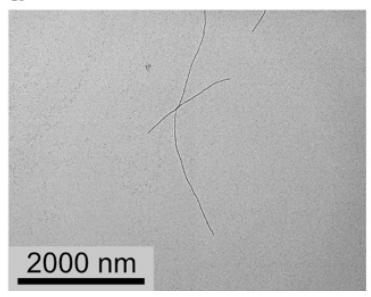

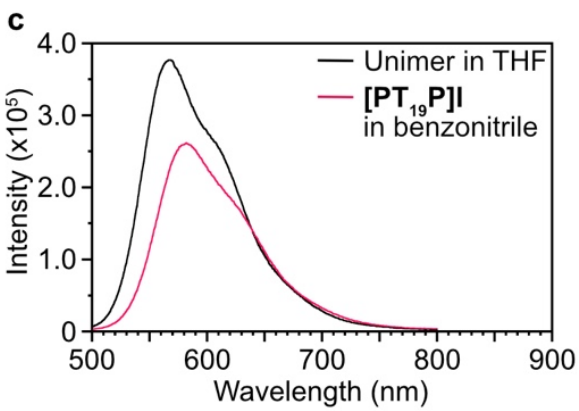

e

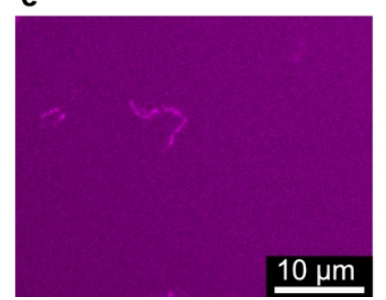

Figure S5.

(a) Schematic representation of self-assembly of [PT $\left.{ }_{19} \mathbf{P}\right] \mathbf{I}$ in benzonitrile. (b) Absorption spectra of $\left[\mathbf{P} \mathbf{T}_{19} \mathbf{P}\right] \mathbf{I}$ in THF (black) or benzonitrile (red) (Conc. $=0.02 \mathrm{mg} / \mathrm{mL}$ ). (c) Photoluminescence spectra of [PT $\left.{ }_{19} \mathbf{P}\right] \mathbf{I}$ in THF (black) or benzonitrile (red) (Conc.: 0.02 $\mathrm{mg} / \mathrm{mL}, \lambda_{\mathrm{ex}}=440 \mathrm{~nm}$ for THF solution, $\lambda_{\mathrm{ex}}=567 \mathrm{~nm}$ for benzonitrile solution). (d) TEM image of the micelles on carbon-coated $\mathrm{Cu}$ grid. (e) CLSM image of micelles of $\left[\mathbf{P T} \mathbf{T}_{19} \mathbf{P}\right] \mathbf{I}$ in benzonitrile (Conc. $=0.02 \mathrm{mg} / \mathrm{mL})$. 


\section{Self-assembly of $[\mathbf{P T} \underline{\mathbf{3 0}} \underline{\mathbf{P}] \mathbf{I}}$}
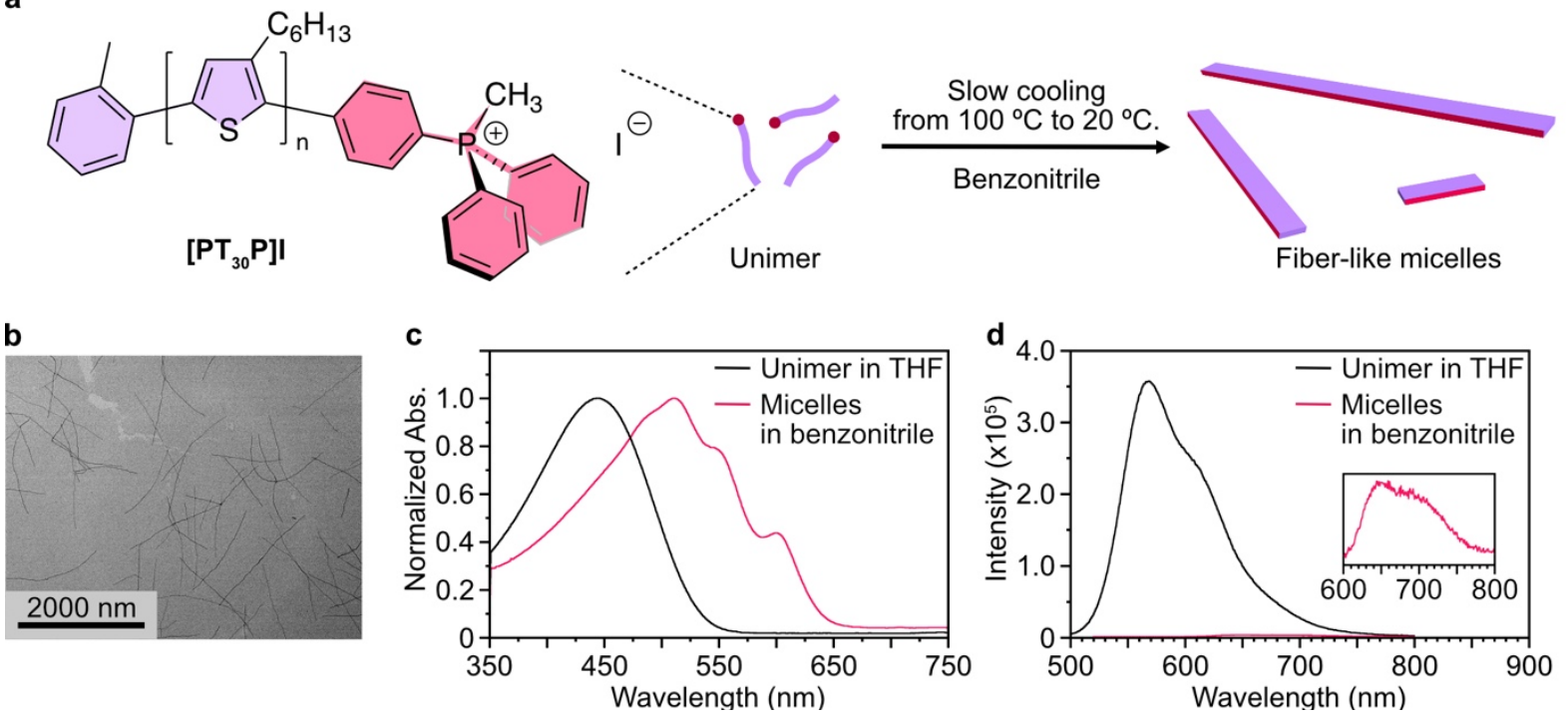

Figure S6.

(a) Schematic representation of self-assembly of $\left[\mathbf{P T}_{\mathbf{3 0}} \mathbf{P}\right] \mathbf{I}$ in benzonitrile. (b) TEM image of the fiber-like micelles of $\left[\mathbf{P} \mathbf{T}_{30} \mathbf{P}\right] \mathbf{I}$ on carbon-coated $\mathrm{Cu}$ grid. $(\mathbf{c})$ Absorption spectra of $\left[\mathbf{P T} \mathbf{T}_{\mathbf{3 0}} \mathbf{P}\right] \mathbf{I}$ in THF (black) or benzonitrile (red) (Conc. $=0.02 \mathrm{mg} / \mathrm{mL})$. (d) Photoluminescence spectra of $\left[\mathbf{P T}_{30} \mathbf{P}\right] \mathbf{I}$ in THF (black) or benzonitrile (red) (Conc.: $0.02 \mathrm{mg} / \mathrm{mL}, \lambda_{\mathrm{ex}}=440 \mathrm{~nm}$ for THF solution, $\lambda_{\mathrm{ex}}=513 \mathrm{~nm}$ for benzonitrile solution). 
a

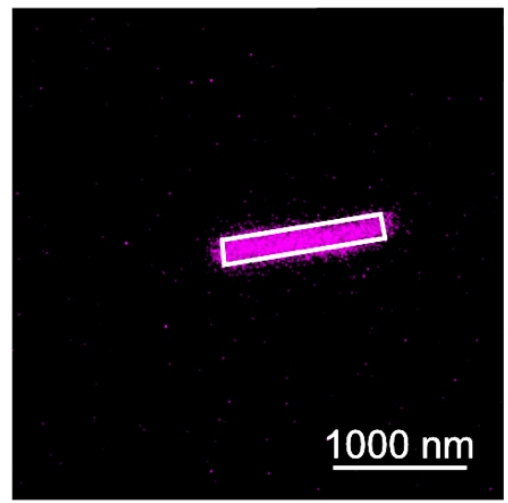

C
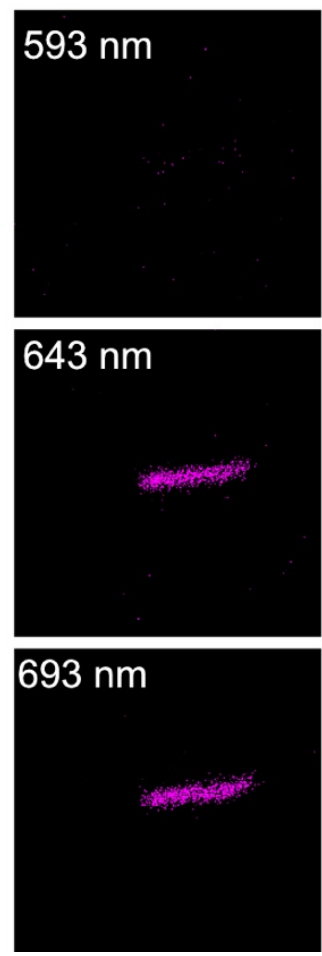

b

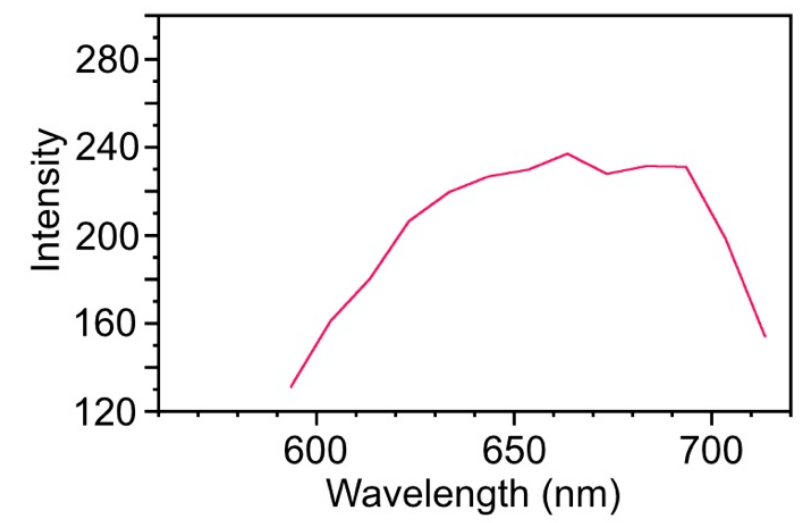

Figure S7.

(a) CLSM image of the fiber-like micelles of $\left[\mathbf{P T}_{\mathbf{3 0}} \mathbf{P}\right] \mathbf{I}$ in benzonitrile (Conc. $\left.=0.02 \mathrm{mg} / \mathrm{mL}\right)$. (b) Photoluminescence spectrum of a single fiber-like micelle of $\left[\mathbf{P T}_{\mathbf{3 0}} \mathbf{P}\right] \mathbf{I}$ in the area indicated in (a). (c) CLSM images of micelles of [PT $\left.\mathbf{P}_{\mathbf{3 0}} \mathbf{P}\right] \mathbf{I}$ detected at each wavelength. 


\section{Self-assembly of $[\mathbf{P T} \underline{\mathbf{5 0}} \underline{\mathbf{P}] \mathbf{I}}$}

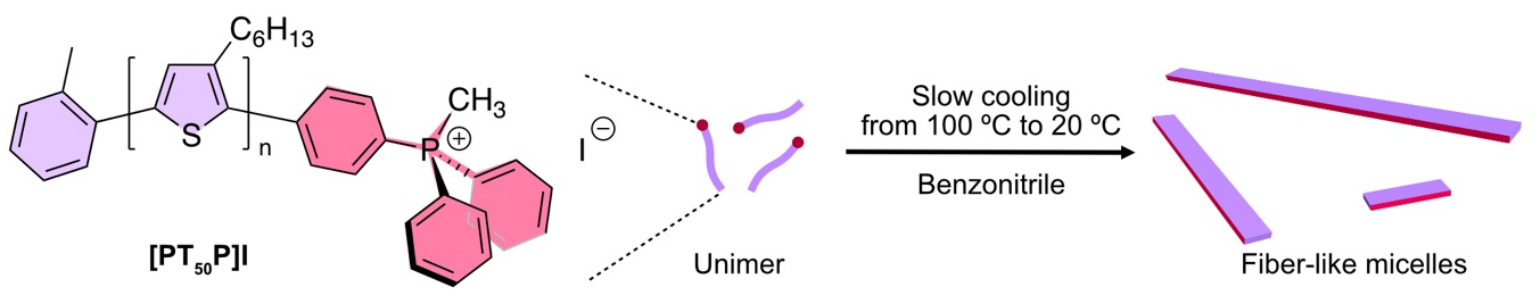

b

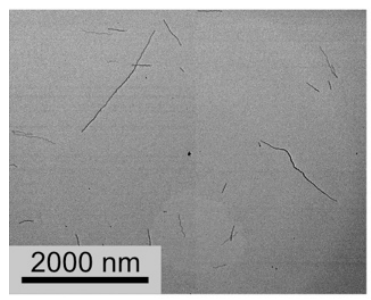

c

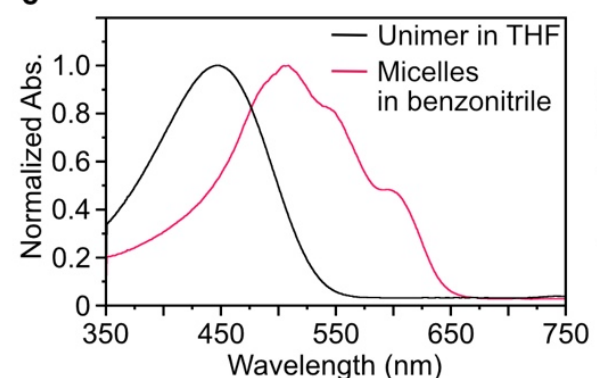

d

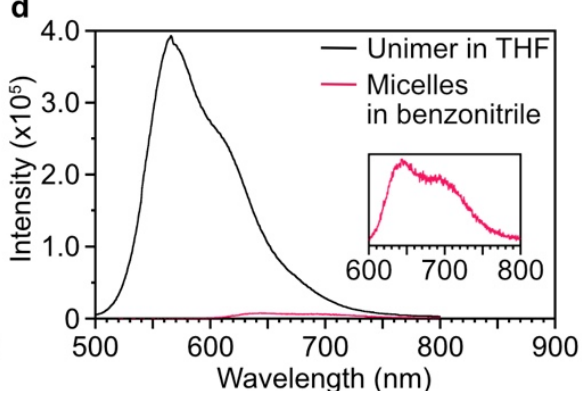

Figure S8.

(a) Schematic representation of self-assembly of $\left[\mathbf{P T}_{\mathbf{5 0}} \mathbf{P}\right] \mathbf{I}$ in benzonitrile. (b) TEM image of the fiber-like micelles of $\left[\mathbf{P} \mathbf{T}_{50} \mathbf{P}\right] \mathbf{I}$ on carbon-coated $\mathrm{Cu}$ grid. (c) Absorption spectra of $\left[\mathbf{P T} \mathbf{T}_{\mathbf{5 0}} \mathbf{P}\right] \mathbf{I}$ in THF (black) or benzonitrile (red) (Conc. $=0.02 \mathrm{mg} / \mathrm{mL})$. (d) Photoluminescence spectra of $\left[\mathbf{P T}_{50} \mathbf{P}\right] \mathbf{I}$ in THF (black) or benzonitrile (red) (Conc.: $0.02 \mathrm{mg} / \mathrm{mL}, \lambda_{\mathrm{ex}}=440 \mathrm{~nm}$ for THF solution, $\lambda_{\mathrm{ex}}=513 \mathrm{~nm}$ for benzonitrile solution). 
a

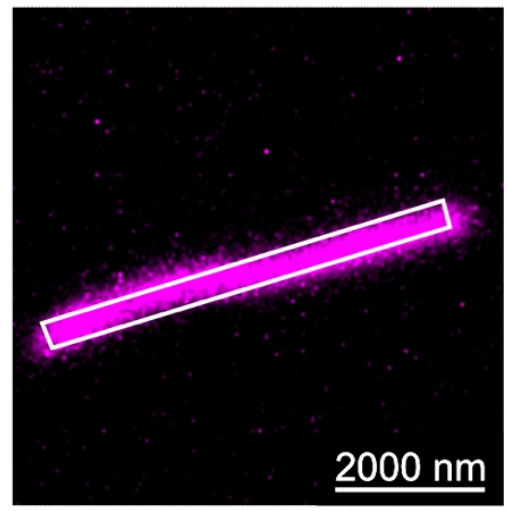

b

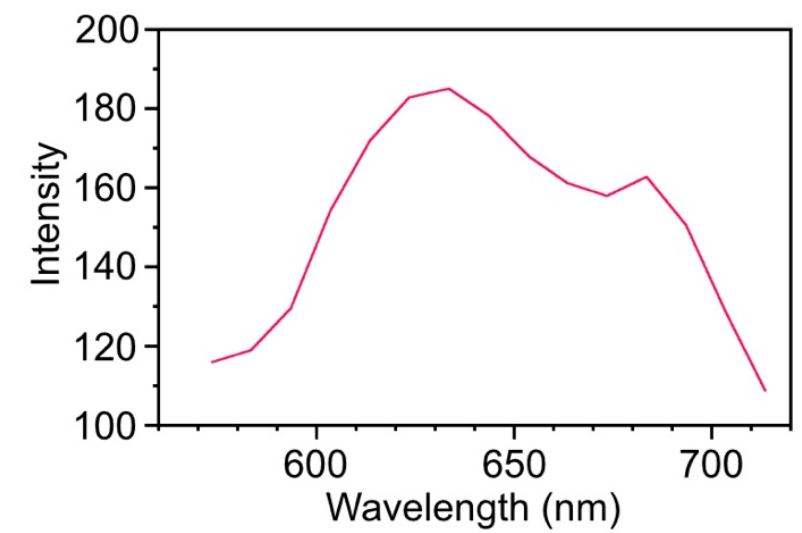

C

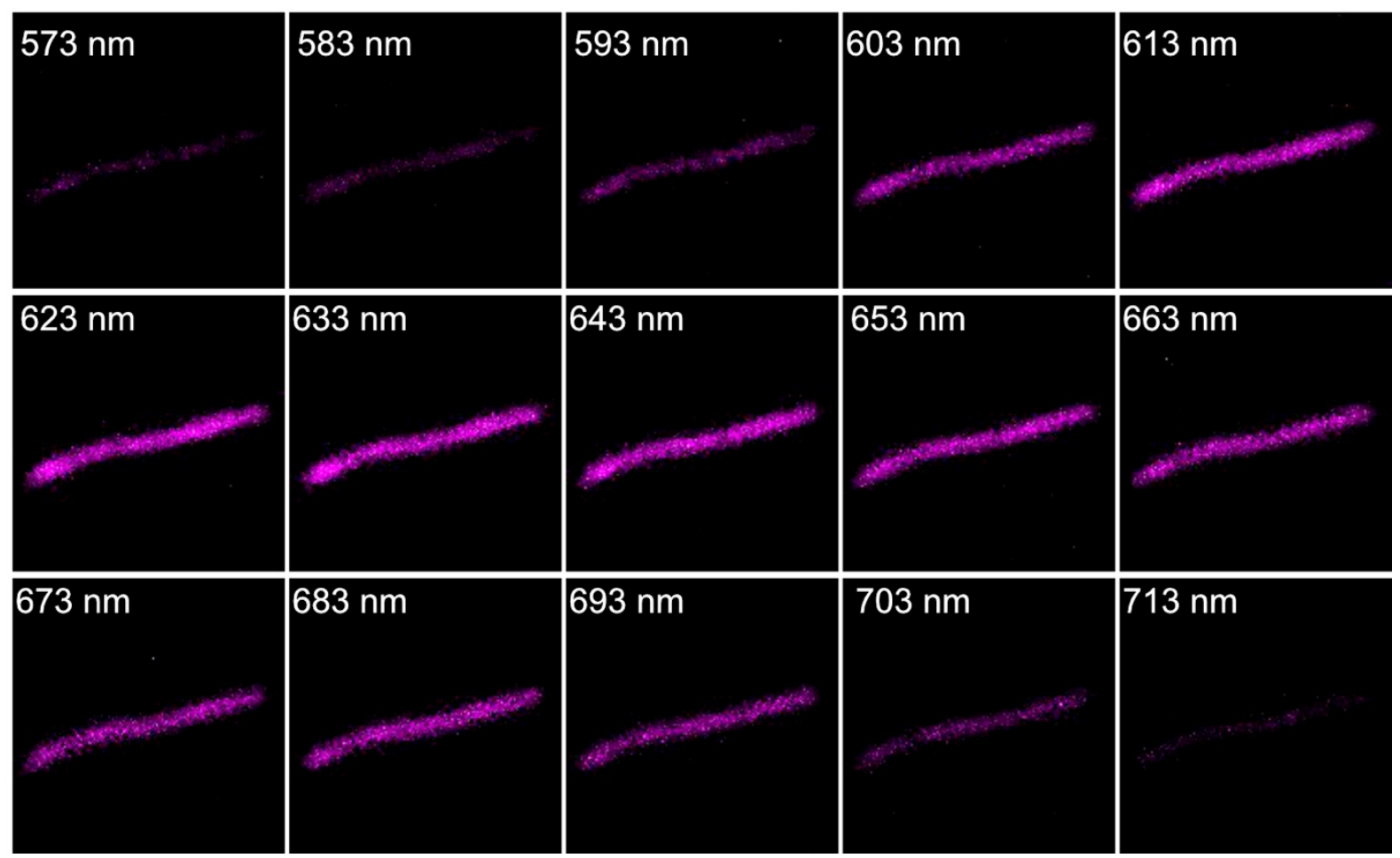

Figure S9.

(a) CLSM image of the fiber-like micelles of $\left[\mathbf{P T}_{\mathbf{5 0}} \mathbf{P}\right] \mathbf{I}$ in benzonitrile $($ Conc. $=0.02 \mathrm{mg} / \mathrm{mL})$. (b) Photoluminescence spectrum of a single fiber-like micelle of $\left[\mathbf{P T}_{\mathbf{5 0}} \mathbf{P}\right] \mathbf{I}$ in the area indicated in (a). (c) CLSM images of micelles of [PT $\left.{ }_{50} \mathbf{P}\right] \mathbf{I}$ detected at each wavelength. 
Width of fiber-like micelles derived from $\left[\mathbf{P} \mathbf{T}_{\underline{n}} \mathbf{P}\right] \mathbf{I}(\mathrm{n}=30$ and 50$)$

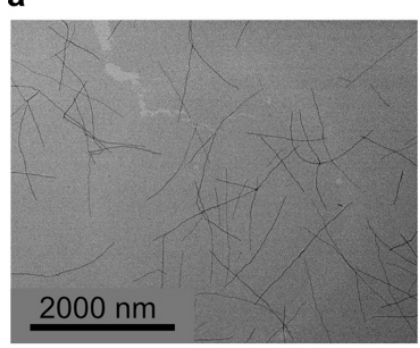

d

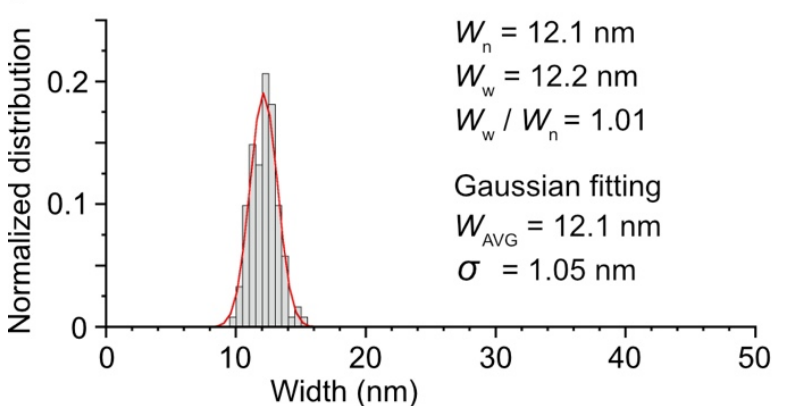

b

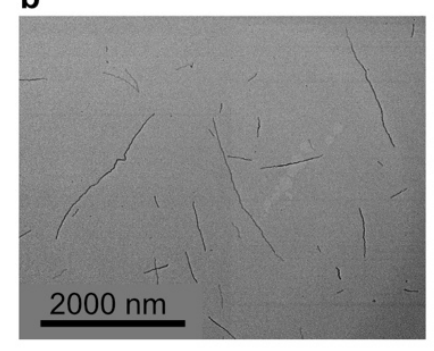

C

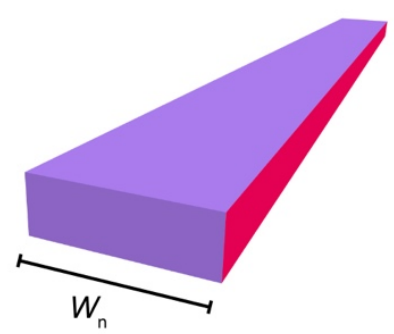

e

\section{Figure S10.}

(a and b) TEM images of the fiber-like micelles derived from $\left[\mathbf{P T} \mathbf{T}_{\mathbf{n}} \mathbf{P}\right] \mathbf{I} ;(\mathbf{a}) \mathrm{n}=30,(\mathbf{b})=50$. (c) Schematic representation of the width of fiber-like micelles. (d) Histogram of the width of fiber -like micelles of $\left[\mathbf{P T}_{\mathbf{3 0}} \mathbf{P}\right] \mathbf{I}$. (e) Histogram of the width of fiber -like micelles of $\left[\mathbf{P T} \mathbf{T}_{\mathbf{5 0}} \mathbf{P}\right] \mathbf{I}$. Gaussian fitting was performed by Eq. 4 .

$$
f(x)=a \cdot \exp \left\{-\frac{\left(x-W_{A V G}\right)^{2}}{2 \sigma^{2}}\right\}
$$

The extended chain length of the PT backbone was calculated based on the unit cell in Table $\mathrm{S} 1$. The $c$-axis distance represents the length of a bithiophene unit $(0.76 \mathrm{~nm})$. Therefore, the width of a $\left[\mathbf{P T}_{\mathbf{3 0}} \mathbf{P}\right] \mathbf{I}$ fiber-like micelle can be calculated as $11.4 \mathrm{~nm}(=0.76 \times 30 / 2 \mathrm{~nm})$. Similarly, the width for $\left[\mathbf{P T}_{\mathbf{5 0}} \mathbf{P}\right] \mathbf{I}$ can be calculated as $19 \mathrm{~nm}(=0.76 \times 50 / 2 \mathrm{~nm})$. 
$\underline{\text { XRD for the film of fiber-like micelles derived from }[\mathbf{P T}} \underline{\mathbf{P}} \underline{\mathbf{P}] \mathbf{I}}$

a

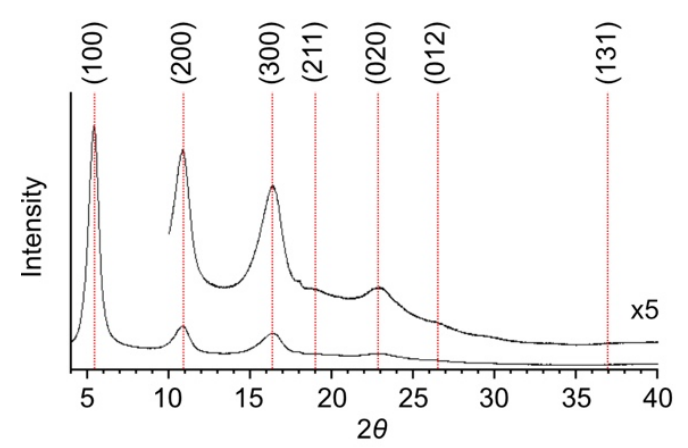

b

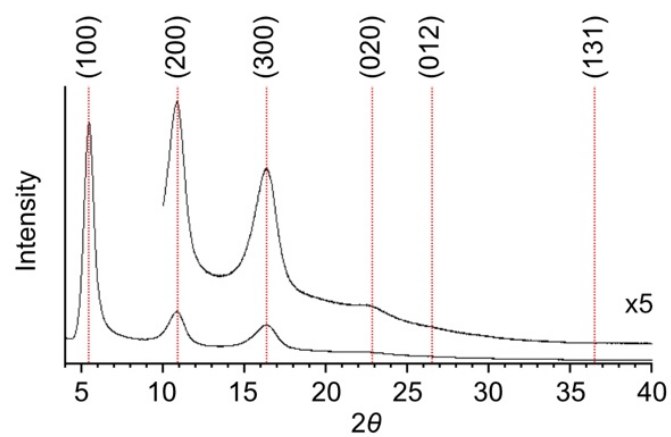

c

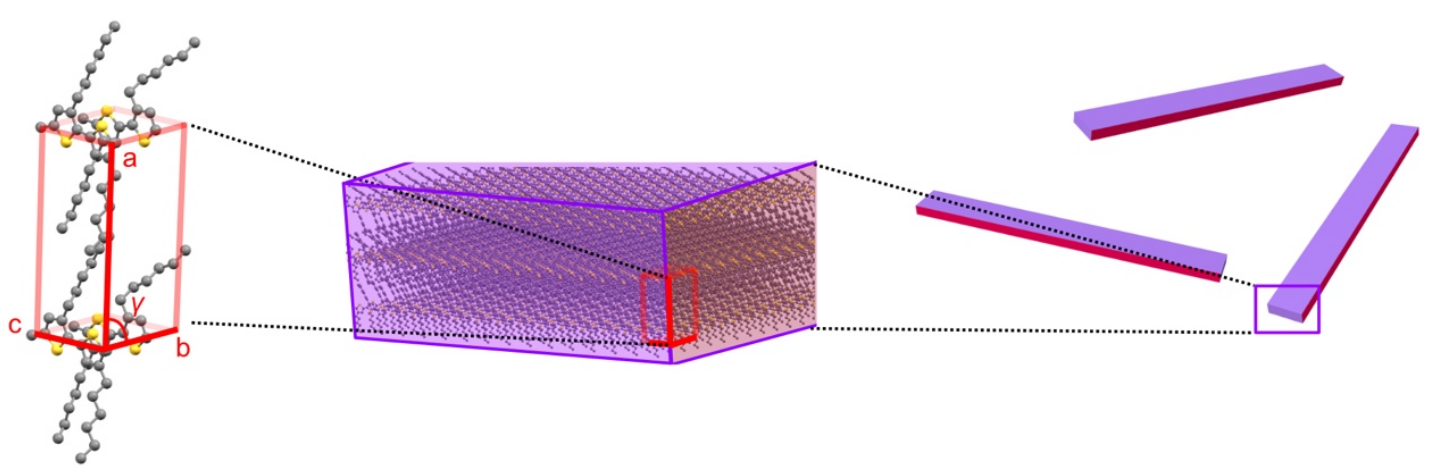

Figure S11.

(a and b) Powder XRD for the films of the fiber-like micelles: $\left[\mathbf{P T}_{\mathbf{3 0}} \mathbf{P}\right] \mathbf{I}(\mathbf{a})$ and $\left[\mathbf{P T}_{\mathbf{5 0}} \mathbf{P}\right] \mathbf{I}(\mathbf{b})$. (c) Schematic representation of the unit cell of the fiber-like micelles. The diffraction pattern was almost identical to that in the literature ${ }^{\mathrm{S} 2}$.

$\underline{\text { Table S1: The unit cell of fiber-like micelles derived from }[\mathbf{P T}} \underline{\mathbf{n}} \underline{\mathbf{P}] \mathbf{I}(\mathrm{n}=30,50)}{ }^{\mathrm{S} 2}$.

\begin{tabular}{|c|c|c|} 
& \multicolumn{1}{|c|}{$\left[\mathbf{P T}_{\mathbf{3 0}} \mathbf{P}\right] \mathbf{I}$} & {$\left[\mathbf{P T}_{\mathbf{5 0}} \mathbf{P}\right] \mathbf{I}$} \\
\hline $\begin{array}{c}\text { Crystal } \\
\text { system }\end{array}$ & Monoclinic & Monoclinic \\
\hline $\mathbf{a}(\AA)$ & 16.3 & 16.3 \\
\hline $\mathbf{b}(\AA)$ & 7.7 & 7.7 \\
\hline $\mathbf{c}(\AA)$ & 7.6 & 7.6 \\
\hline$\gamma\left(\mathbf{(}^{\mathbf{(}}\right)$ & 83 & 89 \\
\hline
\end{tabular}




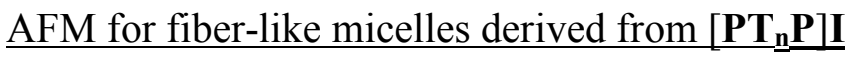
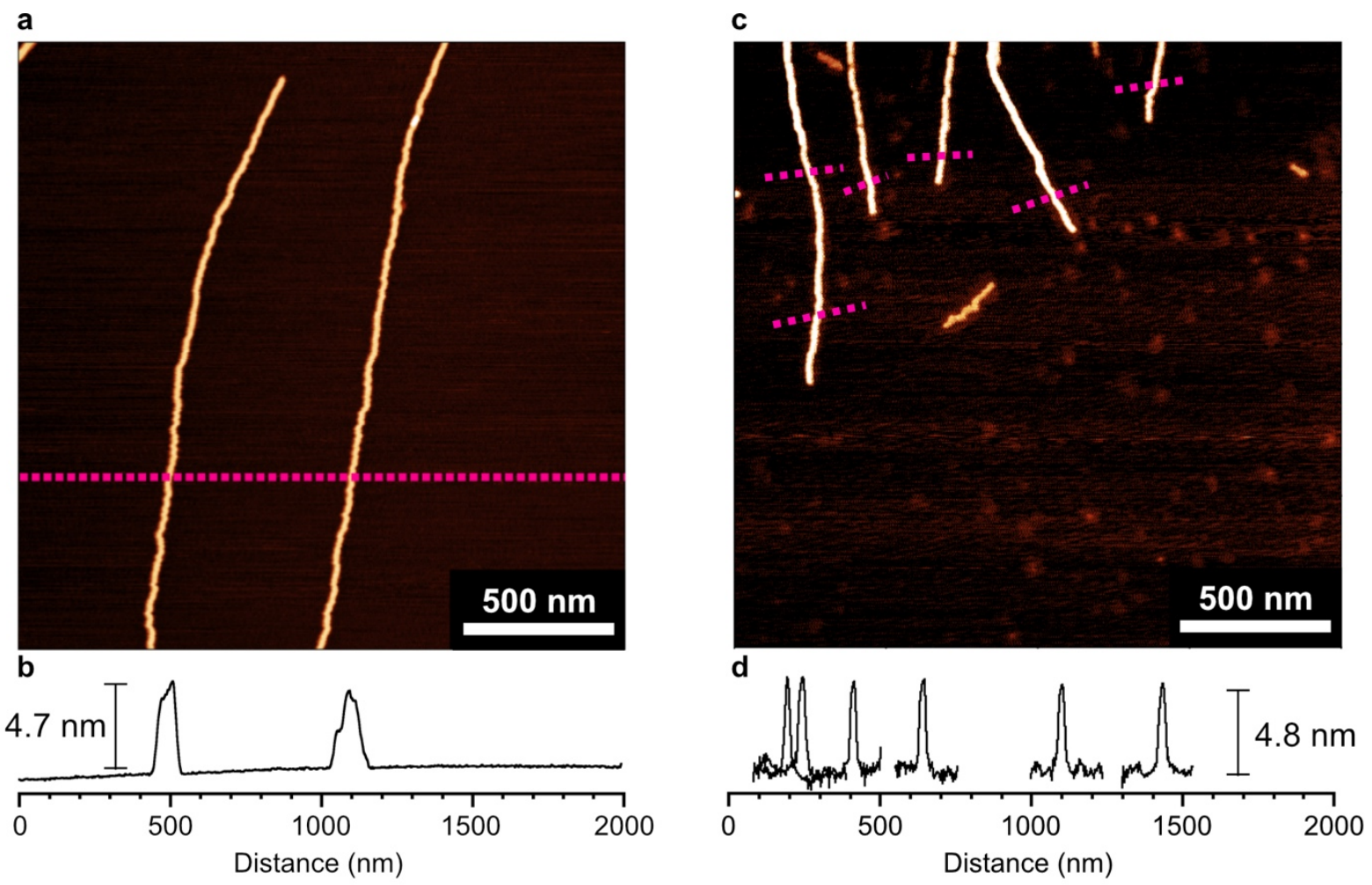

Figure S12.

(a and b) AFM images (a) and the height profiles (b) of fiber-like micelles derived from $\left[\mathbf{P T}_{\mathbf{3 0}} \mathbf{P}\right] \mathbf{I}$. (c and $\left.\mathbf{d}\right)$ AFM images (c) and the height profiles (d) of fiber-like micelles derived from $\left[\mathbf{P T} \mathbf{5 0}_{\mathbf{5}} \mathbf{P}\right] \mathbf{I}$. 
Variable-temperature UV/vis absorption spectra of $\left[\mathbf{P T} \underline{\mathbf{1}_{1}} \underline{\mathbf{P}}\right] \mathbf{I}$ in solution In benzonitrile

a

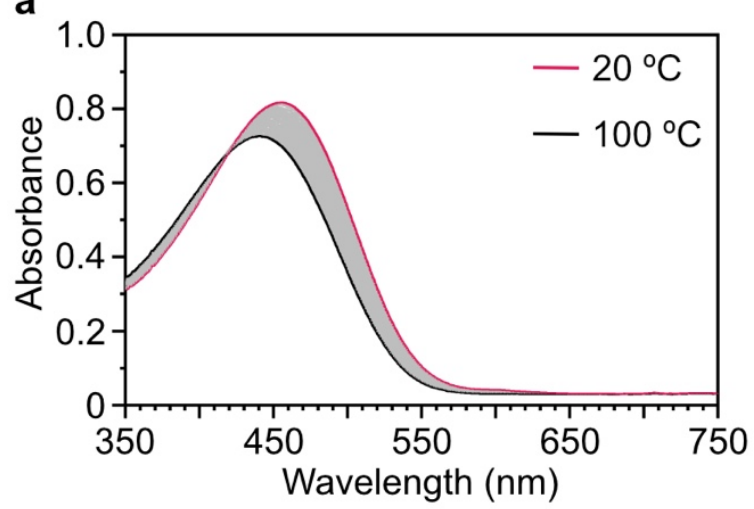

C

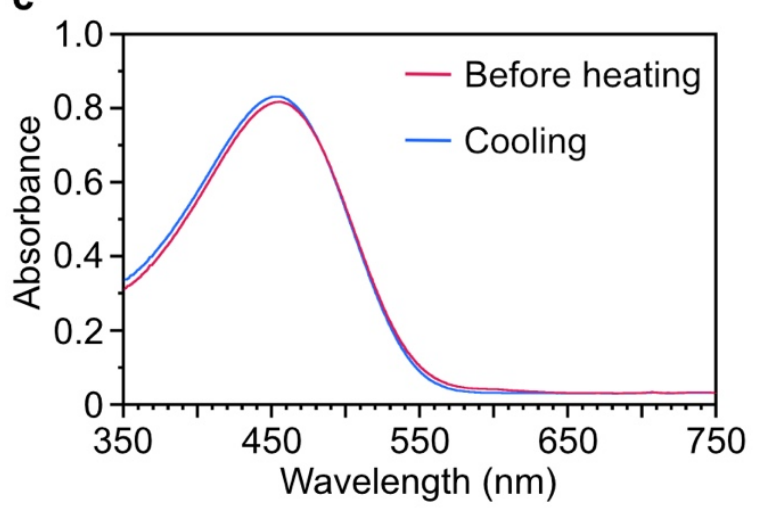

b
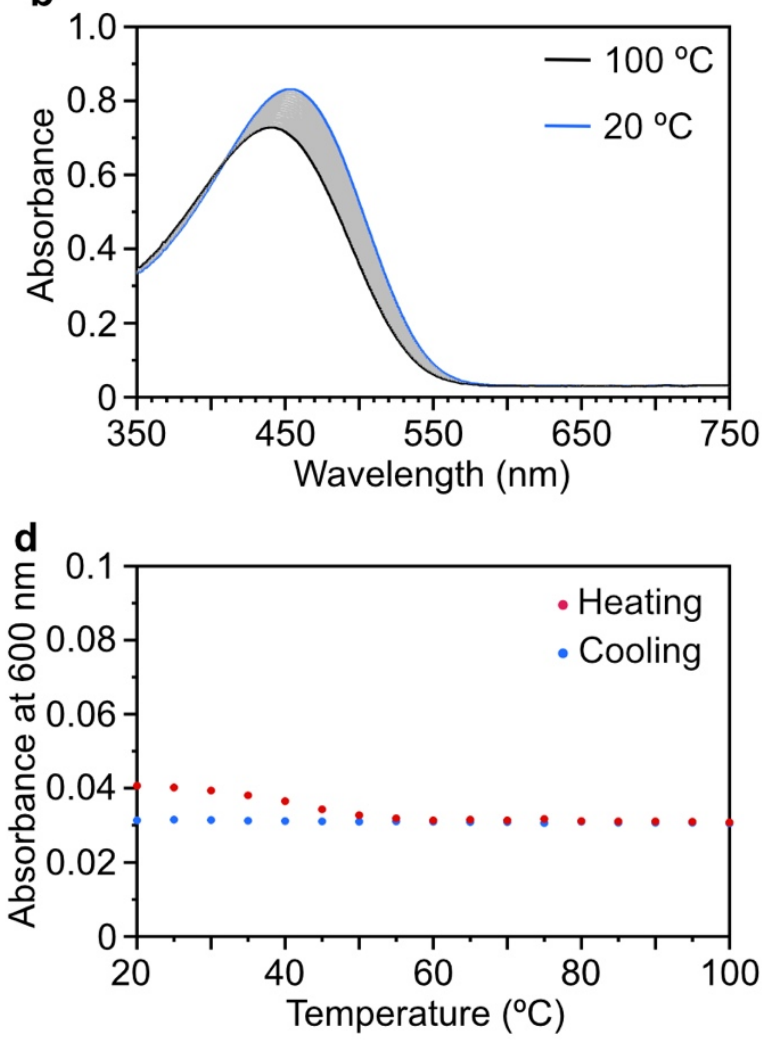

In THF

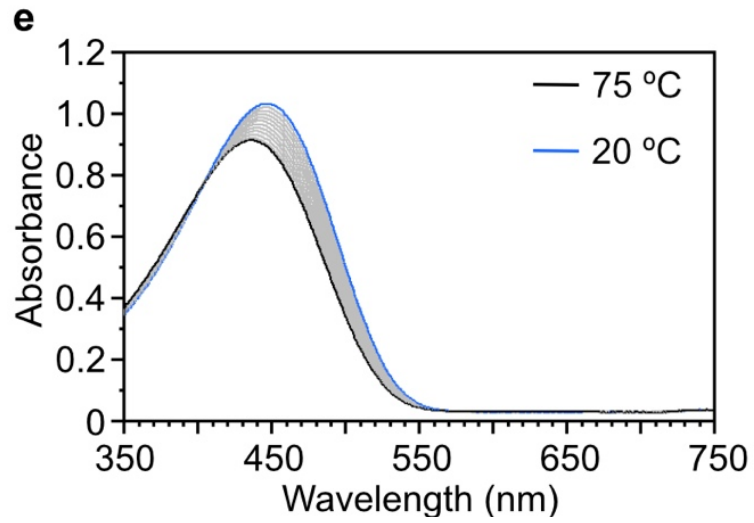

Figure S13.

(a) Temperature-dependent absorption spectra upon heating from $20{ }^{\circ} \mathrm{C}$ to $100{ }^{\circ} \mathrm{C}$ for the benzonitrile solution of $\left[\mathbf{P} \mathbf{T}_{19} \mathbf{P}\right] \mathbf{I}$ micelles, where each spectrum was recorded every $5{ }^{\circ} \mathrm{C}$ interval (Heating rate: $1^{\circ} \mathrm{C} / \mathrm{min}$, Conc. $=0.02 \mathrm{mg} / \mathrm{mL}$ ). (b) Temperature-dependent absorption spectra upon cooling from $100{ }^{\circ} \mathrm{C}$ to $20{ }^{\circ} \mathrm{C}$ for the benzonitrile solution of unimers $\left[\mathbf{P T}{ }_{19} \mathbf{P}\right] \mathbf{I}$ (Cooling rate: $1^{\circ} \mathrm{C} / \mathrm{min}$, Conc. $=0.02 \mathrm{mg} / \mathrm{mL}$ ). $(\mathbf{c})$ Absorption spectra of a solution of $\left[\mathbf{P T}{ }_{19} \mathbf{P}\right] \mathbf{I}$ in benzonitrile at $20^{\circ} \mathrm{C}$ before heating (pink) or after both heating and cooling processes (blue). (d) Temperature-dependent change in absorbance at $600 \mathrm{~nm}$ of $\left[\mathbf{P T}_{19} \mathbf{P}\right] \mathbf{I}$. (e) Temperaturedependent absorption spectra upon cooling from $75^{\circ} \mathrm{C}$ to $20^{\circ} \mathrm{C}$ for the THF solution of unimers $\left[\mathbf{P T}{ }_{\imath} \mathbf{P}\right] \mathbf{I}$ (Cooling rate: $1^{\circ} \mathrm{C} / \mathrm{min}$, Conc. $\left.=0.02 \mathrm{mg} / \mathrm{mL}\right)$. 
Variable-temperature UV/vis absorption spectra of $[\mathbf{P T} \underline{\mathbf{3 0}} \underline{\mathbf{P}}] \mathbf{I}$ in solution

In benzonitrile

a

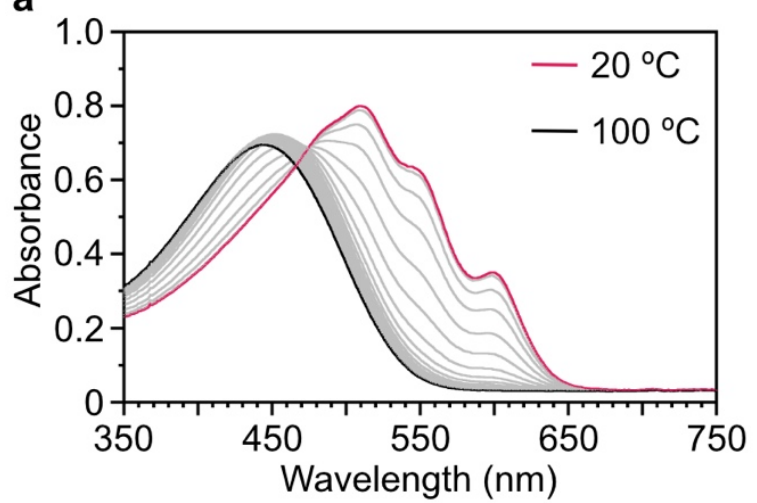

C

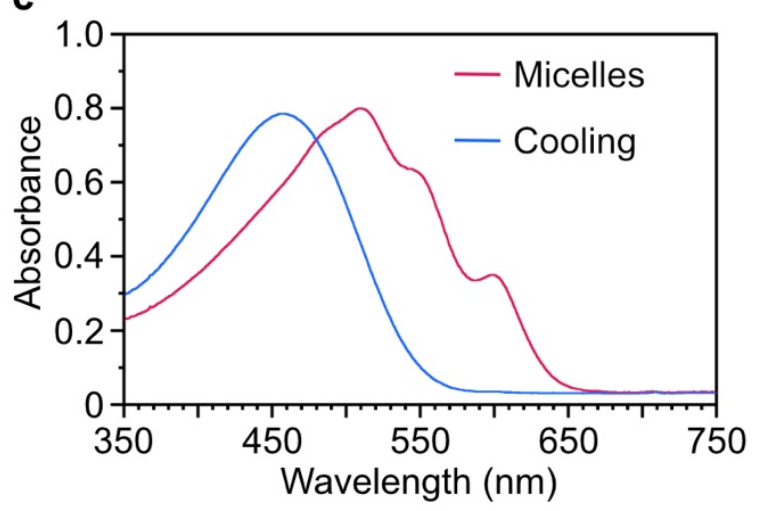

b
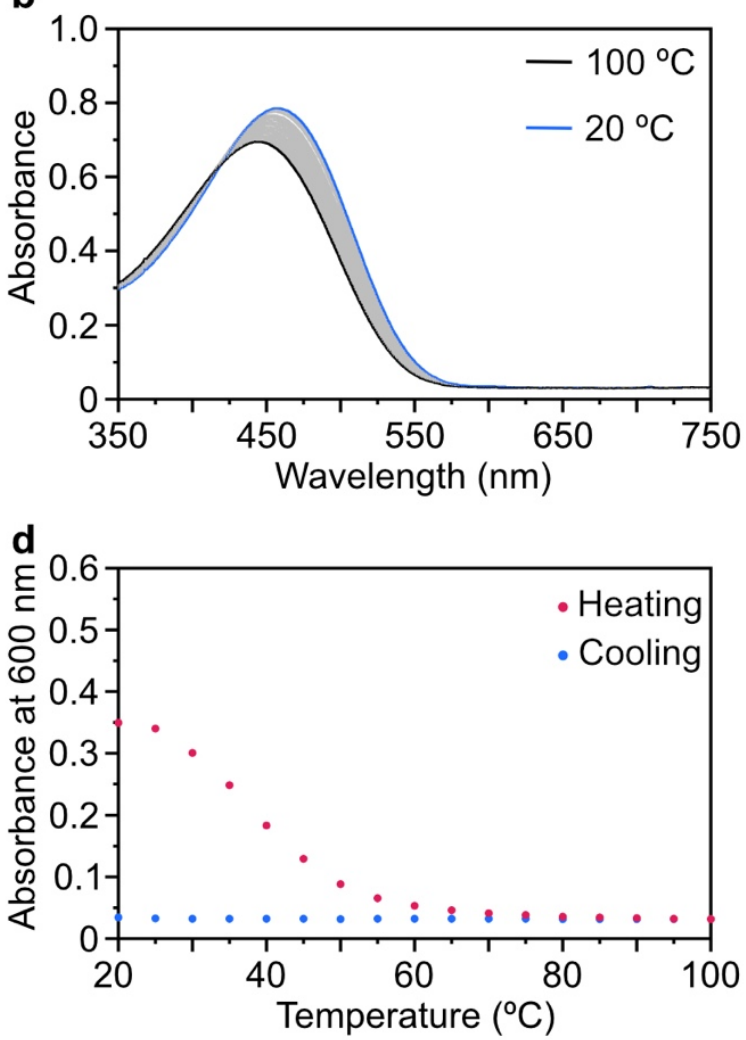

In THF

e

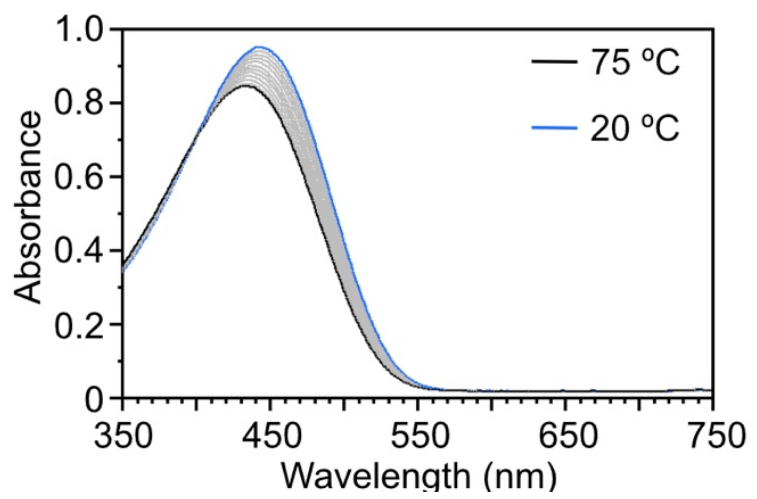

Figure S14.

(a) Temperature-dependent absorption spectra upon heating from $20{ }^{\circ} \mathrm{C}$ to $100{ }^{\circ} \mathrm{C}$ for the benzonitrile solution of micelles $\left[\mathbf{P} \mathbf{T}_{30} \mathbf{P}\right] \mathbf{I}$, where each spectrum was recorded every $5{ }^{\circ} \mathrm{C}$ interval (Heating rate: $1{ }^{\circ} \mathrm{C} / \mathrm{min}$, Conc. $=0.02 \mathrm{mg} / \mathrm{mL}$ ). (b) Temperature-dependent absorption spectra upon cooling from $100{ }^{\circ} \mathrm{C}$ to $20{ }^{\circ} \mathrm{C}$ for the benzonitrile solution of unimers $\left[\mathbf{P T}_{30} \mathbf{P}\right] \mathbf{I}$ (Cooling rate: $1{ }^{\circ} \mathrm{C} / \mathrm{min}$, Conc. $=0.02 \mathrm{mg} / \mathrm{mL}$ ). $(\mathbf{c})$ Absorption spectra of a solution of $\left[\mathbf{P T} \mathbf{T}_{30} \mathbf{P}\right] \mathbf{I}$ in benzonitrile at $20^{\circ} \mathrm{C}$ before heating (pink) or after both heating and cooling processes (blue). (d) Temperature-dependent change in absorbance at $600 \mathrm{~nm}$ of $\left[\mathbf{P T}_{30} \mathbf{P}\right] \mathbf{I}$. (e) Temperaturedependent absorption spectra upon cooling from $75^{\circ} \mathrm{C}$ to $20^{\circ} \mathrm{C}$ for the THF solution of unimers $\left[\right.$ PT $\left._{30} \mathbf{P}\right] \mathbf{I}\left(\right.$ Cooling rate: $1{ }^{\circ} \mathrm{C} / \mathrm{min}$, Conc. $\left.=0.02 \mathrm{mg} / \mathrm{mL}\right)$. 
Variable-temperature UV/vis absorption spectra of $\left[\mathbf{P T} \underline{\mathbf{5}_{\mathbf{0}}} \underline{\mathbf{P}}\right] \mathbf{I}$ in solution

In benzonitrile

a

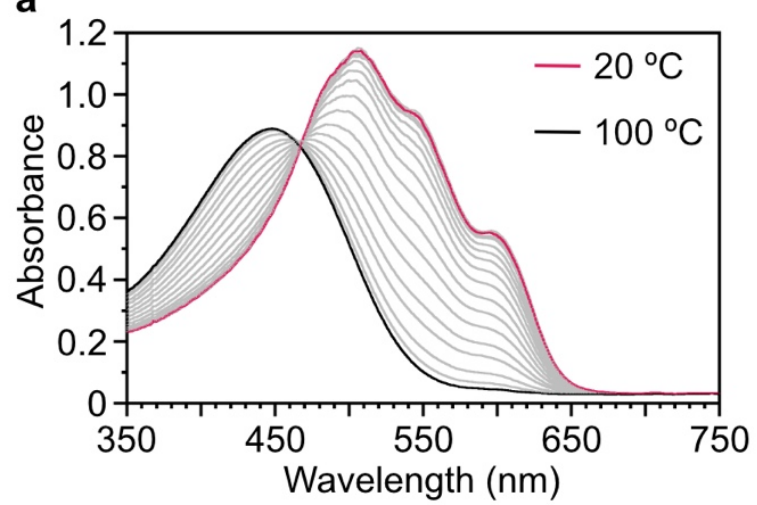

C

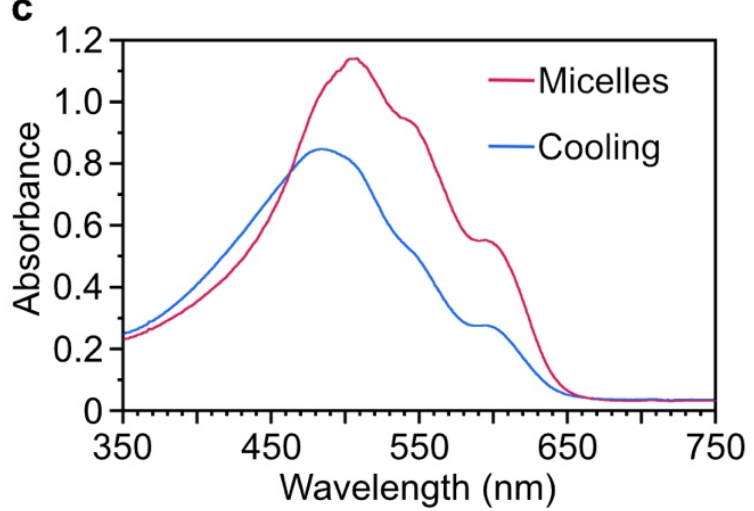

b
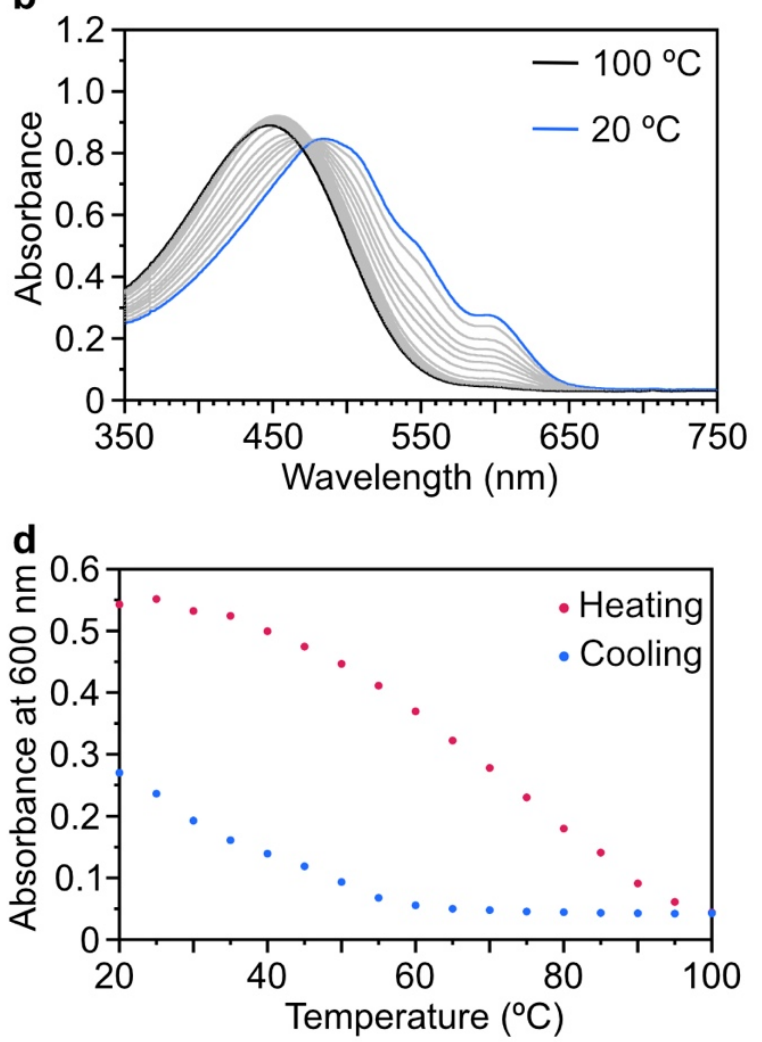

In THF

e

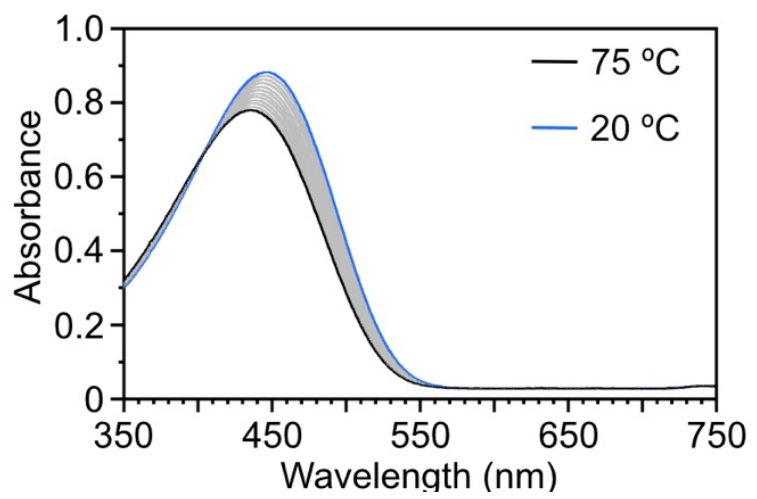

Figure S15.

(a) Temperature-dependent absorption spectra upon heating from $20{ }^{\circ} \mathrm{C}$ to $100{ }^{\circ} \mathrm{C}$ for the benzonitrile solution of micelles $\left[\mathbf{P} \mathbf{T}_{s_{0}} \mathbf{P}\right] \mathbf{I}$, where each spectrum was recorded every $5{ }^{\circ} \mathrm{C}$ interval (Heating rate: $1{ }^{\circ} \mathrm{C} / \mathrm{min}$, Conc. $=0.02 \mathrm{mg} / \mathrm{mL}$ ). (b) Temperature-dependent absorption spectra upon cooling from $100{ }^{\circ} \mathrm{C}$ to $20{ }^{\circ} \mathrm{C}$ for the benzonitrile solution of unimers $\left[\mathbf{P T}{ }_{50} \mathbf{P}\right] \mathbf{I}$ (Cooling rate: $1{ }^{\circ} \mathrm{C} / \mathrm{min}$, Conc. $\left.=0.02 \mathrm{mg} / \mathrm{mL}\right)$. $(\mathbf{c})$ Absorption spectra of a solution of $\left[\mathbf{P} \mathbf{T}_{\mathrm{s} 0} \mathbf{P}\right] \mathbf{I}$ in benzonitrile at $20^{\circ} \mathrm{C}$ before heating (pink) or after both heating and cooling processes (blue). (d) Temperature-dependent change in absorbance at $600 \mathrm{~nm}$ of $\left[\mathbf{P T}_{50} \mathbf{P}\right] \mathbf{I}$. (e) Temperaturedependent absorption spectra upon cooling from $75^{\circ} \mathrm{C}$ to $20^{\circ} \mathrm{C}$ for the THF solution of unimers $\left[\mathbf{P T}_{\mathrm{s} 0} \mathbf{P}\right] \mathbf{I}$ (Cooling rate: $1^{\circ} \mathrm{C} / \mathrm{min}$, Conc. $\left.=0.02 \mathrm{mg} / \mathrm{mL}\right)$. 
Monitoring the self-assembly kinetics: evaluation of the self-nucleation process.

a

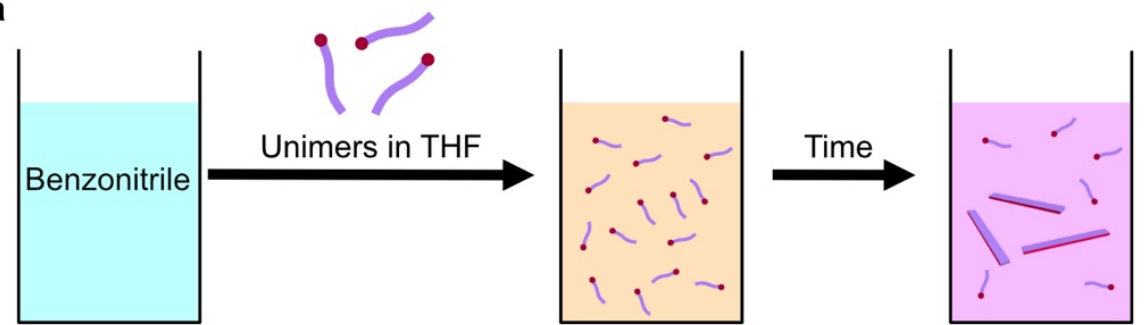

b

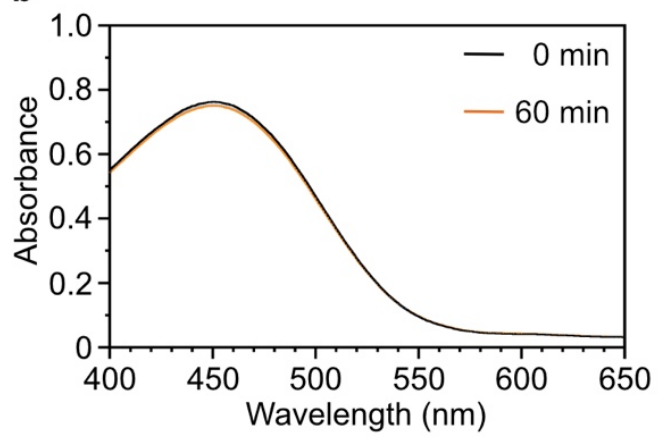

d

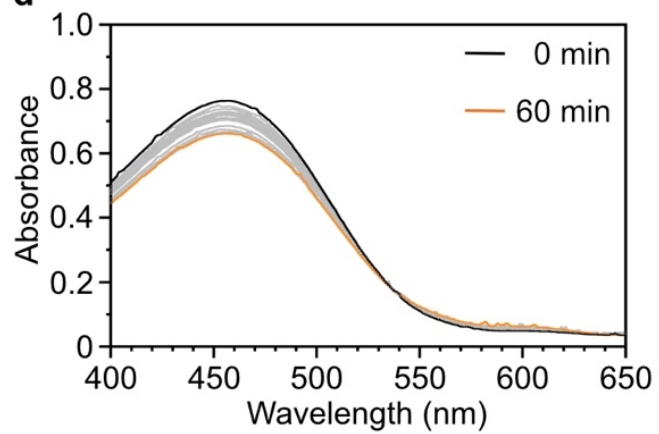

f

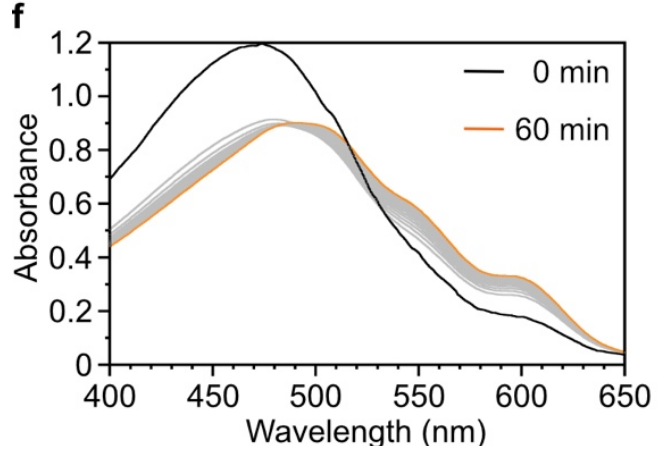

c

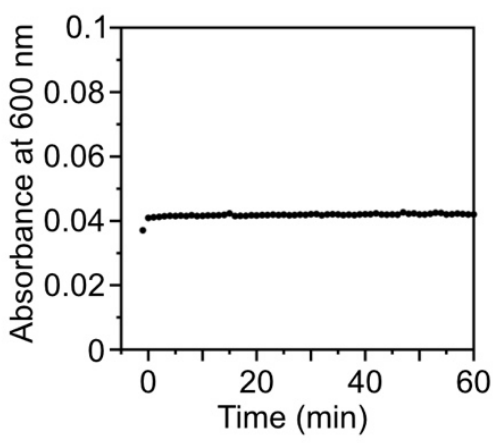

e
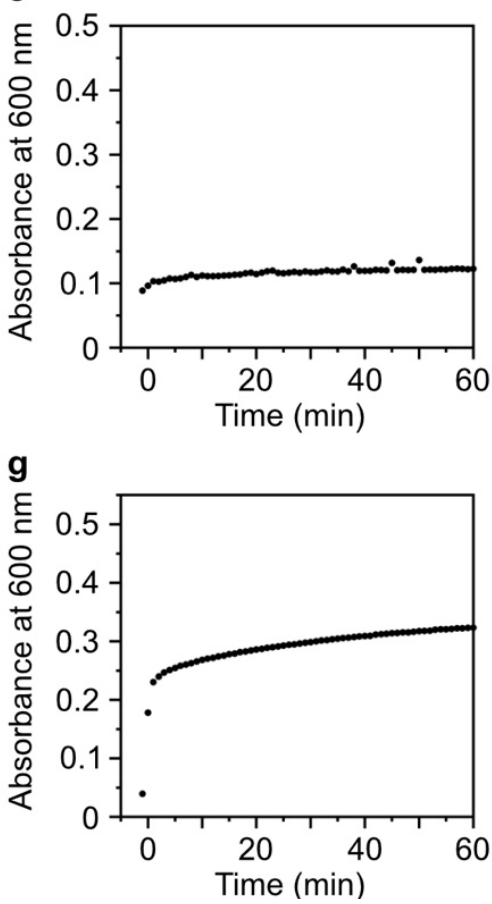

Figure S16.

(a) Schematic representation of the injection of unimers (Conc. $=1.0 \mathrm{mg} / \mathrm{mL}$ in THF) into pure benzonitrile at $35^{\circ} \mathrm{C}$ to evaluate the self-nucleation which was monitored by UV/vis absorption spectroscopy in $1 \mathrm{~min}$ interval (final Conc.$=0.02 \mathrm{mg} / \mathrm{mL}$ ). $(\mathbf{b}, \mathbf{d}, \mathbf{f})$ Time-dependent absorption spectral changes after the addition of the unimers of $\left[\mathbf{P T} \mathbf{T}_{\mathbf{n}} \mathbf{P}\right] \mathbf{I}$ to benzonitrile: $\mathrm{n}=19(\mathbf{b}), 30(\mathbf{d})$, and 50 (f). (c, e, g) Time-dependent change in absorbance at $600 \mathrm{~nm}$ of $\left[\mathbf{P T} \mathbf{n}_{n} \mathbf{P}\right] \mathbf{I}$ after the injection of unimers: $\mathrm{n}=19(\mathbf{c}), 30(\mathbf{e})$, and $50(\mathbf{g})$. 
Energy landscape of the self-assembly for $[\mathbf{P T} \underline{\mathbf{P}} \underline{\mathbf{P}}] \mathbf{I}$.

a in $\mathrm{THF}$

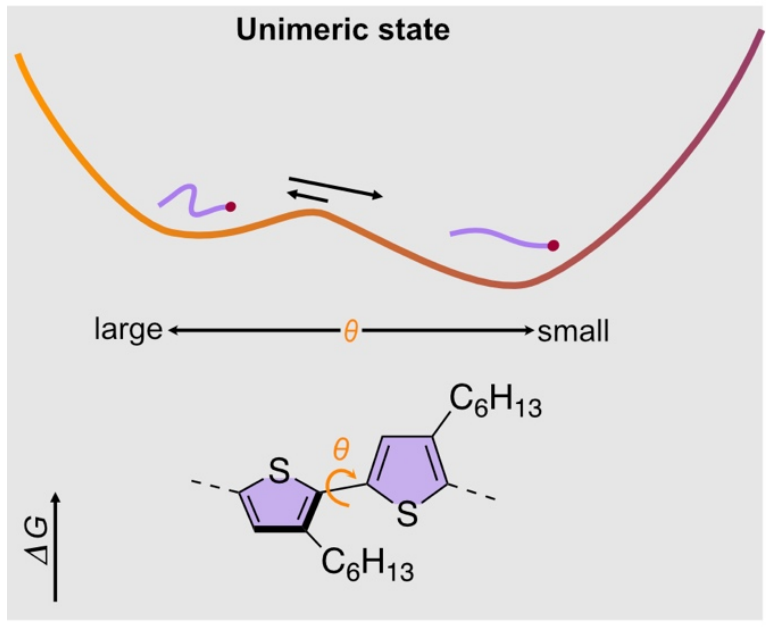

High $=$ Temperature $\longrightarrow$ Low

b in benzonitrile

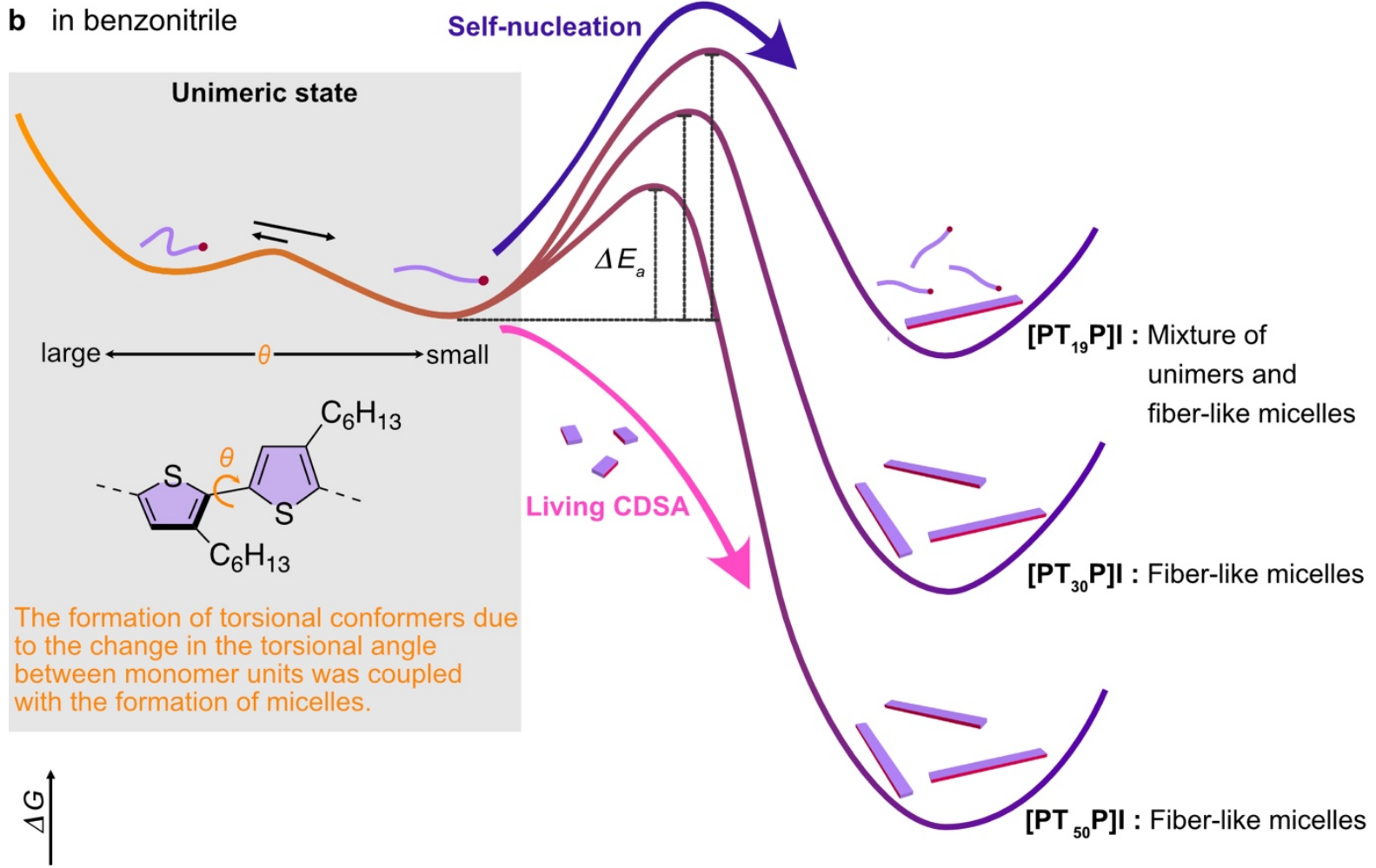

High

Temperature

Low

Figure S17.

Free energy landscape of the self-assembly of $[\mathbf{P T}$ P]I in (a) THF or (b) benzonitrile. Crystallization-driven self-assembly (CDSA) should overcome the activation barrier $\left(\Delta E_{\mathrm{a}}\right)$ for the nucleation to form fiber-like micelles, whereas living CDSA can bypass the nucleation process because the seeds act as 'nuclei'. 


\section{Seed micelles of $\left[\mathbf{P T}_{\underline{\mathbf{3}} \underline{\mathbf{P}}} \underline{\mathbf{P}] \text { and }[\mathbf{P T}} \underline{\mathbf{5 0}} \underline{\mathbf{P}] \mathbf{I}}\right.$}

a

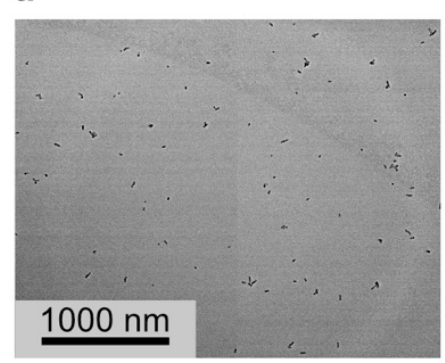

b

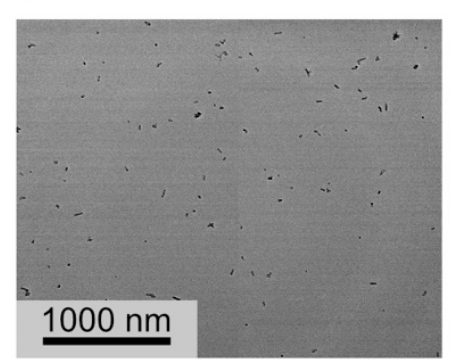

$1000 \mathrm{~nm}$

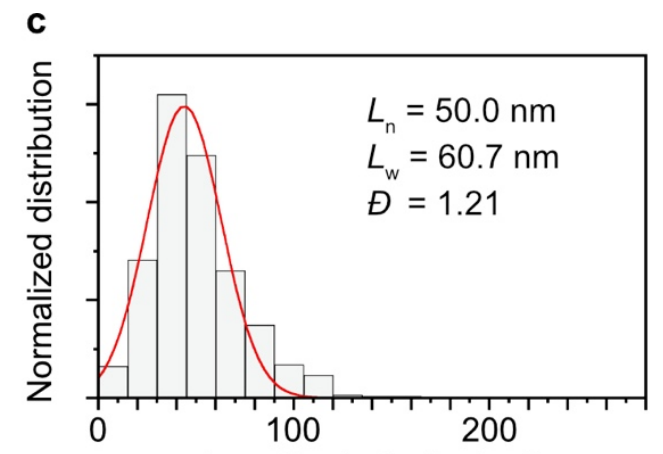

Length of micelle $(\mathrm{nm})$

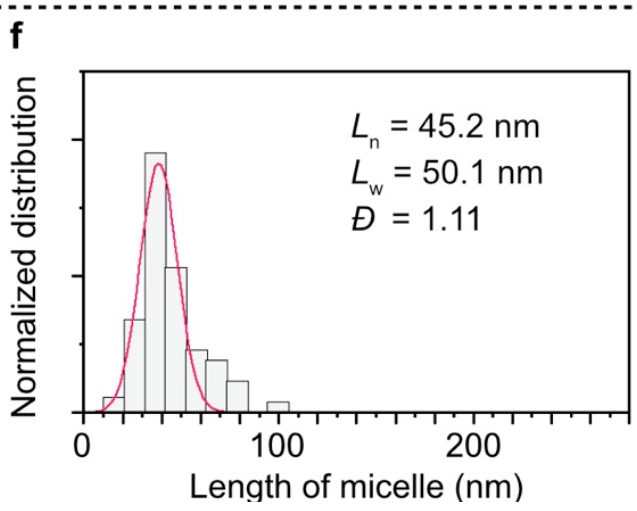

\section{Figure S18.}

(a and b) TEM images of seed micelles consist of $\left[\mathbf{P T} \mathbf{T}_{\mathbf{3 0}} \mathbf{P}\right] \mathbf{I}$ in benzonitrile after the sonication and the thermal annealing. (c) Histogram of the length of seed micelles. Red line is the result of the Gaussian fitting (Eq. 4). (d and e) TEM images of seed micelles consist of [PT $\left.\mathbf{P}_{\mathbf{5 0}} \mathbf{P}\right] \mathbf{I}$ in benzonitrile after the sonication and the thermal annealing. (f) Histogram of the length of seed micelles. The red line is the result of the Gaussian fitting (Eq. 4).

$$
f(x)=a \cdot \exp \left\{-\frac{\left(x-L_{A V G}\right)^{2}}{2 \sigma^{2}}\right\}
$$


$\underline{\text { Table S2: TEM analysis for the living CDSA of }[\mathbf{P T}} \underline{\mathbf{3 0}} \underline{\mathbf{P}] \mathbf{I}}$.

\begin{tabular}{|c|c|c|c|}
\hline $\boldsymbol{m}_{\text {unimer }} \boldsymbol{m}_{\text {seed }}$ & $\boldsymbol{L}_{\mathbf{n}}(\mathbf{n m})$ & $\boldsymbol{L}_{\mathbf{w}}(\mathbf{n m})$ & $\boldsymbol{\theta}$ \\
\hline $\mathbf{0}$ & 50.0 & 60.7 & 1.21 \\
\hline $\mathbf{5}$ & 283 & 309 & 1.09 \\
\hline $\mathbf{1 0}$ & 465 & 517 & 1.11 \\
\hline $\mathbf{2 0}$ & 971 & 1054 & 1.09 \\
\hline $\mathbf{4 0}$ & 1701 & 1812 & 1.07 \\
\hline
\end{tabular}


$\underline{\text { Length distribution of the resulting fiber-like micelles derived from }[\mathbf{P T}} \underline{\mathbf{3 0}} \underline{\mathbf{P}] \mathbf{I}}$

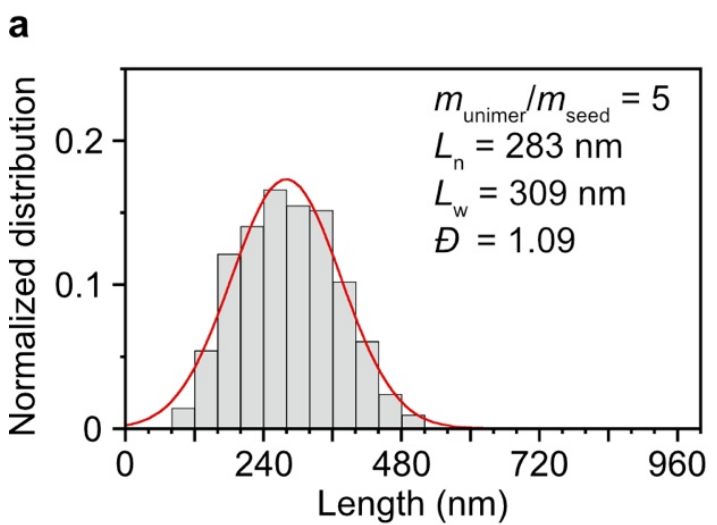

C

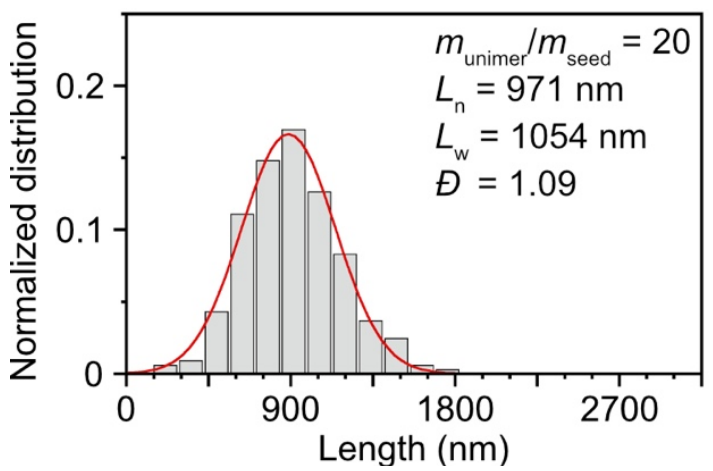

b

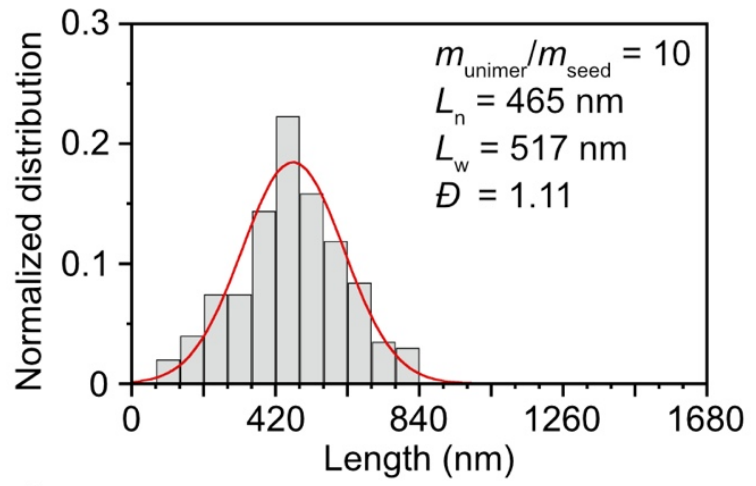

d

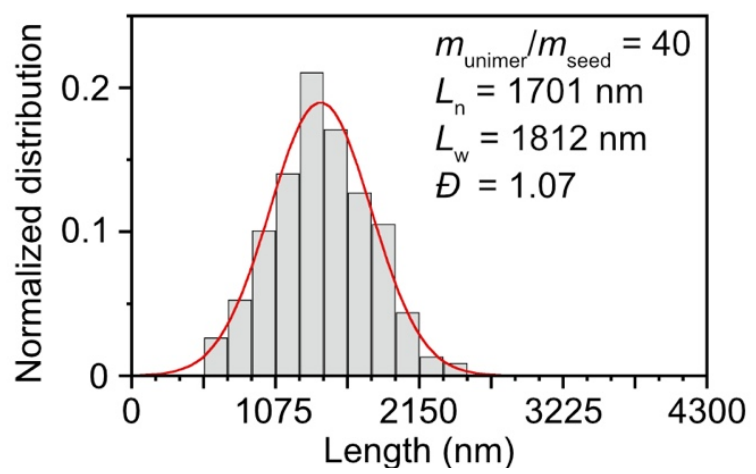

Figure S19.

Histogram of the length of resulting fiber-like micelles formed by living CDSA (numberaverage length of seed micelles was $50 \mathrm{~nm}$ as shown in Figure S18): unimer to seed ratio $\left(m_{\text {unimer }} / m_{\text {seed }}\right)=$ (a) 5 , (b) 10 , (c) 20 , and (d) 40 . Red lines were the results of the fitting by Gaussian function (Eq. 4).

$$
f(x)=\operatorname{aexp}\left\{-\frac{\left(x-L_{A V G}\right)^{2}}{2 \sigma^{2}}\right\}
$$


Table S3: The statistical analysis on the histogram of $[\mathbf{P T} \underline{\mathbf{3 0}} \underline{\mathbf{P}}] \mathbf{I}$.

\begin{tabular}{|c|c|c|c|}
\hline $\boldsymbol{m}_{\text {unimer }} \boldsymbol{m}_{\text {seed }}$ & $\boldsymbol{R}^{\mathbf{2}}$ & $\boldsymbol{L}_{\text {avg }}(\mathbf{n m})$ & $\boldsymbol{\sigma}(\mathbf{n m})$ \\
\hline $\mathbf{0}$ & 0.962 & 44.1 & 19 \\
\hline $\mathbf{5}$ & 0.983 & 280 & 95 \\
\hline $\mathbf{1 0}$ & 0.939 & 471 & 148 \\
\hline $\mathbf{2 0}$ & 0.990 & 948 & 274 \\
\hline $\mathbf{4 0}$ & 0.983 & 1695 & 455 \\
\hline
\end{tabular}


Living CDSA for $\left[\mathbf{P T}_{\underline{\mathbf{5 0}}} \mathbf{P}\right] \mathbf{I}$ in benzonitrile

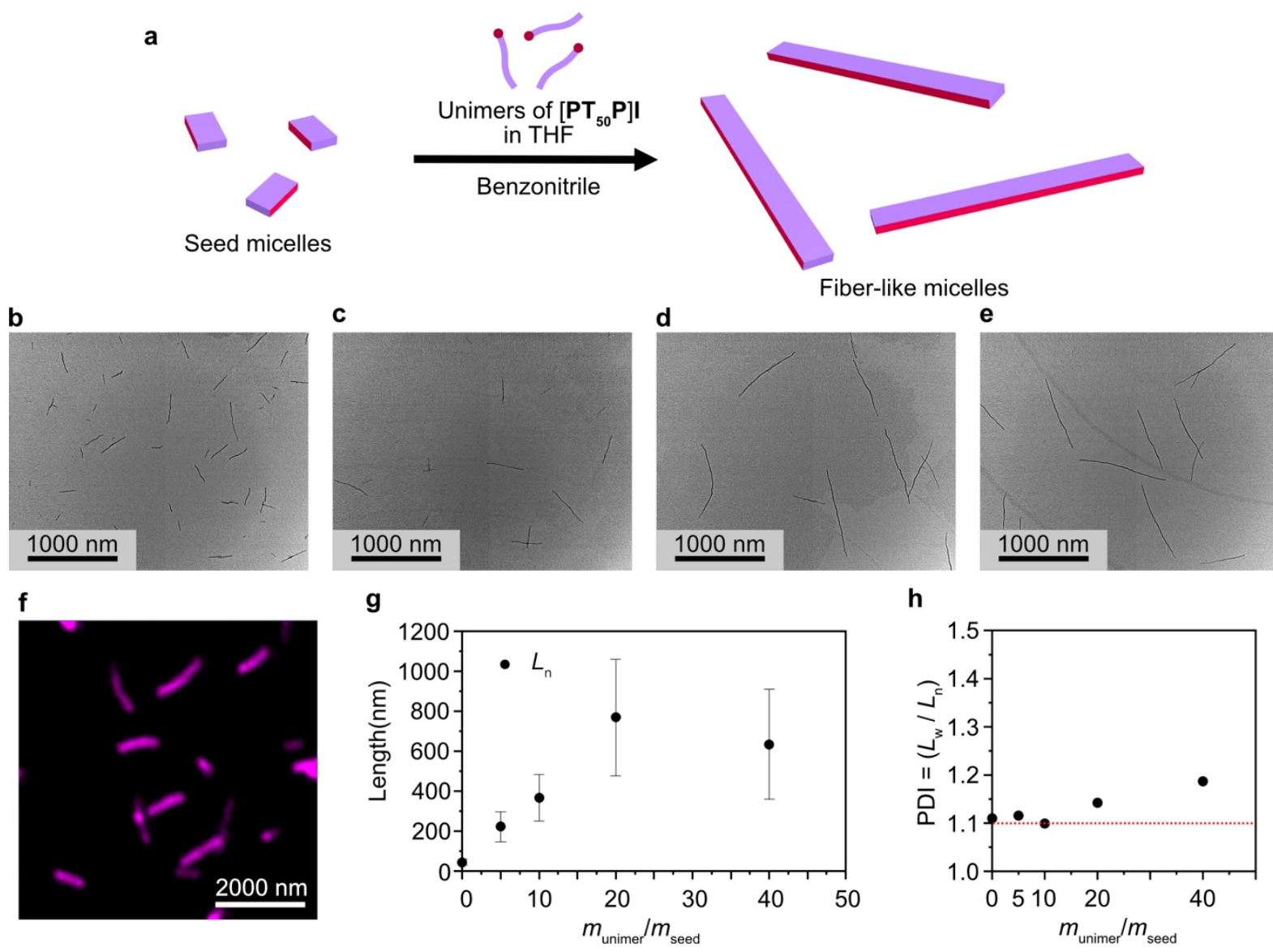

Figure S20.

(a) Schematic representation of living CDSA. (b-e) TEM images of micelles of $\left[\mathbf{P T} \mathbf{5}_{\mathbf{5 0}} \mathbf{P}\right] \mathbf{I}$ formed by living CDSA; unimer to seed ratio $m_{\text {unime }} / m_{\text {sed }}=5$ (b), 10 (c), 20 (d), and 40 (e). (f) CLSM image of length-controlled micelles of $\left[\mathbf{P} \mathbf{T}_{50} \mathbf{P}\right] \mathbf{I}$ in benzonitrile $($ Conc. $=0.02 \mathrm{mg} / \mathrm{mL})$. (g) Number-average length $\left(L_{\mathrm{n}}\right)$ of micelles of $\left[\mathbf{P} \mathbf{T}_{\mathrm{s} \mathbf{P}} \mathbf{P}\right] \mathbf{I}$ vs unimer to seed ratio $\left(m_{\text {unima }} / m_{\text {sed }}\right)$. Error bars derived from $\pm \sigma$ (standard deviation) of the micelle length distribution. (h) Polydispersity index $(\nexists)$ of micelles of $\left[\mathbf{P} \mathbf{T}_{\mathrm{s} 0} \mathbf{P}\right] \mathbf{I}$ vs unimer to seed ratio $\left(m_{\text {unines }} / m_{\text {sec }}\right)$. 


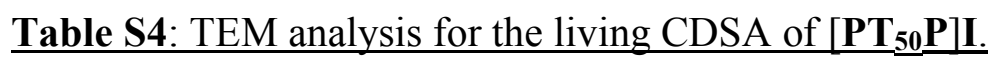

\begin{tabular}{|c|c|c|c|c|}
\hline $\boldsymbol{m}_{\text {unimer }} / \boldsymbol{m}_{\text {seed }}$ & $\boldsymbol{L}_{\mathbf{n}}(\mathbf{n m})$ & $\boldsymbol{L}_{\mathbf{w}}(\mathbf{n m})$ & $\boldsymbol{O}$ & $\boldsymbol{\sigma}(\mathbf{n m})$ \\
\hline $\mathbf{0}$ & 45.2 & 50.1 & 1.11 & 15 \\
\hline $\mathbf{5}$ & 222 & 248 & 1.12 & 76 \\
\hline $\mathbf{1 0}$ & 367 & 404 & 1.10 & 116 \\
\hline $\mathbf{2 0}$ & 768 & 878 & 1.14 & 290 \\
\hline $\mathbf{4 0}$ & 634 & 752 & 1.19 & 274 \\
\hline
\end{tabular}


Length distributions of the resulting fiber-like micelles derived from $\left[\mathbf{P T}_{\underline{\mathbf{5 0}}} \underline{\mathbf{P}}\right] \mathbf{I}$

a

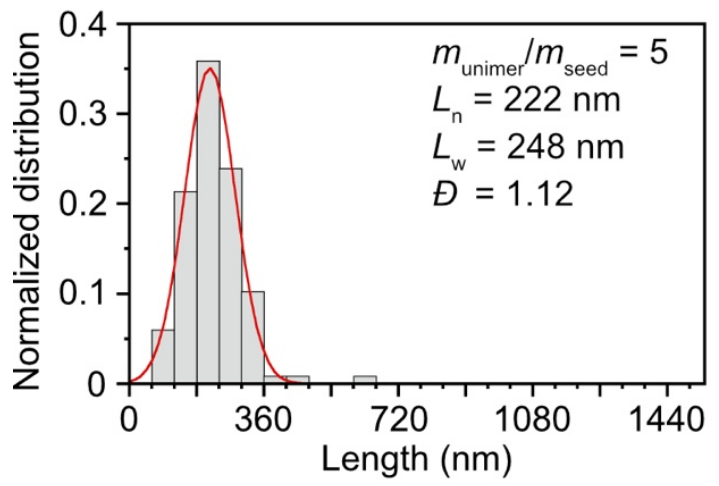

C

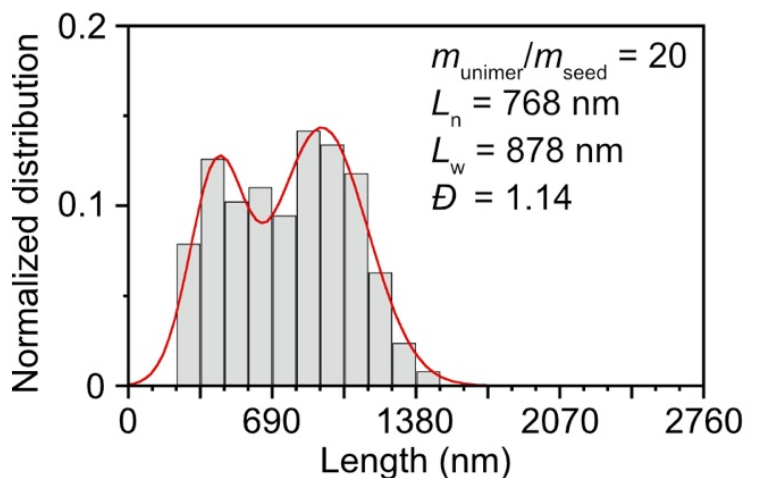

b

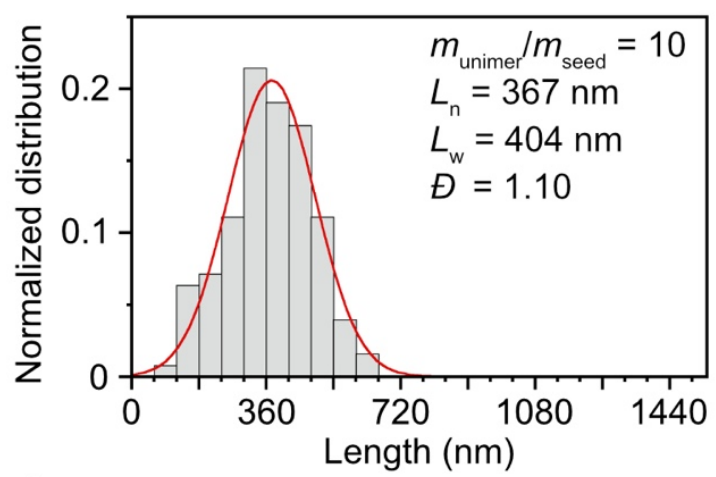

d

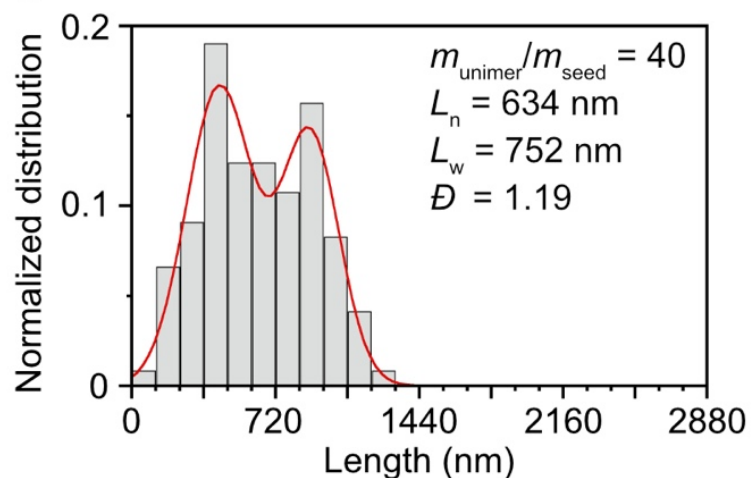

Figure S21.

Histogram of the length of resulting micelles formed by living CDSA (number-average length of seed micelles was $45 \mathrm{~nm}$ as shown in Figure S20): unimer to seed ratio $\left(m_{\text {unimer }} / m_{\text {seed }}\right)=($ a) 5, (b) 10, (c) 20, and (d) 40. Red lines were the results of the fitting based on Gaussian function (a and b, Eq. 4) or bivariate Gaussian function (c and d, Eq. 5).

$$
f(x)=a \cdot \exp \left\{-\frac{\left(x-L_{A V G}\right)^{2}}{2 \sigma^{2}}\right\}
$$

Eq. 4

$$
f(x)=a\left[\alpha \exp \left\{-\frac{\left(x-L_{\alpha A V G}\right)^{2}}{2 \sigma_{\alpha}{ }^{2}}\right\}+\beta \exp \left\{-\frac{\left(x-L_{\beta A V G}\right)^{2}}{2 \sigma_{\beta}^{2}}\right\}\right]
$$

Eq. 5 
$\underline{\text { Table S5: The statistical analysis on the histogram of }[\mathbf{P T}} \underline{\mathbf{5 0}} \underline{\mathbf{P}] \mathbf{I}}$

\begin{tabular}{|c|c|c|c|c|c|c|c|}
\hline$m_{\text {unimer }} / m_{\text {seed }}$ & $R^{2}$ & $\alpha$ & $\begin{array}{c}\boldsymbol{L}_{\alpha} \text { avg } \\
(\mathrm{nm})\end{array}$ & $\sigma_{\alpha}(\mathrm{nm})$ & $\beta$ & $\begin{array}{c}\boldsymbol{L}_{\boldsymbol{\beta} \text { avg }} \\
(\mathrm{nm})\end{array}$ & $\sigma_{\beta}(\mathrm{nm})$ \\
\hline $\mathbf{0}$ & 0.962 & 1 & 44.1 & 19 & - & - & - \\
\hline 5 & 0.995 & 1 & 216 & 68 & - & - & - \\
\hline 10 & 0.965 & 1 & 375 & 116 & - & - & - \\
\hline 20 & 0.978 & 0.552 & 929 & 220 & 0.448 & 424 & 130 \\
\hline 40 & 0.983 & 0.457 & 893 & 145 & 0.543 & 439 & 165 \\
\hline
\end{tabular}




\section{Kinetic analysis of the living CDSA}

To understand the kinetic order of the seeded growth living CDSA process we attempted to evaluate the kinetics by using data obtained by using UV/vis absorption spectroscopy. Unlike the small molecular self-assembly, unimers possess a molar mass distribution. However, in this study, we have used polymers having a low dispersity $(\nexists=1.04$ or 1.07$)$. Therefore, we assumed that the number-average molar mass $M_{\mathrm{n}}$ is the molar mass of the polymers. In general, the seeded self-assembly process can be described by equation 6 , where $K_{\mathrm{E}}$ is an equilibrium constant on the growth process and $k_{+}$and $k$ are rate constants for the formation and dissociation of micelle, respectively ${ }^{\mathrm{s} 3-6}$.

$$
\begin{gathered}
\text { unimer }+ \text { micelle }_{n}(\text { seed }) \stackrel{k_{+}}{\rightleftharpoons} \text { micelle }_{n+1} \\
k_{-} \\
K_{E}=\frac{k_{+}}{k_{-}}
\end{gathered}
$$

We have previously reported that dissociation of micelles with crystalline cores was not observed over time. Therefore, the rate constant $k$ can be regarded as negligible, and thus, seeded self-assembly is described as Eq. 7.

$$
\text { unimer }+ \text { seed } \stackrel{k}{\rightarrow} \text { micelle }
$$

The initial self-assembly rate $v_{0}$ on the seeded self-assembly can be described as Eq. 8, where the reaction order is $a$. The initial self-assembly rate $v_{0}$ has a relationship with the molar absorption coefficienct $\varepsilon$, which is calculated based on the number-average molar mass $M_{\mathrm{n}}$.

$$
\begin{gathered}
v_{0}=k[\text { unimer }][\text { seed }]^{a} \\
v_{0} \propto-\frac{d \epsilon}{d t}
\end{gathered}
$$

By taking the logarithm of both sides, the relational expression between the initial selfassembly rate $v_{0}$ and the reaction order $a$ can be derived as Eq. 9 .

$$
\log v_{0}=\log \left\{k[\text { unimer }][\text { seed }]^{a}\right\}
$$

If the concentration of unimer is much higher than that of seed, Eq. 10 can be described as follows:

$$
\log \left(-\frac{d \epsilon}{d t}\right) \propto a \log [\text { seed }]+b
$$


For further analysis, we also evaluate the kinetics on the seeded growth process based on the other equation as follows. The time-course of the change of unimer concentration can be derived as Eq. 11 from Eq. 6.

$$
-\frac{d[\text { unimer }]}{d t}=k_{+}[\text {unimer }][\text { seed }]-k_{-}[\text {seed }]
$$

Eq. 11 can be expressed as follow.

$$
-\frac{d[\text { unimer }]}{d t}=k_{+}[\text {seed }]\left([\text { unimer }]-[\text { unimer }]_{\infty}\right)
$$

In Eq. 12, [unimer] $]_{\infty}$ is defined as $k-/ k+$ which corresponds to the concentration of the unimer coexisting with micelles at the equilibrium. Integral of Eq. 12 gives Eq. 13 as follows, where [unimer $]_{0}$ corresponds to the initial concentration of the unimer.

$$
[\text { unimer }]-[\text { unimer }]_{\infty}=\left([\text { unimer }]_{0}-[\text { unimer }]_{\infty}\right) \exp \left(-k_{+}[\text {seed }] t\right)
$$

As noted above, dissociation of the micelles with crystalline core was not observed over time, and thus, the concentration of the unimer coexisting at the equilibrium is sufficiently small compared to the initial concentration of the unimer. Therefore, Eq. 12 can be approximated to Eq. 14 at the initial process.

$$
[\text { unimer }]=[\text { unimer }]_{0} \exp \left(-k_{+}[\text {seed }] t\right)
$$

By taking the logarithm of both sides, Eq. 15 can be derived from Eq. 14.

$$
\log [\text { unimer }]=-k_{+}[\text {seed }] t+b
$$

For the kinetic analyses on the seeded growth process, we used Eqs. 10 and 15. 
Kinetic studies of the living CDSA process for $\left[\mathbf{P T}_{\mathbf{3}} \mathbf{P}\right] \mathbf{I}$ in benzonitrile.

a

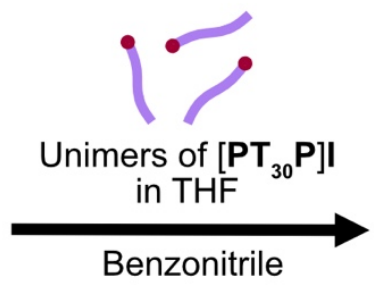

Seed micelles

b

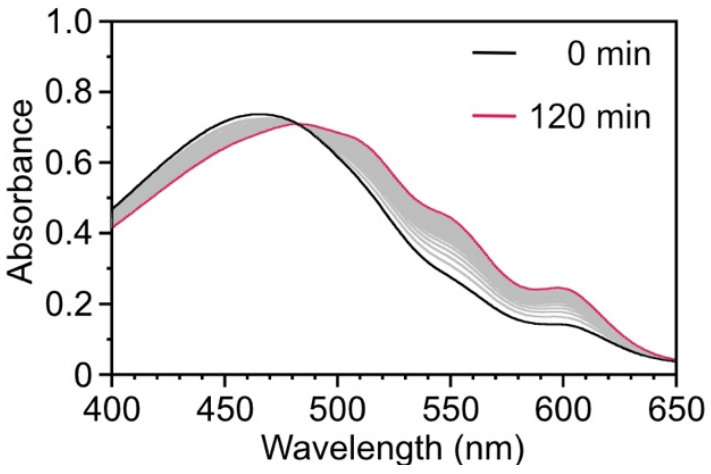

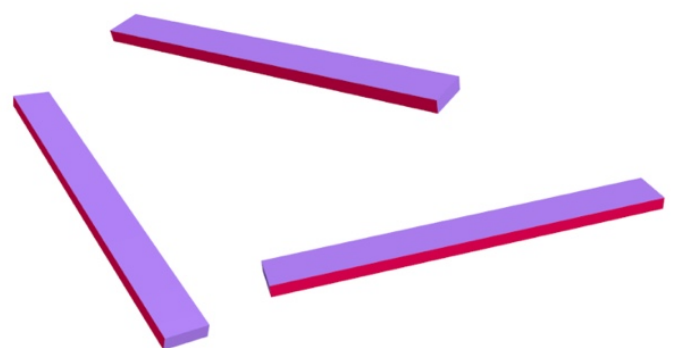

Fiber-like micelles

C

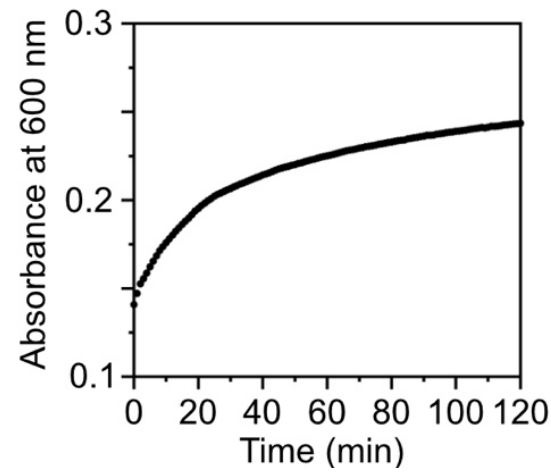

Figure S22.

(a) Schematic representation of seeded growth process for $\left[\mathbf{P T}_{30} \mathbf{P}\right] \mathbf{I}$ at $35^{\circ} \mathrm{C}$ which was monitored by UV/vis absorption spectroscopy. (b) Time-dependent absorption spectral change after the addition of the unimer solution to the solution of seed micelles: unimer to seed ratio $\left(m_{\text {unimer }} / m_{\text {seed }}\right)=10$, Total concentration of $\left[\mathbf{P T} \mathbf{T}_{\mathbf{3 0}} \mathbf{P}\right] \mathbf{I}=0.1 \mathrm{mg} / \mathrm{mL}$. (c) Time-dependent change in absorbance at $600 \mathrm{~nm}$ of $\left[\mathbf{P} \mathbf{T}_{30} \mathbf{P}\right] \mathbf{I}$ after the addition of unimers to the seeds. 
a

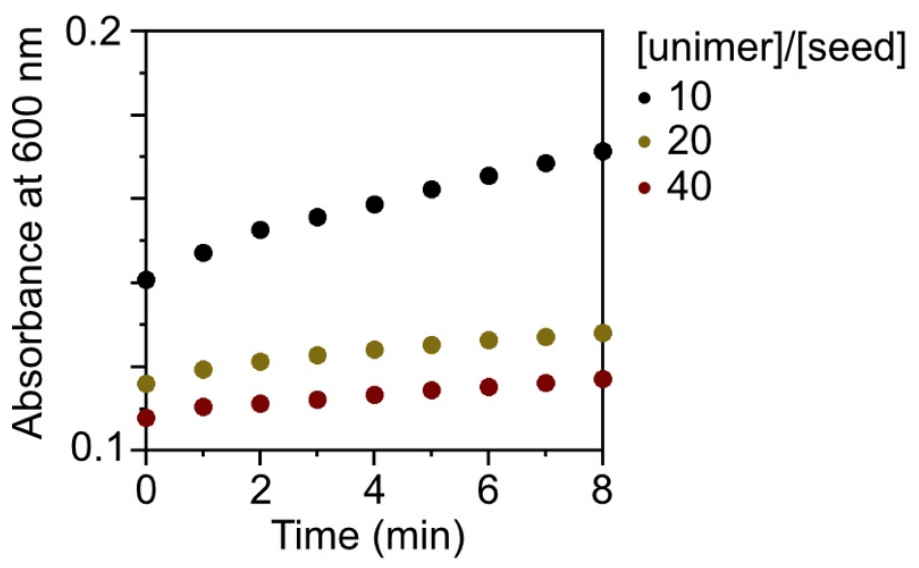

C

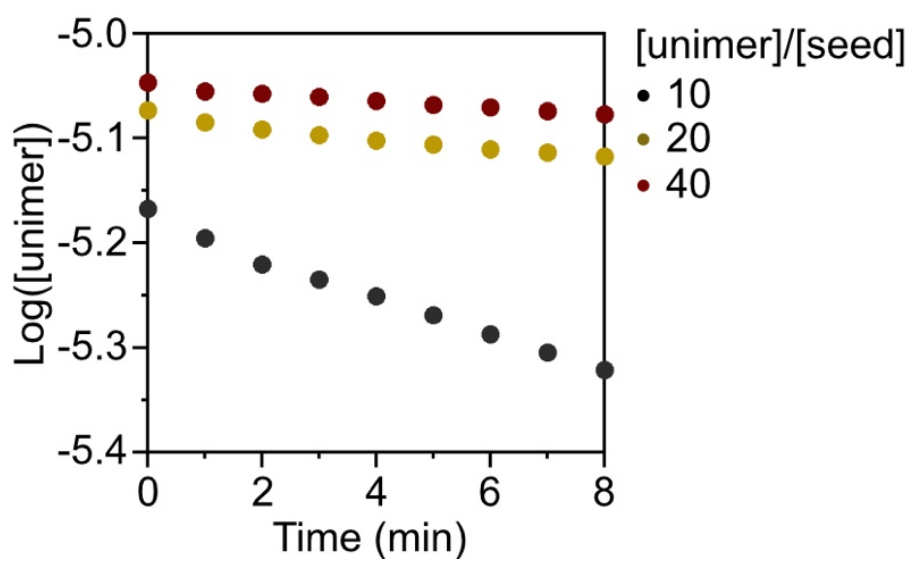

b

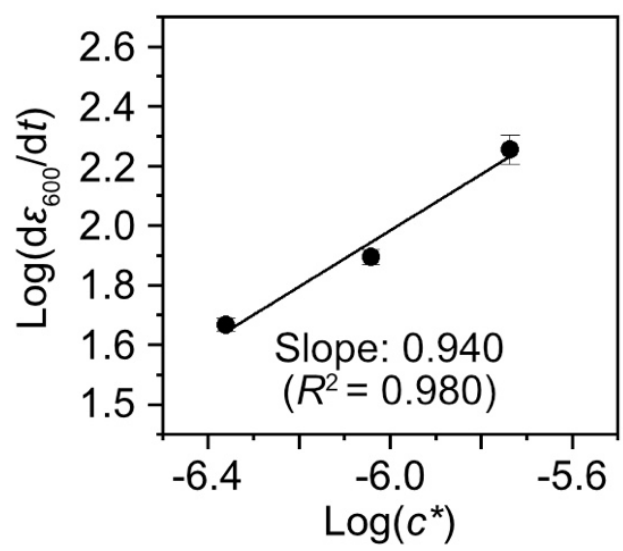

d

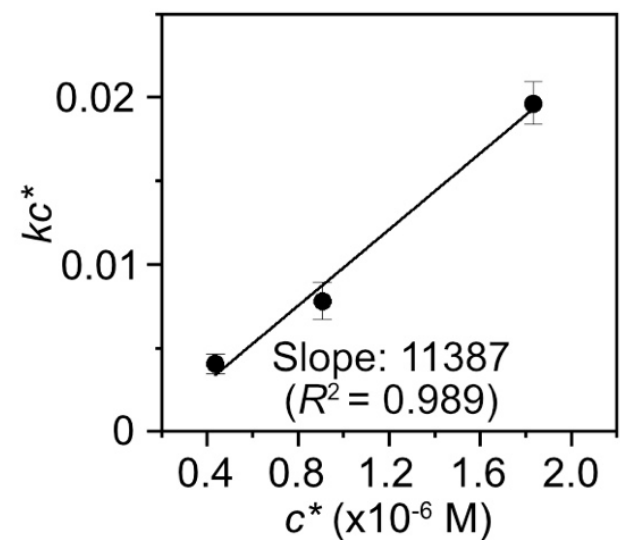

Figure S23.

(a) Plot of the change in absorbance at $600 \mathrm{~nm}$ upon the addition of the THF solution of [PT $\left.\mathbf{T}_{30} \mathbf{P}\right] \mathbf{I}$ unimers to the benzonitrile solution of seed micelles. (b) Log- log plot of the rate of the increase of molar absorption coefficient at $600 \mathrm{~nm}$ as a function of the concentration of the seed micelles. This process was evaluated by Eq. 10. Error bars derived from $\pm \sigma$ (standard deviation) of the logarithm of apparent growth rate. (c) Plot of the change in the concentration of the unimers upon the addition of the unimers to the seed micelles. (d) Plot of the $k c^{\circ}$ as a function of the concentration of the seed micelles. This was evaluated by Eq. 15. Error bars derived from $\pm \sigma$ (standard deviation) of $k c^{*}$.

$$
\begin{gathered}
\log \left(-\frac{d \epsilon}{d t}\right) \propto a \cdot \log c^{*}+b \\
\log [\text { unimer }]=-k c^{*} t+b
\end{gathered}
$$

In the above equations, $c^{*}$ is the concentration of the seed micelles. 
Kinetic studies of the living CDSA process for $[\mathbf{P T} \underline{\mathbf{5 0}} \mathbf{P}] \mathbf{I}$ in benzonitrile.

a

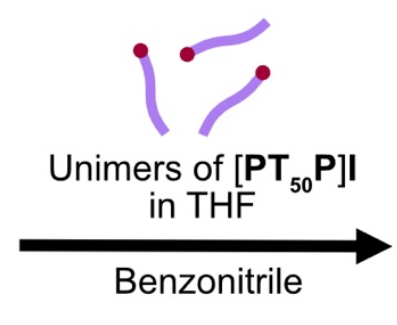

Seed micelles

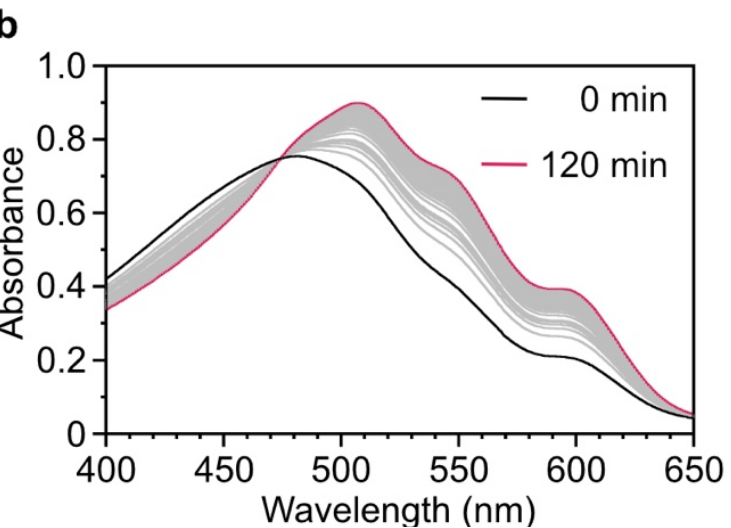

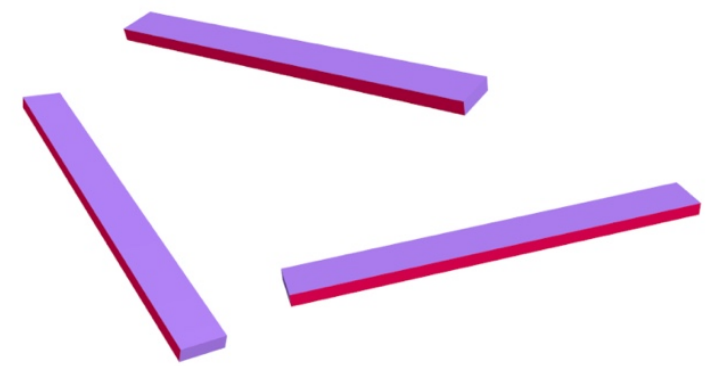

Fiber-like micelles

C

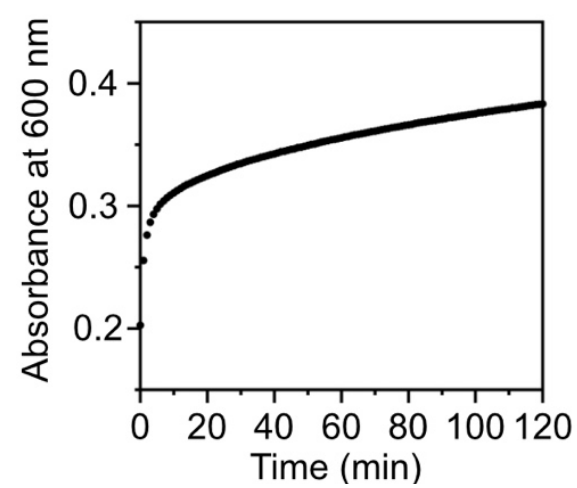

Figure S24.

(a) Schematic representation of seeded growth process for $\left[\mathbf{P T}_{50} \mathbf{P}\right] \mathbf{I}$ at $35{ }^{\circ} \mathrm{C}$ which was monitored by UV/vis absorption spectroscopy. (b) Time-dependent absorption spectral change after the addition of the unimer solution to the solution of seed micelles: unimer to seed ratio $\left(m_{\text {unimer }} / m_{\text {seed }}\right)=10$, Total concentration of $\left[\mathbf{P T}_{\mathbf{5 0}} \mathbf{P}\right] \mathbf{I}=0.1 \mathrm{mg} / \mathrm{mL}$. (c) Time-dependent change in absorbance at $600 \mathrm{~nm}$ of $\left[\mathbf{P} \mathbf{T}_{\mathbf{s}_{0}} \mathbf{P}\right] \mathbf{I}$ after the addition of unimers to the seeds. 
a

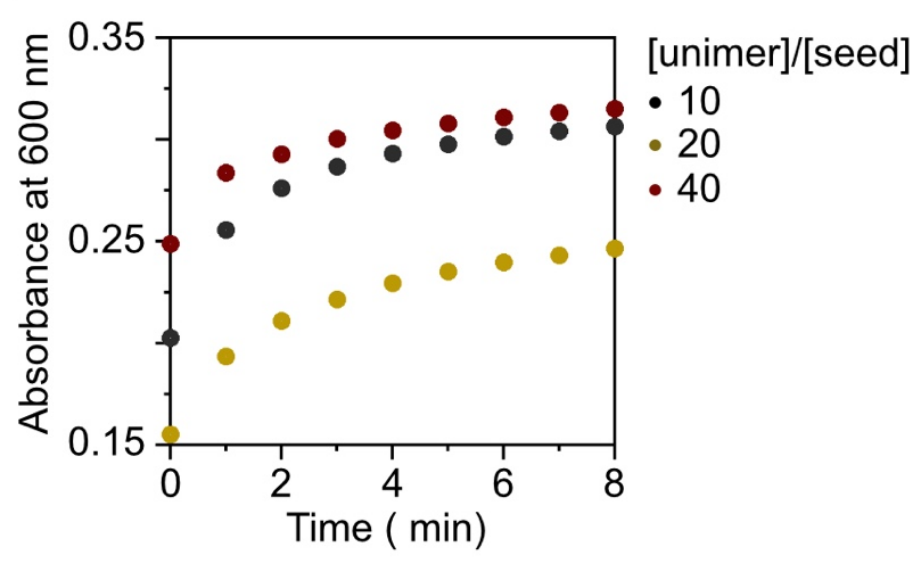

C

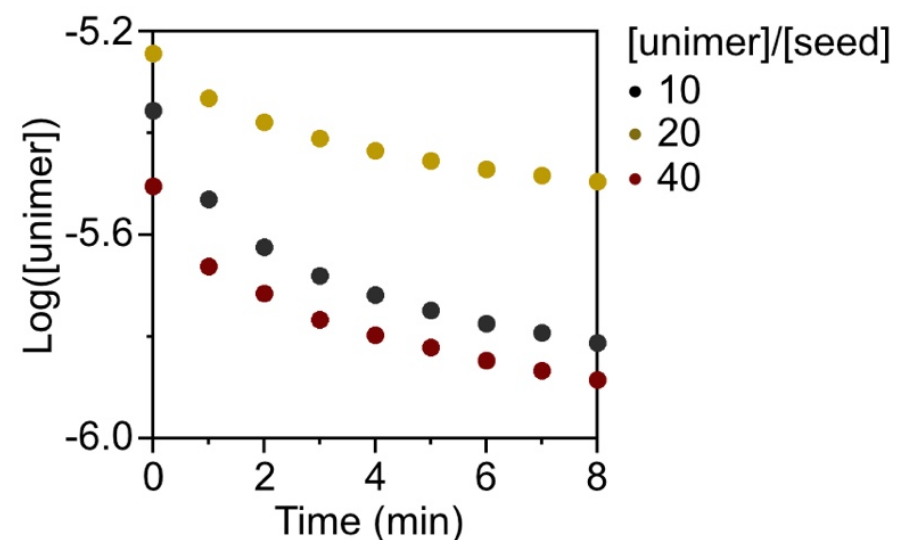

b

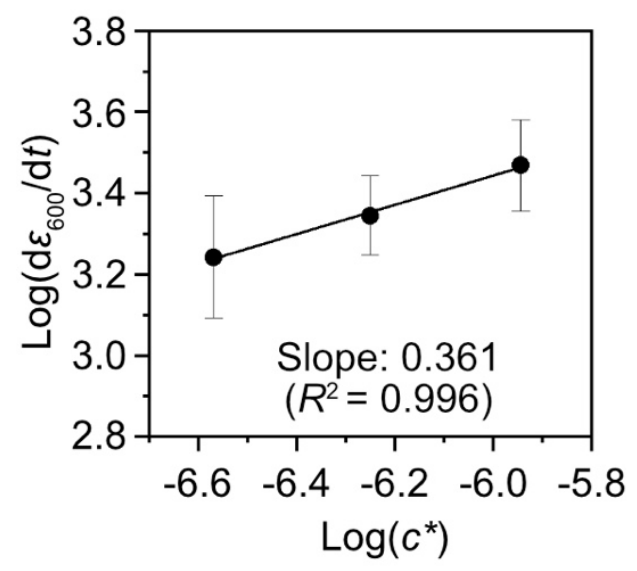

d

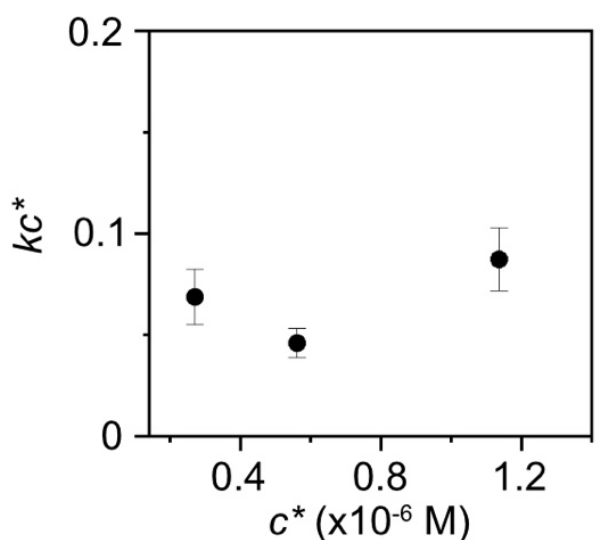

Figure S25.

(a) Plot of the change in absorbance at $600 \mathrm{~nm}$ upon the addition of THF solution of $\left[\mathbf{P T} \mathbf{T}_{50} \mathbf{P}\right] \mathbf{I}$ unimer to the benzonitrile solution of seed micelles. (b) Log-log plot of the rate of the increase of molar absorption coefficient at $600 \mathrm{~nm}$ as a function of the concentration of the seed micelles. This process was evaluated by Eq. 10. Error bars derived from $\pm \sigma$ (standard deviation) of the logarithm of apparent growth rate. (c) Plot of the change in the concentration of the unimers upon the addition of the unimers into seed micelles. (d) Plot of the $k c^{*}$ as a function of the concentration of the seed micelles. This was evaluated by Eq. 15. Error bars derived from $\pm \sigma$ (standard deviation) of $k c^{*}$.

$$
\begin{gathered}
\log \left(-\frac{d \epsilon}{d t}\right) \propto a \cdot \log c^{*}+b \\
\log [\text { unimer }]=-k c^{*} t+b
\end{gathered}
$$

In the above equations, $c^{*}$ is the concentration of the seed micelles. 
$\underline{\text { Self-assembly of }[\mathbf{P T}} \underline{\mathbf{5 0}} \mathbf{P}] \mathbf{I}$ in benzonitrile and THF mixture

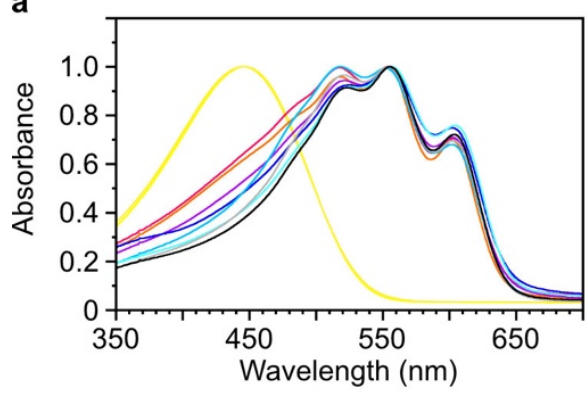

C

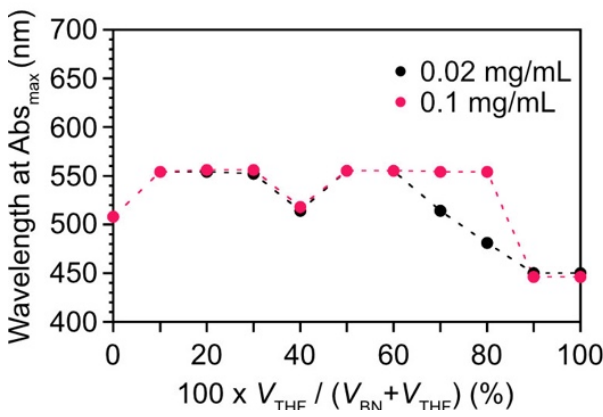

b

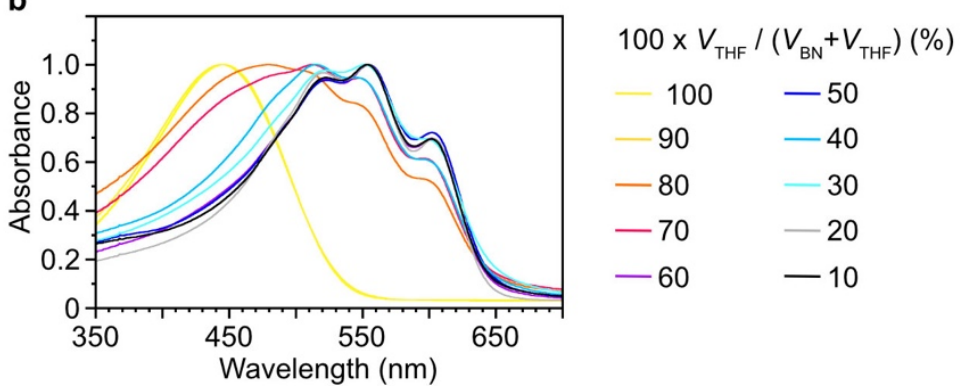

d

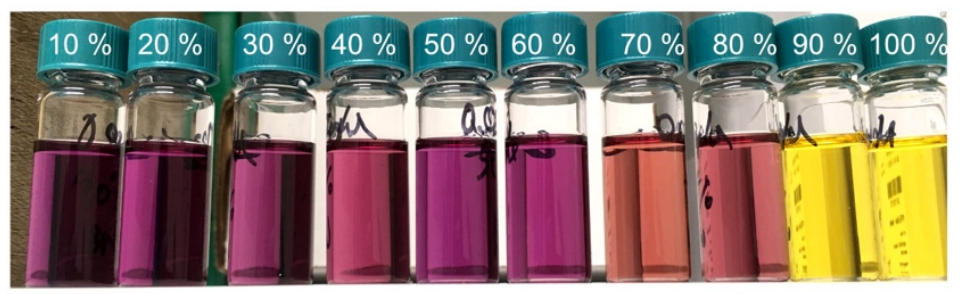

Figure S26.

(a and b) UV/vis absorption spectra of $\left[\mathbf{P T}_{\mathbf{5 0}} \mathbf{P}\right] \mathbf{I}$ in benzonitrile/THF mixtures after spontaneous self-assembly: Concentrations of $\left[\mathbf{P T}_{\mathbf{5 0}} \mathbf{P}\right] \mathbf{I}=0.1 \mathrm{mg} / \mathrm{mL}$ (a) and $0.02 \mathrm{mg} / \mathrm{mL}$ (b). (c) Wavelength at absorption maxima vs fractional volume \% of THF for the mixed solvent. (d) Photograph of $\left[\mathbf{P T}_{\mathbf{5 0}} \mathbf{P}\right] \mathbf{I}$ in benzonitrile/THF mixtures after the spontaneous self-assembly. 


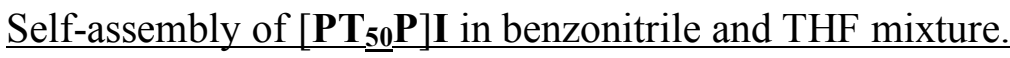<smiles>C=CC=C[PH](C)(c1ccccc1)c1ccc(C(C)(C)c2sc(C(C)(C)c3ccccc3C)cc2C)cc1</smiles>

$\left[\mathrm{PT}_{50} \mathrm{P}\right]$

b
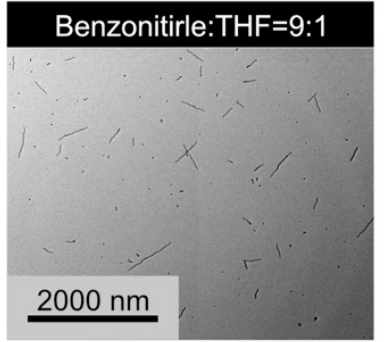

$f$

\section{Benzonitirle:THF=5:5}

$2000 \mathrm{~nm}$
C

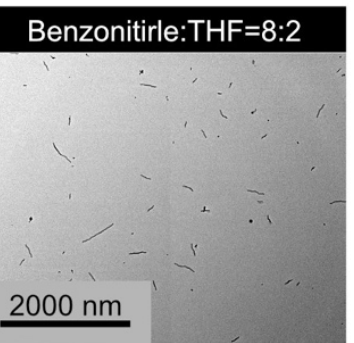

g

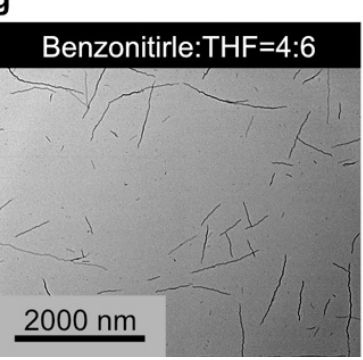

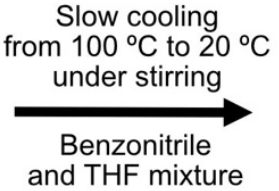
and THF mixture

Unimer

d

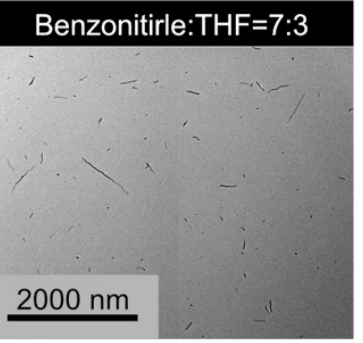

h

Benzonitirle:THF=3:7

$2000 \mathrm{~nm}$

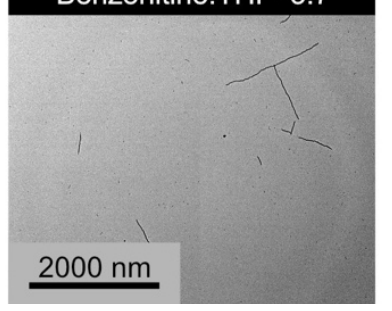

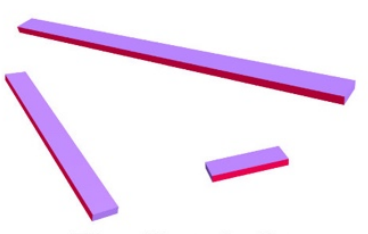

Fiber-like micelles

e

$2000 \mathrm{~nm}$

i

Figure S27.

(a) Schematic representation of self-assembly of $\left[\mathbf{P T} \mathbf{T}_{\mathbf{5 0}} \mathbf{P}\right] \mathbf{I}$ in benzonitrile and THF mixture. (b-i) TEM images of the resulting micelles of $\left[\mathbf{P T}_{\mathbf{5 0}_{0}} \mathbf{P}\right] \mathbf{I}$ in benzonitrile and THF mixtures. 
Evaluation of self-assembly of $\left[\mathbf{P T}_{\underline{\mathbf{5}} \mathbf{0}} \mathbf{P}\right] \mathbf{I}$ in benzonitrile and THF mixture (30\% THF)
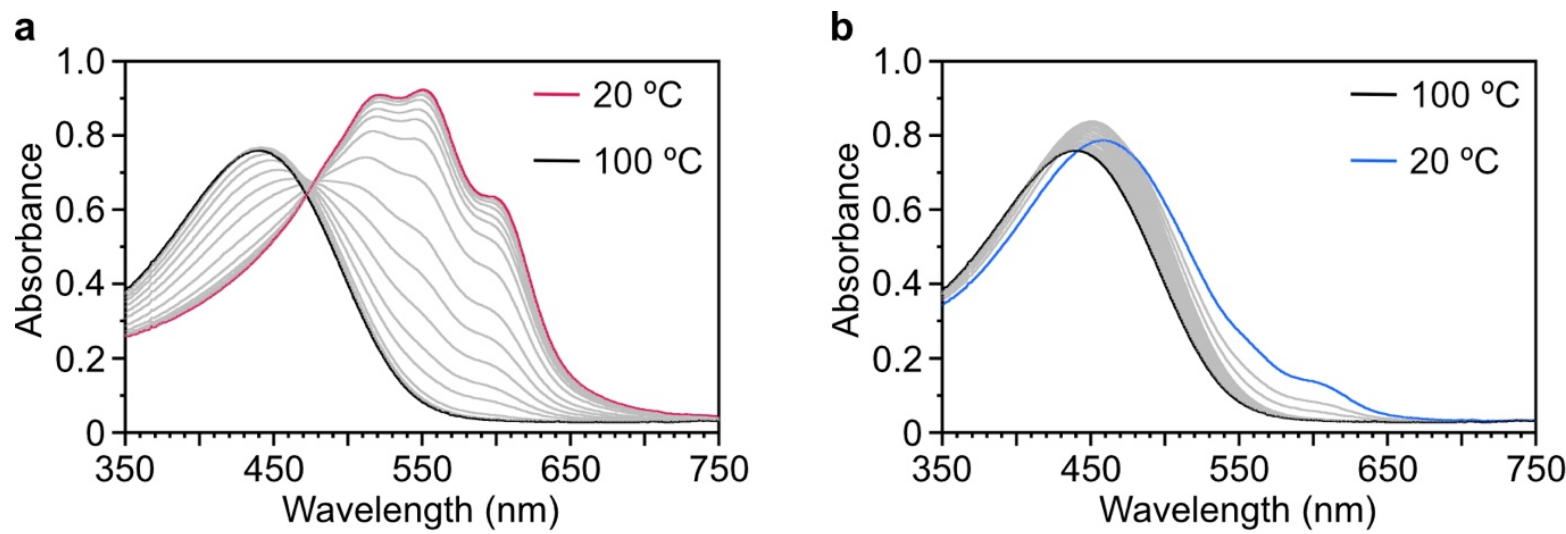

C

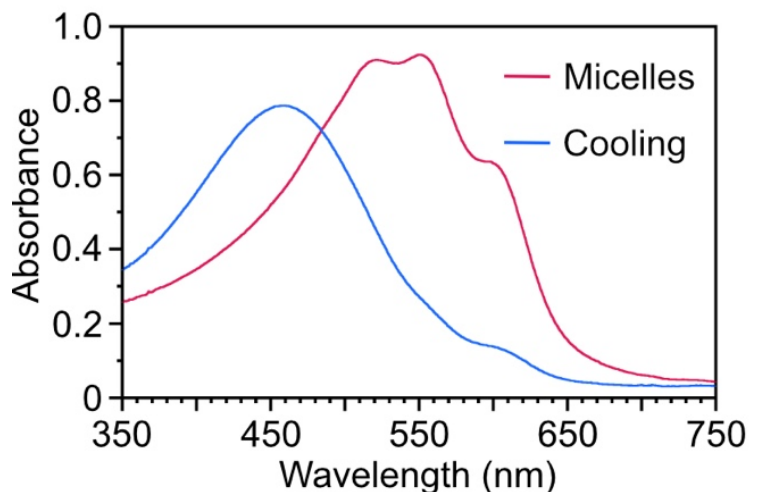

d

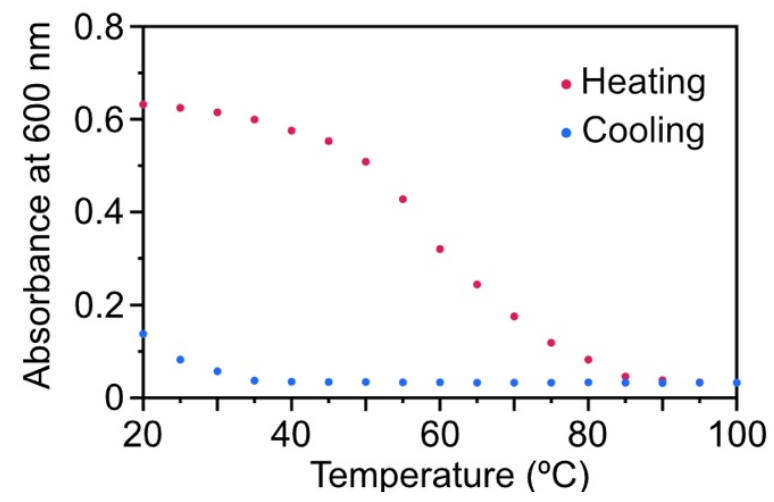

Figure S28.

(a) Temperature-dependent absorption spectra upon heating from $20^{\circ} \mathrm{C}$ to $100^{\circ} \mathrm{C}$ for a solution of $\left[\mathbf{P T}_{50} \mathbf{P}\right] \mathbf{I}$, where each spectrum was recorded at $5{ }^{\circ} \mathrm{C}$ intervals (Solvent: benzonitrile:THF $=$ $7: 3$, Heating rate: $1{ }^{\circ} \mathrm{C} / \mathrm{min}$, Conc. $=0.02 \mathrm{mg} / \mathrm{mL}$ ). (b) Temperature-dependent absorption spectra upon cooling from $100{ }^{\circ} \mathrm{C}$ to $20^{\circ} \mathrm{C}$ for a solution of $\left[\mathbf{P T} \mathbf{T}_{50} \mathbf{P}\right] \mathbf{I}$ (Solvent: benzonitrile:THF $=7: 3$, Cooling rate: $1{ }^{\circ} \mathrm{C} / \mathrm{min}$, Conc. $\left.=0.02 \mathrm{mg} / \mathrm{mL}\right)$. (c) Absorption spectra of a solution of $\left[\mathbf{P T}_{\mathrm{sp}} \mathbf{P}\right] \mathbf{I}$ at $20{ }^{\circ} \mathrm{C}$ before heating (pink) or after both heating and cooling processes (blue). (d) Temperature-dependent change in absorbance at $600 \mathrm{~nm}$ of $\left[\mathbf{P} \mathbf{T}_{50} \mathbf{P}\right] \mathbf{I}$. 
Evaluation of self-assembly of $\left[\mathbf{P T}_{\underline{\mathbf{5}} \mathbf{0}} \mathbf{P}\right] \mathbf{I}$ in benzonitrile and THF mixture (50\% THF)
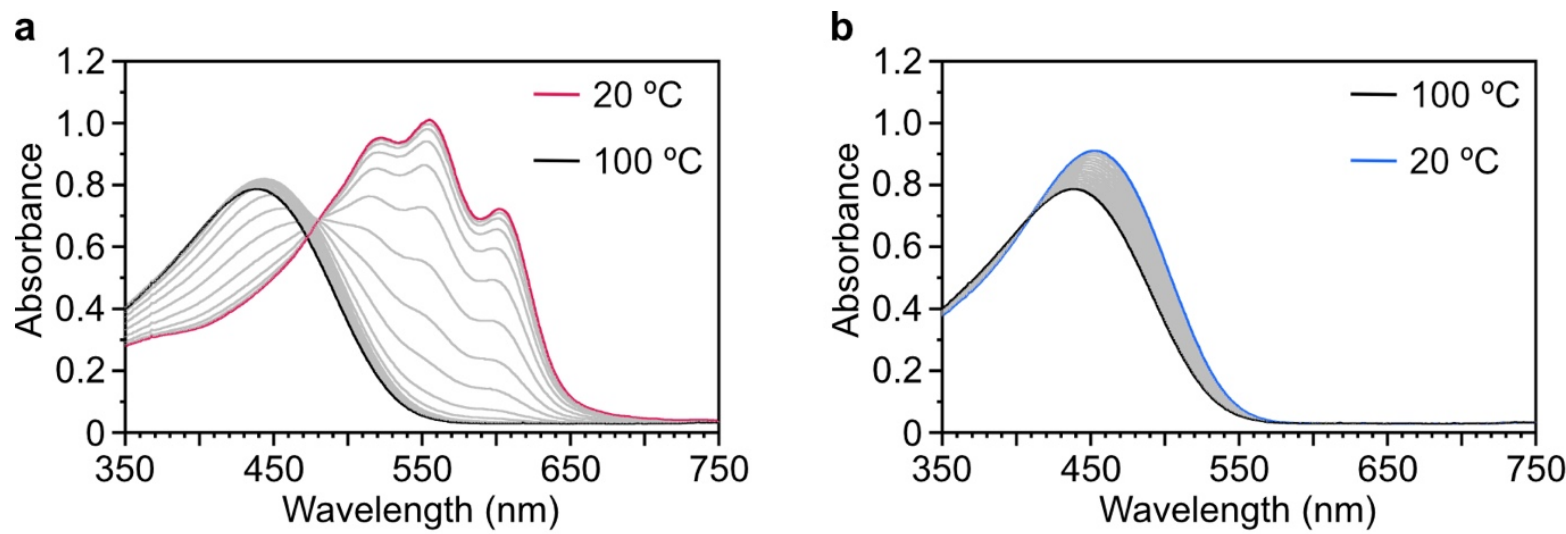

C

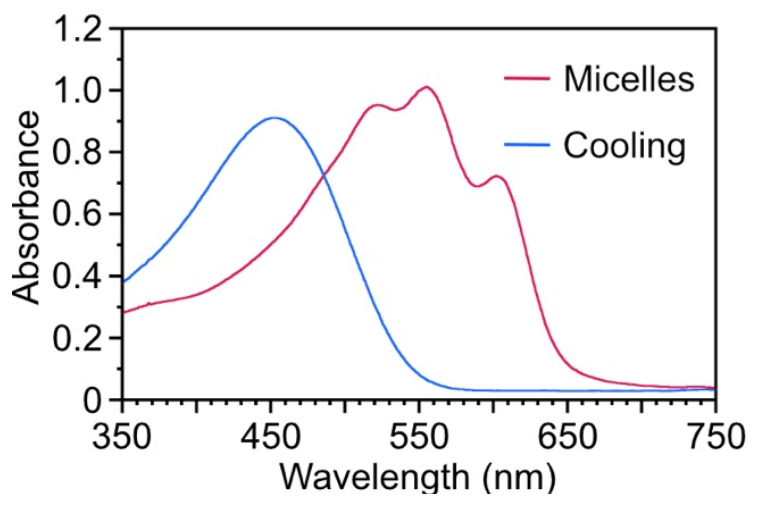

d

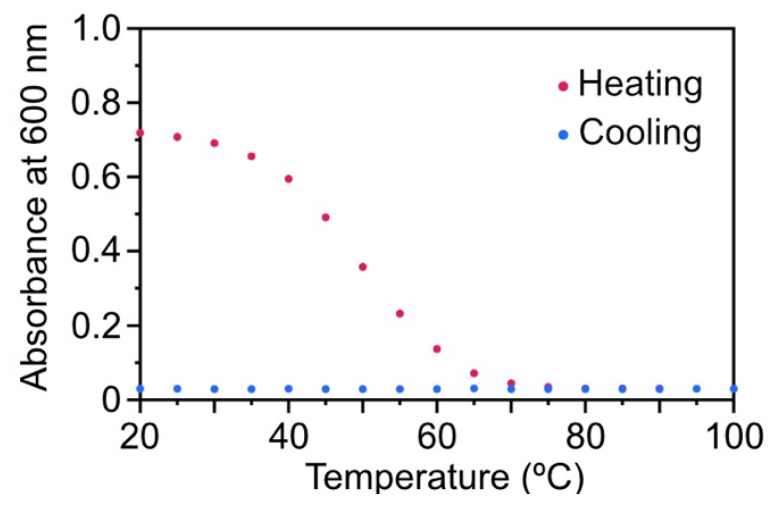

Figure S29.

(a) Temperature-dependent absorption spectra upon heating from $20^{\circ} \mathrm{C}$ to $100^{\circ} \mathrm{C}$ for a solution of $\left[\mathbf{P T}_{50} \mathbf{P}\right] \mathbf{I}$, where each spectrum was recorded every $5{ }^{\circ} \mathrm{C}$ interval (Solvent: benzonitrile:THF $=5: 5$, Heating rate: $1{ }^{\circ} \mathrm{C} / \mathrm{min}$, Conc. $=0.02 \mathrm{mg} / \mathrm{mL}$ ). (b) Temperature-dependent absorption spectra upon cooling from $100{ }^{\circ} \mathrm{C}$ to $20^{\circ} \mathrm{C}$ for a solution of $\left[\mathbf{P T} \mathbf{T}_{50} \mathbf{P}\right] \mathbf{I}$ (Solvent: benzonitrile:THF $=5: 5$, Cooling rate: $1{ }^{\circ} \mathrm{C} / \mathrm{min}$, Conc. $\left.=0.02 \mathrm{mg} / \mathrm{mL}\right)$. (c) Absorption spectra of a solution of $\left[\mathbf{P T}_{\mathrm{si}} \mathbf{P}\right] \mathbf{I}$ at $20^{\circ} \mathrm{C}$ before heating (pink) or after both heating and cooling processes (blue). (d) Temperature-dependent change in absorbance at $600 \mathrm{~nm}$ of $\left[\mathbf{P T} \mathbf{s}_{50} \mathbf{P}\right] \mathbf{I}$. 
Evaluation of self-assembly of $\left[\mathbf{P T}_{\underline{\mathbf{5}} \mathbf{0}} \mathbf{P}\right] \mathbf{I}$ in benzonitrile and THF mixture (70 \% THF)
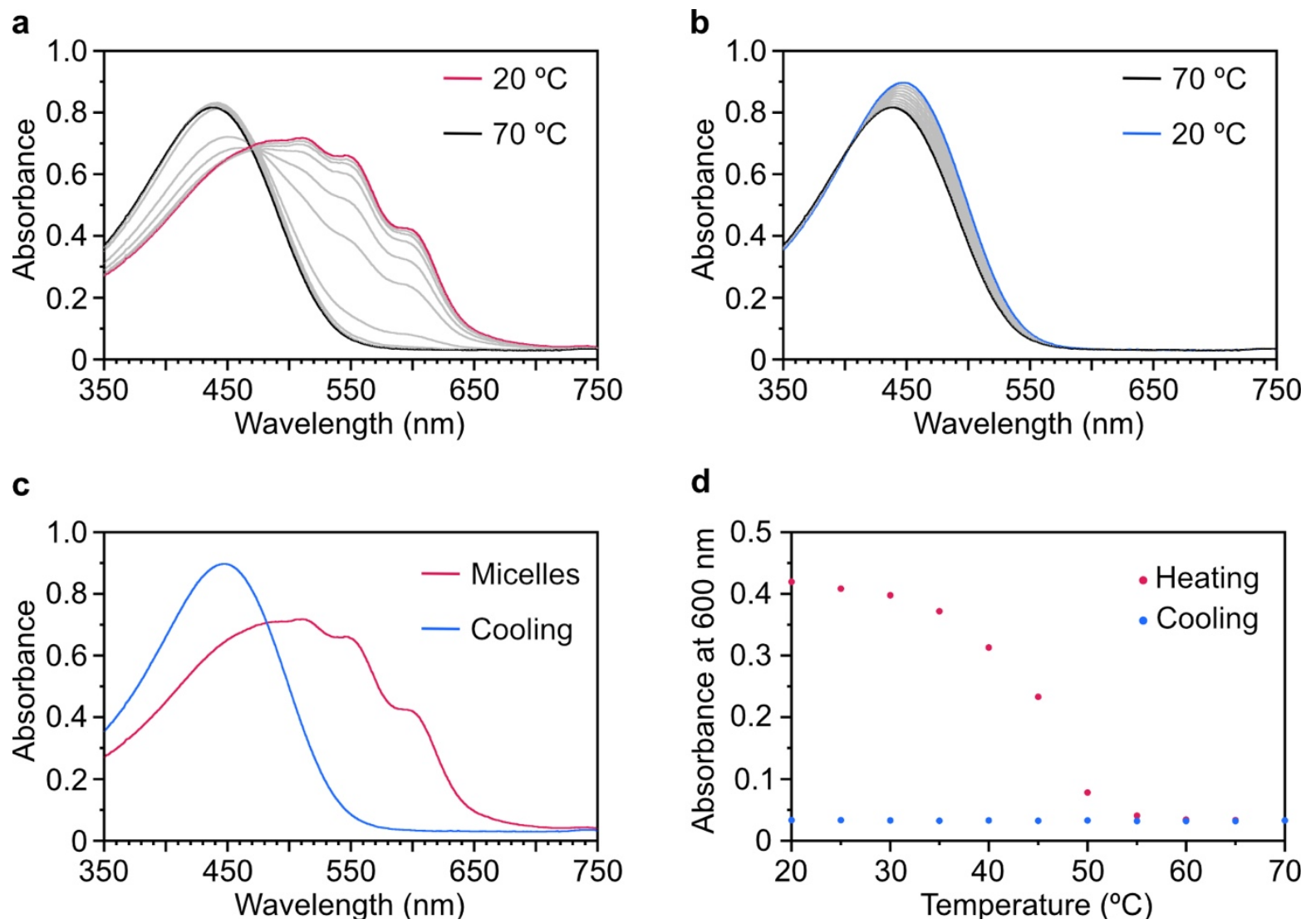

Figure S30.

(a) Temperature-dependent absorption spectra upon heating from $20{ }^{\circ} \mathrm{C}$ to $70{ }^{\circ} \mathrm{C}$ for a solution of $\left[\mathbf{P T}_{50} \mathbf{P}\right] \mathbf{I}$, where each spectrum was recorded every $5{ }^{\circ} \mathrm{C}$ interval (Solvent: benzonitrile:THF $=3: 7$, Heating rate: $1{ }^{\circ} \mathrm{C} / \mathrm{min}$, Conc. $=0.02 \mathrm{mg} / \mathrm{mL}$ ). (b) Temperature-dependent absorption spectra upon cooling from $70{ }^{\circ} \mathrm{C}$ to $20^{\circ} \mathrm{C}$ for a solution of $\left[\mathbf{P T}_{\mathrm{s} 0} \mathbf{P}\right] \mathbf{I}$ (Solvent: benzonitrile:THF $=3: 7$, Cooling rate: $1{ }^{\circ} \mathrm{C} / \mathrm{min}$, Conc. $\left.=0.02 \mathrm{mg} / \mathrm{mL}\right)$. (c) Absorption spectra of a solution of $\left[\mathbf{P T}_{\mathrm{s} 0} \mathbf{P}\right] \mathbf{I}$ at $20^{\circ} \mathrm{C}$ before heating (pink) or after both heating and cooling processes (blue). (d) Temperature-dependent change in absorbance at $600 \mathrm{~nm}$ of $\left[\mathbf{P T} \mathbf{s}_{50} \mathbf{P}\right] \mathbf{I}$. 
Injection of unimer solution into benzonitrile and THF mixture.

a

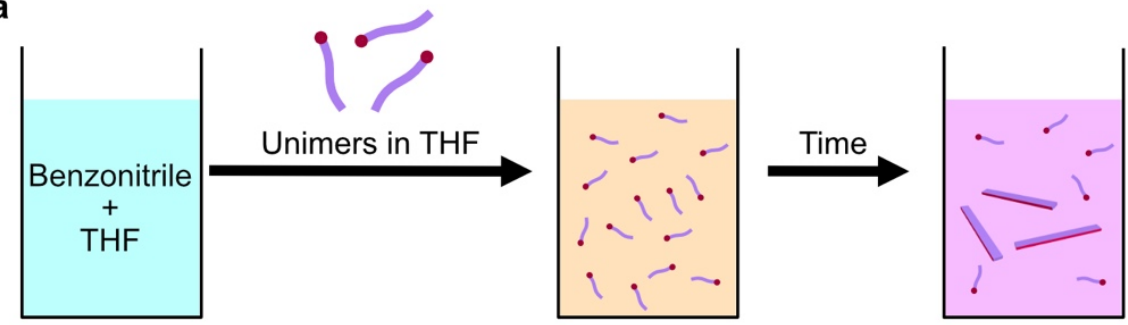

Benzonitrile:THF = 10:0

b

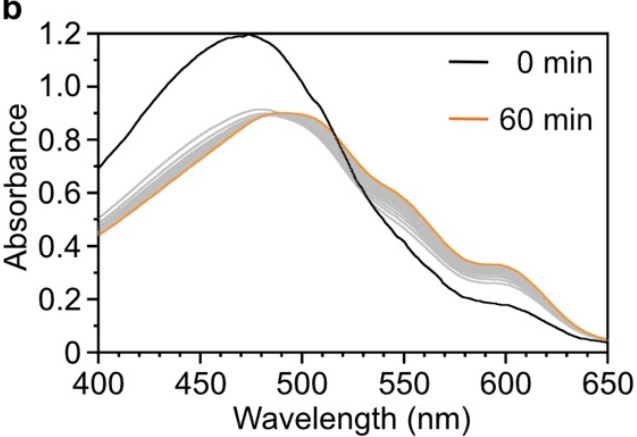

Benzonitrile:THF = 7:3

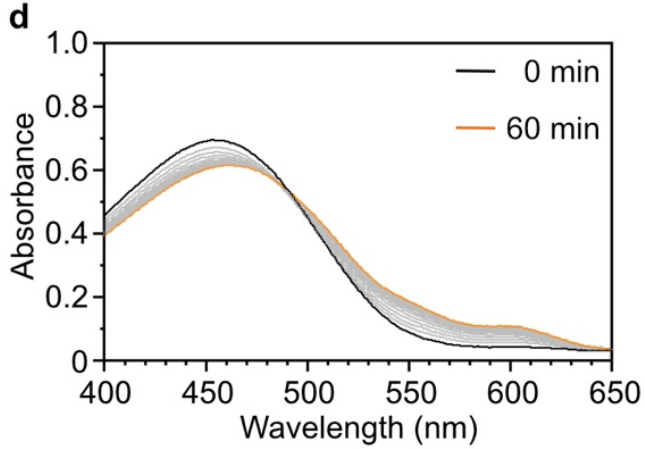

Benzonitrile:THF $=5: 5$

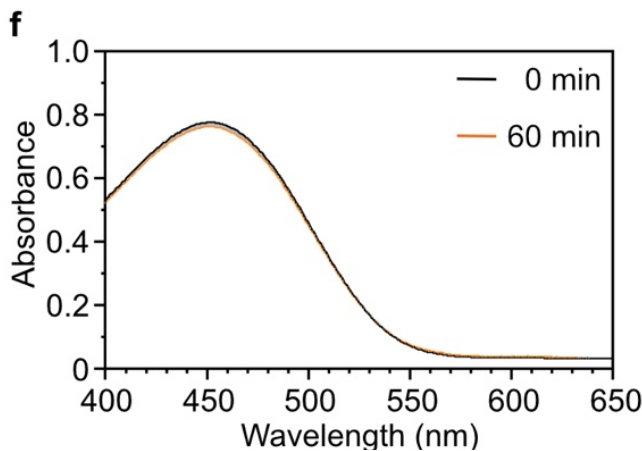

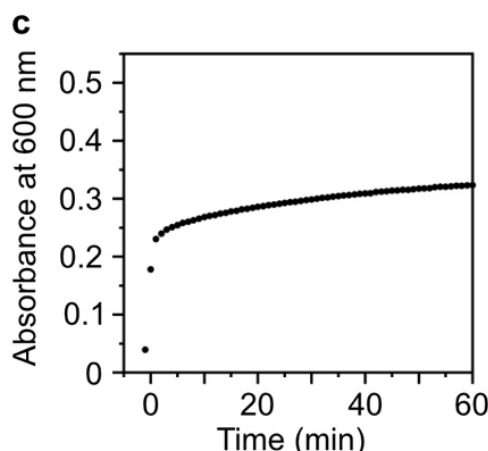
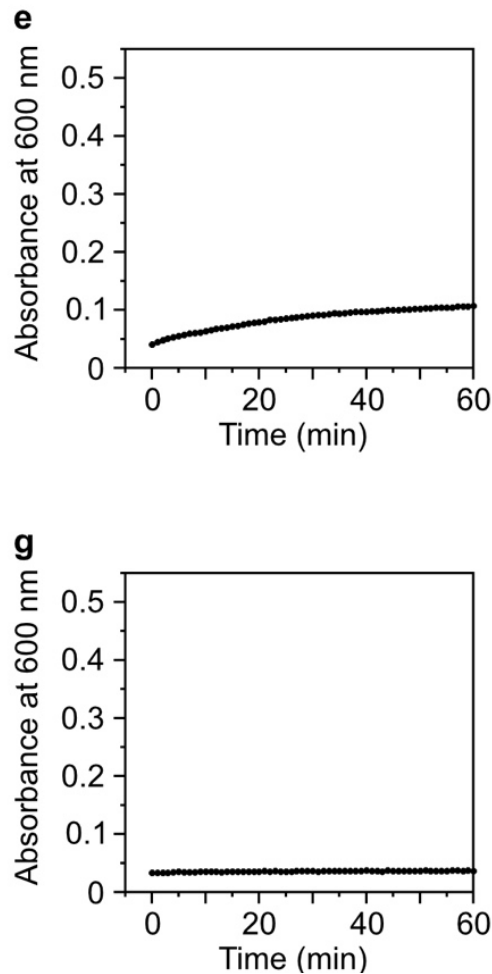

\section{Figure S31.}

(a) Schematic representation of the injection of unimers (Conc. $1 \mathrm{mg} / \mathrm{mL}$ in THF) into benzonitrile and THF mixtures at $35{ }^{\circ} \mathrm{C}$ to evaluate the self-nucleation which was monitored by UV/vis absorption spectroscopy in $1 \mathrm{~min}$ interval (final Conc. $=0.02 \mathrm{mg} / \mathrm{mL}$ ). $(\mathbf{b}, \mathbf{d}, \mathbf{f}$ ) Time-dependent absorption spectral changes after the addition of the unimers $\left[\mathbf{P T}_{\mathbf{5 0}} \mathbf{P}\right] \mathbf{I}$ to benzonitrile: Volume ratio of THF was $0(\mathbf{b}), 0.3(\mathbf{d})$, and $0.5(\mathbf{f}) .(\mathbf{c}, \mathbf{g})$ Time-dependent change in absorbance at $600 \mathrm{~nm}$ of $\left[\mathbf{P T} \mathbf{s}_{50} \mathbf{P}\right] \mathbf{I}$ after the injection of unimers: Volume ratio of THF was $0(\mathbf{c}), 0.3(\mathbf{e})$, and $0.5(\mathbf{g})$. 
Kinetic analysis on living CDSA for $[\mathbf{P T} \underline{\mathbf{5 0}} \mathbf{P}] \mathbf{I}$ in benzonitrile and THF mixture.

a
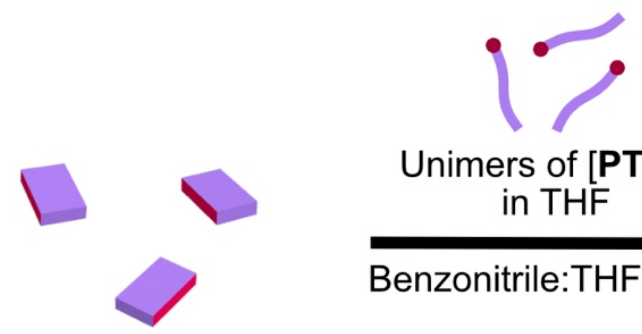

Unimers of $\left[\mathbf{P T}_{50} \mathbf{P}\right] \mathbf{I}$ in THF

Seed micelles

Benzonitrile:THF=5:5

b
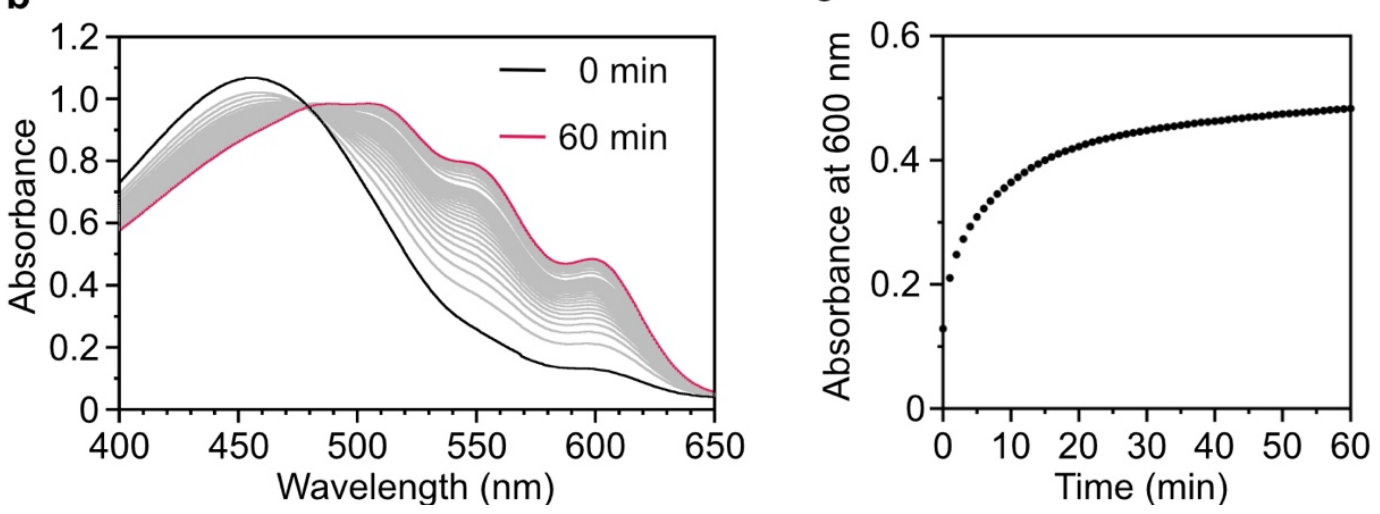

Figure S32.

(a) Schematic representation of seeded growth process at $35{ }^{\circ} \mathrm{C}$ which was monitored by UV/vis absorption spectroscopy. (b) Time-dependent absorption spectral change after the addition of the unimer solution to the solution of seed micelles: Volume percentage of THF to total volume of the solvents was $50 \%$, unimer to seed ratio $\left(m_{\text {unimer }} / m_{\text {seed }}\right)=10$, Total concentration of $\left[\mathbf{P T}_{\mathbf{5 0}} \mathbf{P}\right] \mathbf{I}=0.1 \mathrm{mg} / \mathrm{mL}$. (c) Time-dependent change in absorbance at $600 \mathrm{~nm}$ of $\left[\mathbf{P} \mathbf{T}_{\mathrm{s} 0} \mathbf{P}\right] \mathbf{I}$ after the addition of the unimers to the seeds. 
a

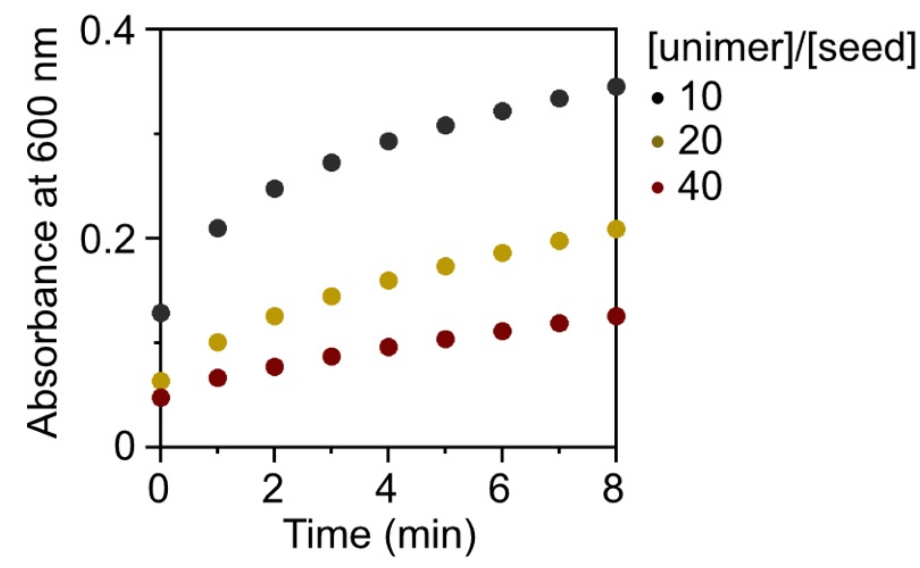

C

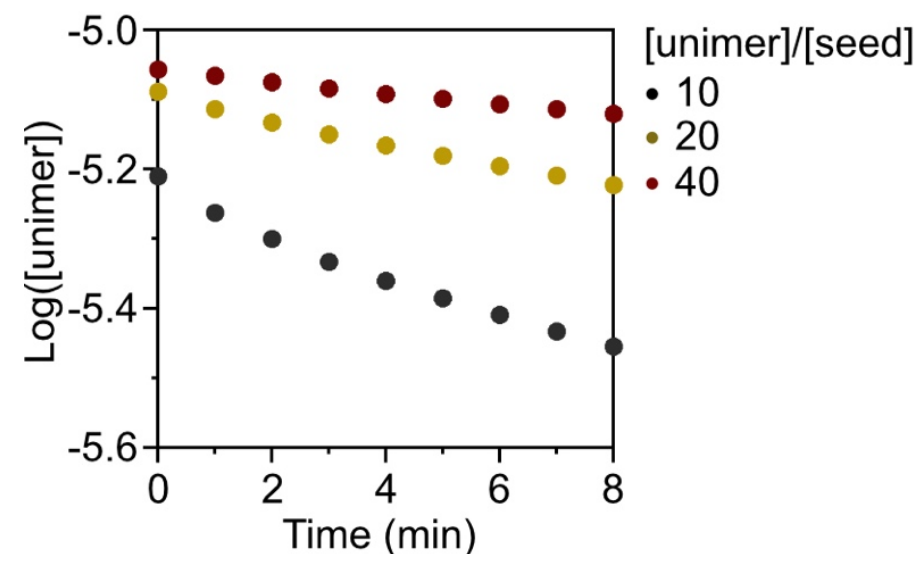

b

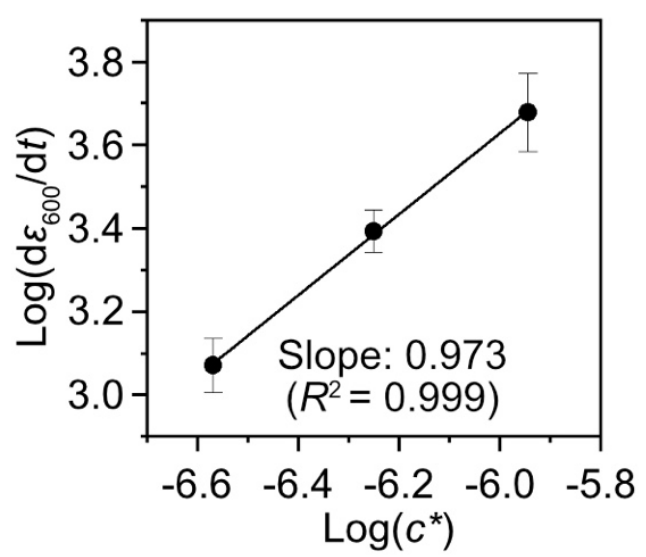

d

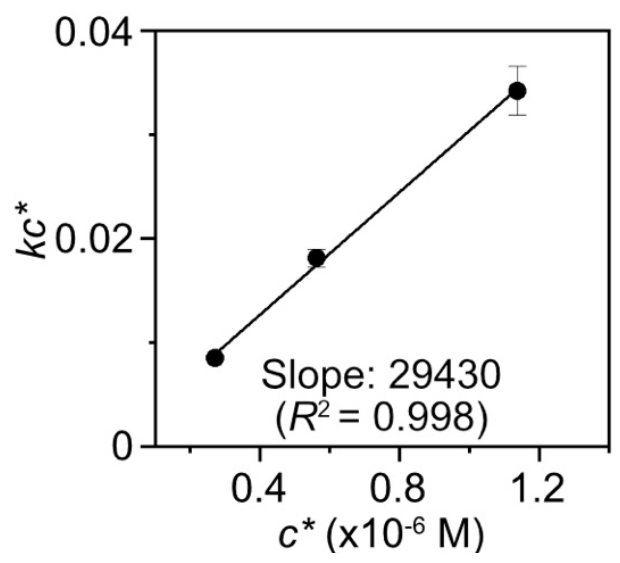

Figure S33.

(a) Plot of the change in absorbance at $600 \mathrm{~nm}$ upon the addition of THF solution of $\left[\mathbf{P T} \mathbf{5 0}_{\mathbf{5 0}} \mathbf{P}\right] \mathbf{I}$ unimers into the seed micelles in benzonitrile/THF mixture. (b) Log- log plot of the rate of the increase of molar absorption coefficient at $600 \mathrm{~nm}$ as a function of the concentration of the seed micelles. This process was evaluated by Eq. 10. Error bars derived from $\pm \sigma$ (standard deviation) for logarithm of apparent growth rate. (c) Plot of the change in the concentration of the unimers upon the addition of the unimers inot the seed micelles. (d) Plot of the $k c^{*}$ as a function of the concentration of the seed micelles. This was evaluated by Eq. 15. Error bars derived from $\pm \sigma$ (standard deviation) for $k c^{*}$.

$$
\begin{gathered}
\log \left(-\frac{d \epsilon}{d t}\right) \propto a \cdot \log c^{*}+b \\
\log [\text { unimer }]=-k c^{*} t+b
\end{gathered}
$$

Eq. 15

In the above equations, $c^{*}$ is the concentration of the seed micelles. 
Living CDSA for $\left[\mathbf{P T}{ }_{50} \mathbf{P}\right] \mathbf{I}$ in benzonitrile and THF mixture (THF:benzonitirle $=5: 5$ ).

a
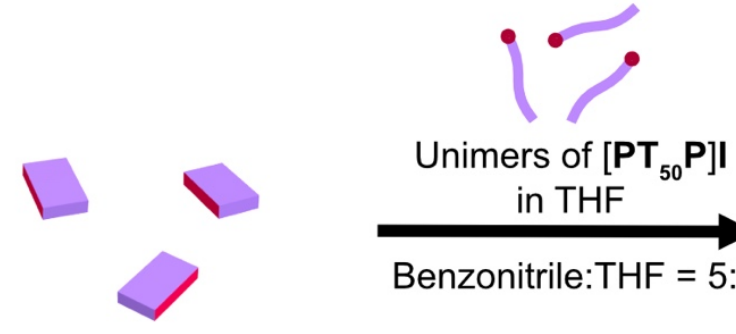

Seed micelles

Benzonitrile: $\mathrm{THF}=5: 5$

\section{Seed micelles}

b

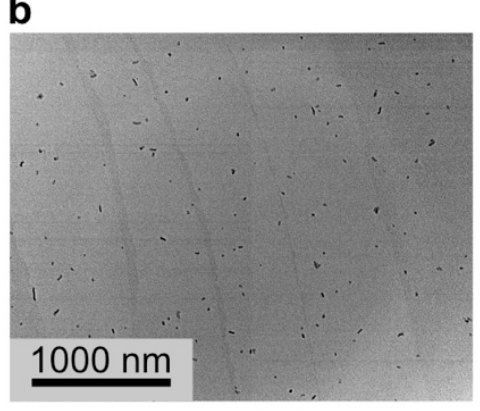

e

C
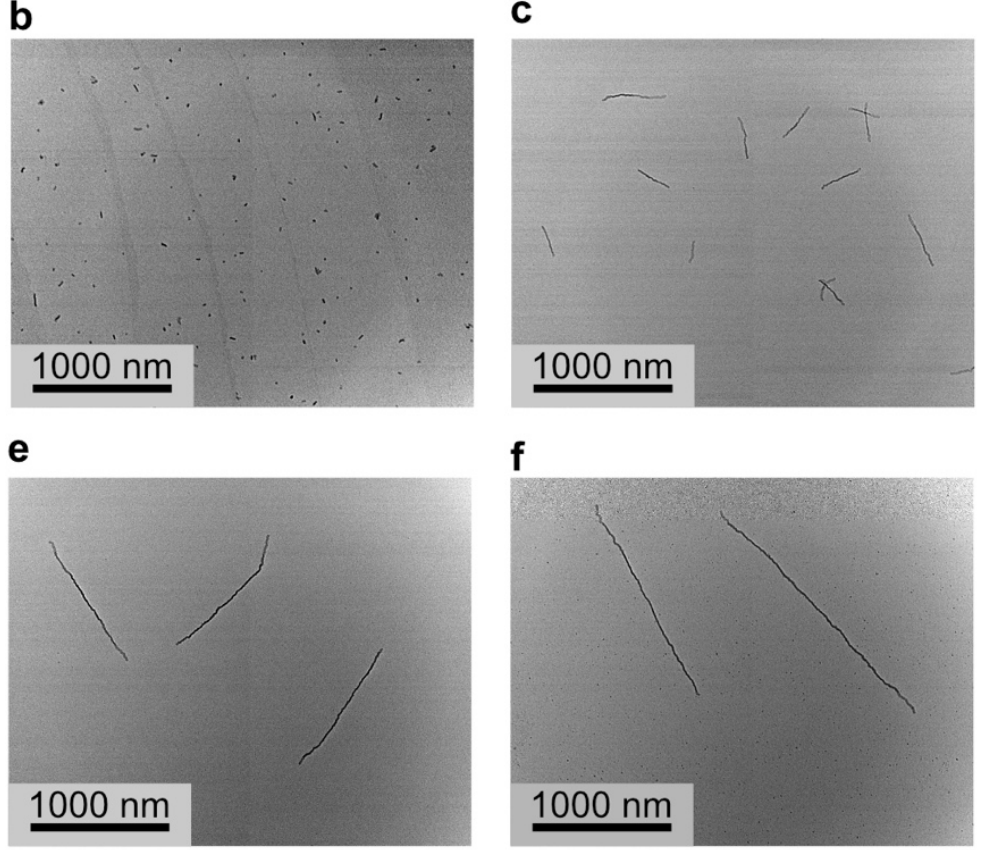

f

$1000 \mathrm{~nm}$

Fiber-like micelles

d

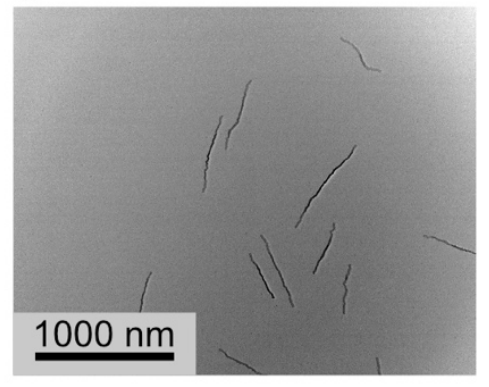

g
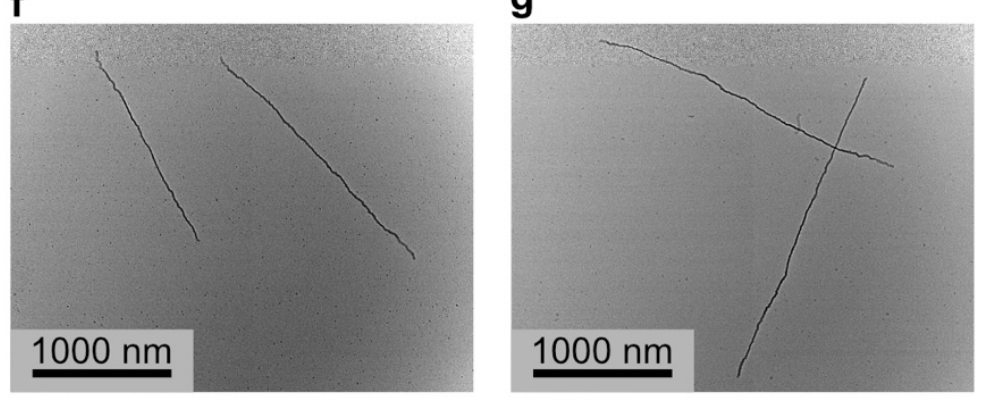

h
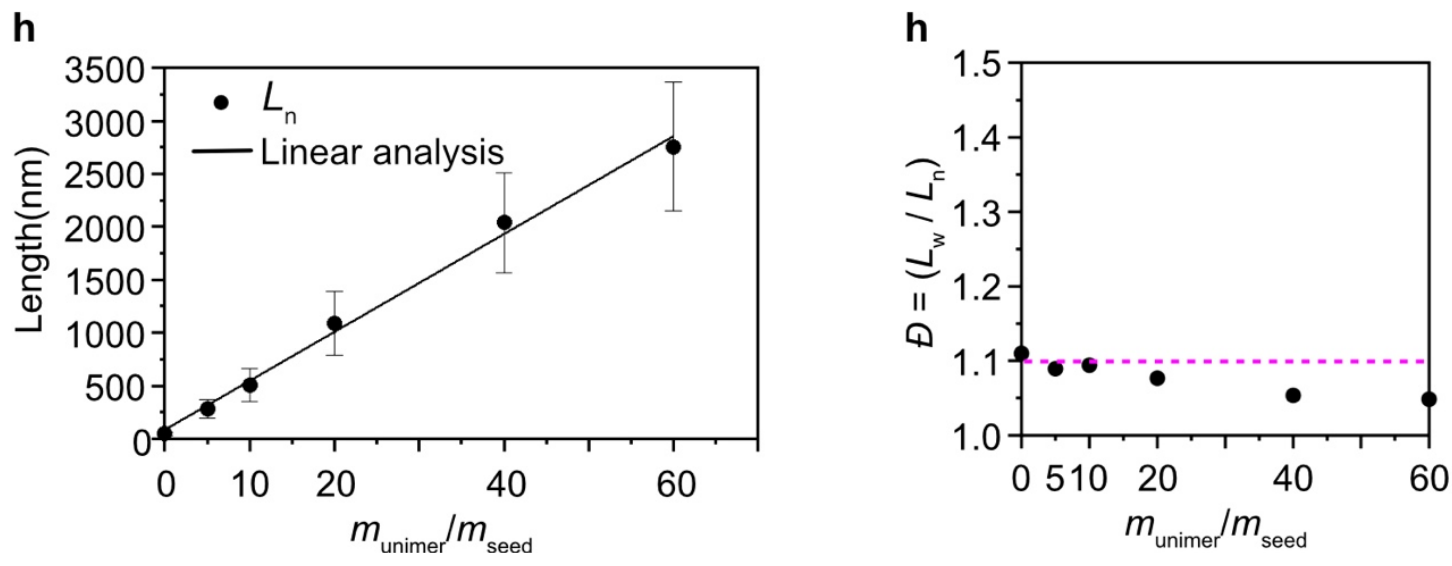

Figure S34.

(a) Schematic representation of living CDSA under the solvent condition of 50 vol. \% of THF in benzonitrile. (b-g) TEM images of length-controlled micelles of $\left[\mathbf{P} \mathbf{T}_{\mathrm{s0}} \mathbf{P}\right] \mathbf{I}$; unimer to seed ratio $m_{\text {unimes }} / m_{\text {sed }}=0(\mathbf{b}), 5(\mathbf{c}), 10(\mathbf{d}), 20(\mathbf{e}), 40(\mathbf{f})$, and $60(\mathbf{g})$. (h) Number-average length $\left(L_{\mathrm{n}}\right)$ of micelles of $\left[\mathbf{P} \mathbf{T}_{\mathrm{s} 0} \mathbf{P}\right] \mathbf{I}$ vs unimer to seed ratio $\left(m_{\text {unines }} / m_{\text {sea }}\right)$. Error bars derived from $\pm \sigma$ (standard deviation) for the micelle length distribution. (i) Polydispersity index $(\nexists)$ of micelles of $\left[\mathbf{P} \mathbf{T}_{\mathrm{s} 0} \mathbf{P}\right] \mathbf{I}$ vs unimer to seed ratio $\left(m_{\text {unima }} / m_{\text {sed }}\right)$. 
$\underline{\text { Table S6: TEM analysis for the living CDSA of [PT }} \underline{\mathbf{5 0}} \underline{\mathbf{P}] \mathbf{I}}$

\begin{tabular}{|c|c|c|c|c|}
\hline$m_{\text {unimer }} / m_{\text {seed }}$ & $L_{\mathrm{n}}(\mathrm{nm})$ & $L_{\mathrm{w}}(\mathrm{nm})$ & $\boldsymbol{\oplus}$ & $\sigma(\mathbf{n m})$ \\
\hline $\mathbf{0}$ & 45.2 & 50.1 & 1.11 & 15 \\
\hline 5 & 284 & 310 & 1.09 & 85 \\
\hline 10 & 504 & 551 & 1.09 & 155 \\
\hline 20 & 1089 & 1172 & 1.08 & 301 \\
\hline 40 & 2040 & 2149 & 1.05 & 472 \\
\hline 60 & 2761 & 2895 & 1.05 & 606 \\
\hline
\end{tabular}


Statistical analysis for the histogram on the length of resulting micelles (THF:beozonitirle $=$ 5:5).

a

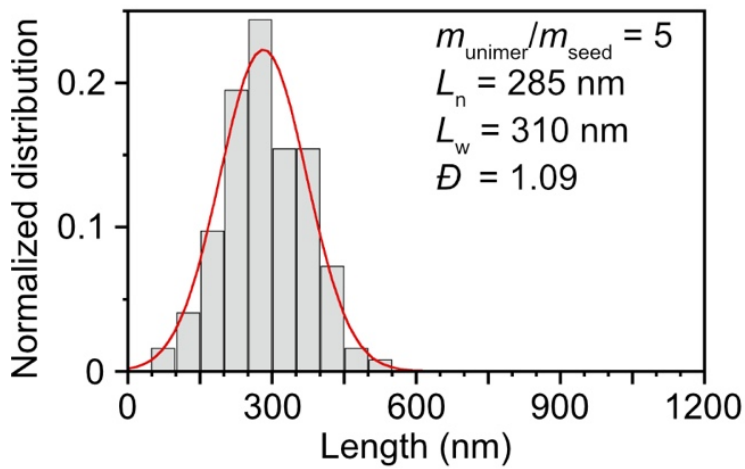

C

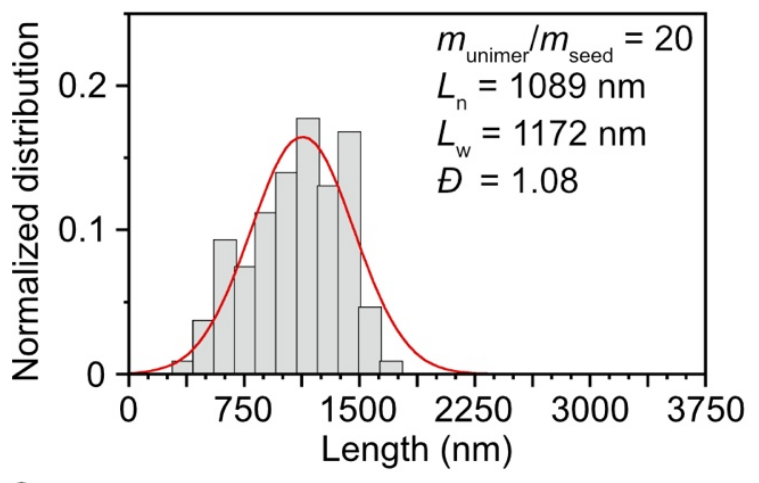

e

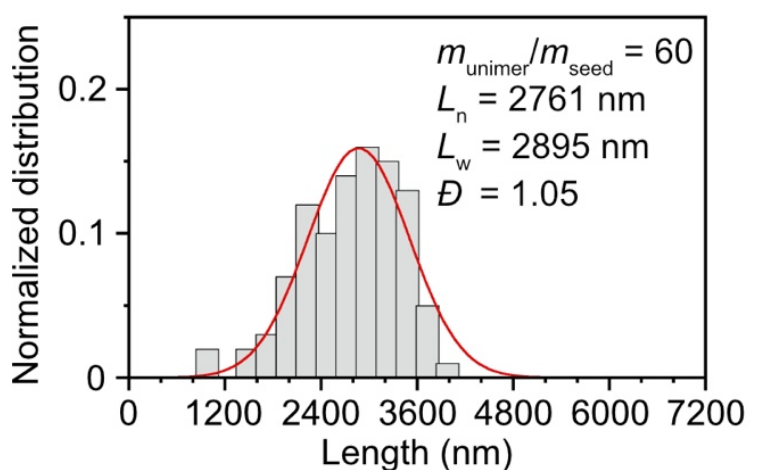

b

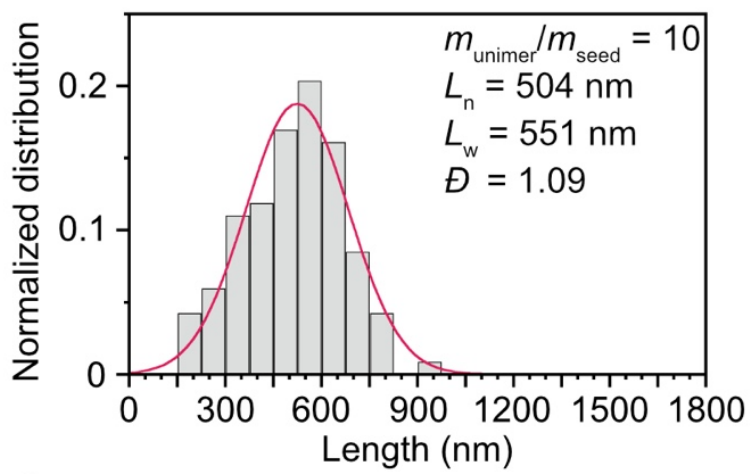

d

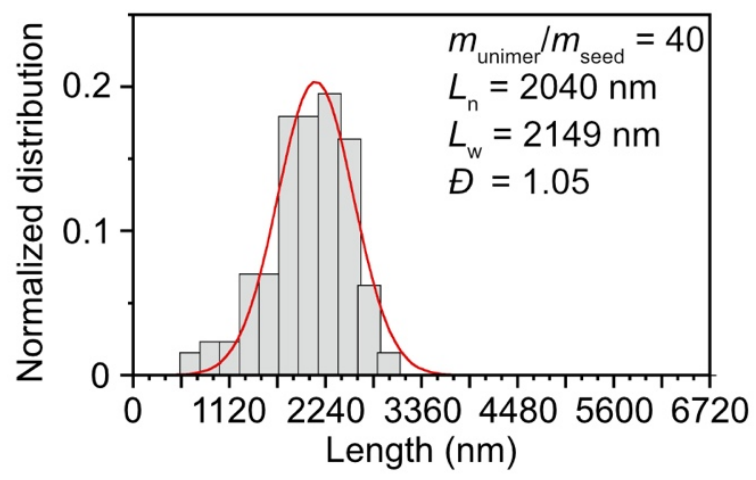

Figure S35.

Histogram of the length of resulting micelles formed by living CDSA (number-average length of seed micelles was $45 \mathrm{~nm}$ as shown in Figure S34): unimer to seed ratio $\left(m_{\text {unimer }} / m_{\text {seed }}\right)=(\mathbf{a})$ 5, (b) 10, (c) 20, (d) 40, and (e) 60. Red lines were the results of the fitting based on gaussian function (Eq. 4).

$$
f(x)=a \cdot \exp \left\{-\frac{\left(x-L_{A V G}\right)^{2}}{2 \sigma^{2}}\right\}
$$


Table S7: The statistical analysis on the histogram of $\left[\mathbf{P T} \underline{\mathbf{5 0}_{\mathbf{0}}} \underline{\mathbf{P}}\right] \mathbf{I}$.

\begin{tabular}{|c|c|c|c|}
\hline $\boldsymbol{m}_{\text {unimer }} \boldsymbol{m}_{\text {seed }}$ & $\boldsymbol{R}^{\mathbf{2}}$ & $\boldsymbol{L}_{\text {avg }}(\mathbf{n m})$ & $\boldsymbol{\sigma}(\mathbf{n m})$ \\
\hline $\mathbf{5}$ & 0.961 & 282 & 90.2 \\
\hline $\mathbf{1 0}$ & 0.953 & 525 & 160 \\
\hline $\mathbf{2 0}$ & 0.900 & 1130 & 335 \\
\hline $\mathbf{4 0}$ & 0.964 & 2127 & 442 \\
\hline $\mathbf{6 0}$ & 0.938 & 2871 & 634 \\
\hline
\end{tabular}




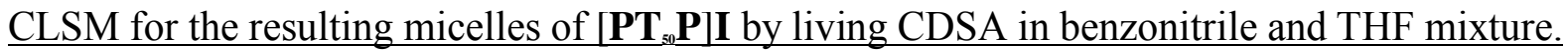

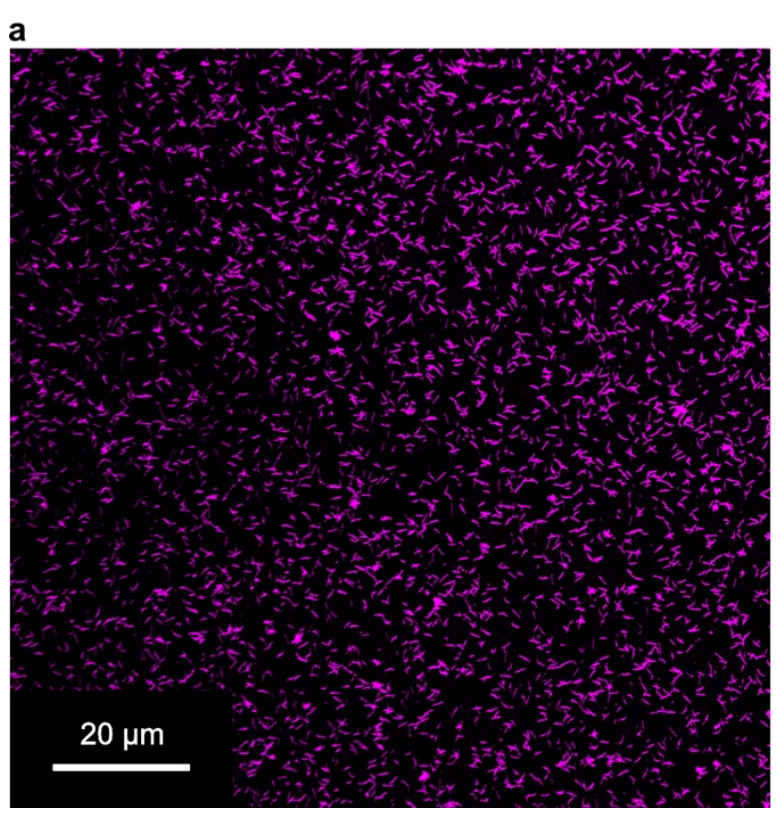

b

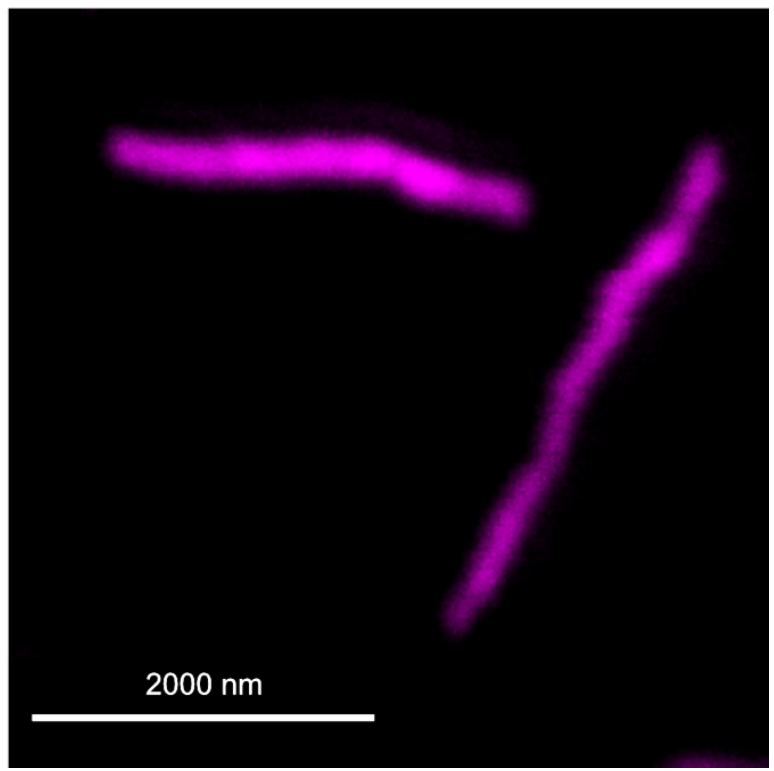

Figure S36.

CLSM images for the fiber-like micelles formed by living CDSA in benzonitrile and THF mixture (benzonitrile: $\mathrm{THF}=5: 5$, Conc.$=0.02 \mathrm{mg} / \mathrm{mL}$ ). 
Stability of fiber-like micelles for the chemical oxidation by iodine.

a

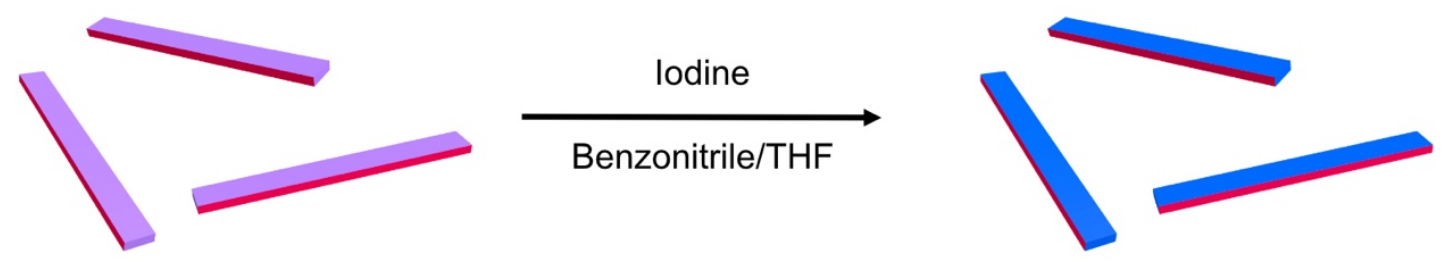

Fiber-like micelles of $\left[\mathrm{PT}_{30} \mathrm{P}\right] \mathrm{I}$

Oxidized fiber-like micelles of $\left[\mathrm{PT}_{30} \mathrm{P}\right] \mathrm{I}$

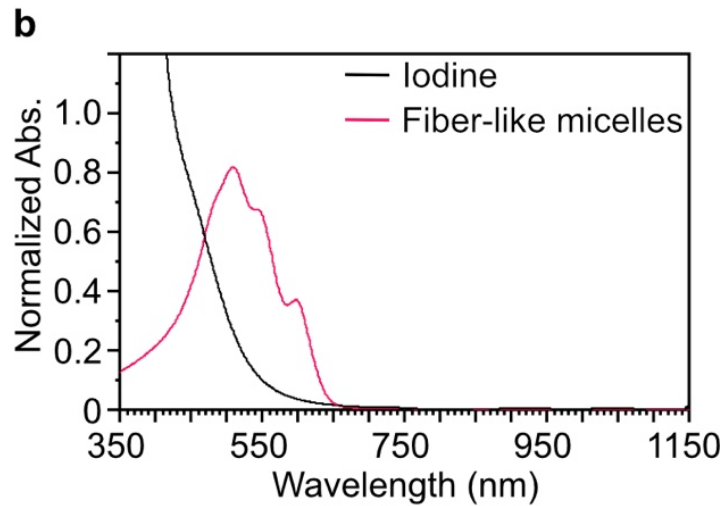

d

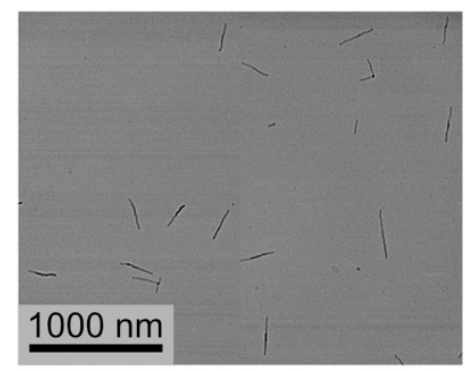

e

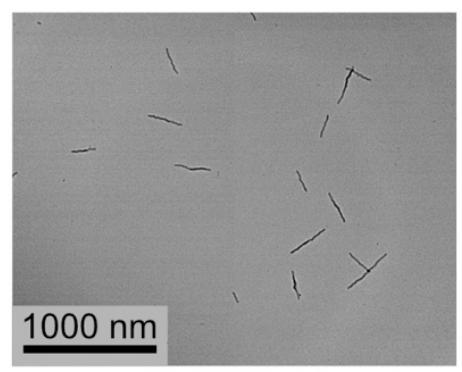

C

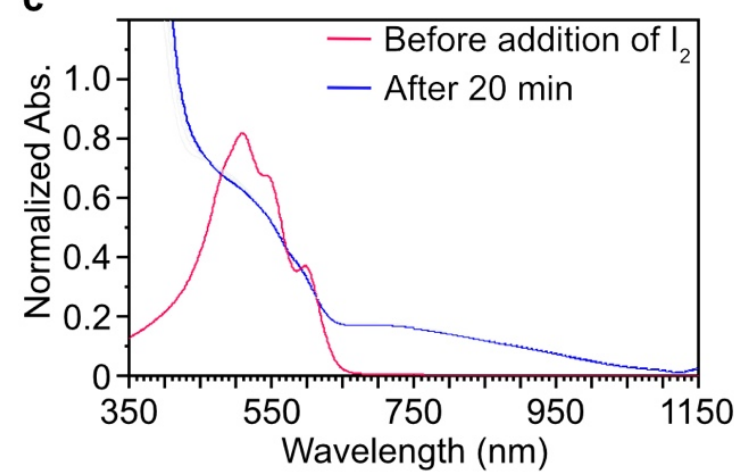

f

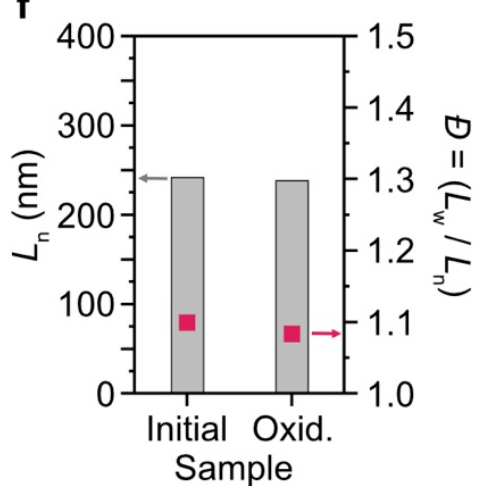

Figure S37.

(a) Schematic image of the chemical oxidation of fiber-like micelles by addition of iodine. (b) Absorption spectra of fiber-like micelles of $\left[\mathbf{P T}_{\mathbf{3 0}} \mathbf{P}\right] \mathbf{I}$ in benzonitrile and THF mixture (pink, Conc. $=0.02 \mathrm{mg} / \mathrm{mL}$, benzonitrile:THF $=9: 1)$ ) or iodine (black, Conc. $=153 \mu \mathrm{M})$. $(\mathbf{c})$ Timecourse of absorption spectra of fiber-like micelles of $\left[\mathbf{P T} \mathbf{~}_{\mathbf{3 0}} \mathbf{P}\right] \mathbf{I}$ in benzonitrile and THF mixture (Conc. $=0.02 \mathrm{mg} / \mathrm{mL}=3.7 \mu \mathrm{M}$, benzonitrile:THF $=9: 1$, Vol. $=3 \mathrm{~mL}, 1$ equiv.) after the addition of iodine solution in benzonitrile (Conc. $=5.0 \mathrm{mM}$, Vol. $=0.12 \mathrm{~mL}, 54$ equiv). The spectra were recorded every $5 \mathrm{~min}$. (d and e) TEM images of the fiber-like micelles (d) before and (e) after the addition of iodine solution in benzonitrile. (f) Number-average length $\left(L_{n}\right.$, left) and dispersity (= $\doteq$, right) of fiber-like micelles before ("Initial") and after ("Oxid") addition of chemical oxidation by iodine. 
Loading gold nanoparticles to the fiber-like micelles through electrostatic interactions.

a

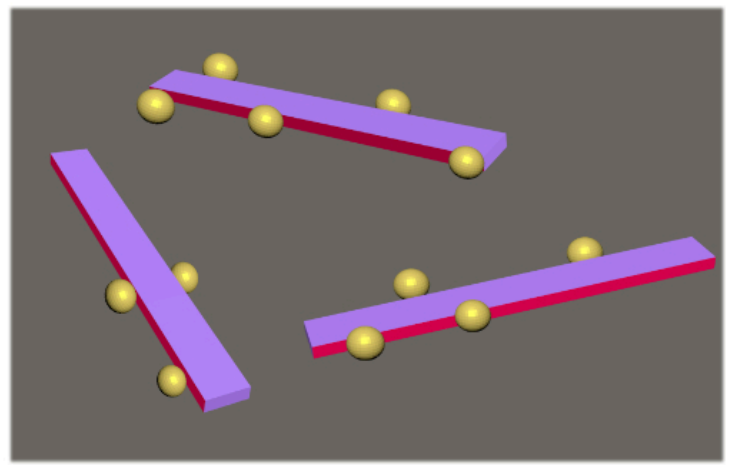

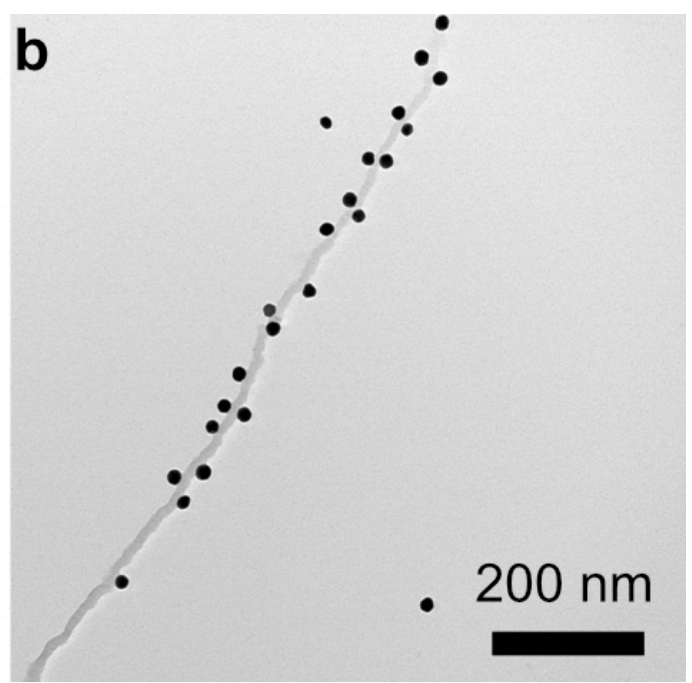

\section{Figure S38.}

(a) Schematic image of the fiber-like micelles on a carbon film after loading the gold nanoparticles. (b) TEM images of the selective loading of the negatively-charged gold nanoparticles (citrate-stabilized gold nanoparticles with the diameter of $20 \mathrm{~nm}$ ) to fiber-like micelles $\left(\mathrm{DP}_{\mathrm{n}}=30, L_{\mathrm{n}}=465 \mathrm{~nm}, \emptyset=1.11\right)$. The fiber-like micelles were dispersed on the carbon-coated $\mathrm{Cu}$ grid and immersed in the solution of gold nanoparticles in propan-2-ol and water overnight. Then, the grid was washed by water, methanol, and acetone to wash out the free gold nanoparticles on the grid. The attachment of the negatively-charged gold nanoparticles is consistent with a preferred antiparallel arrangement of the cationic chargeterminated P3HT amphiphiles. 


\section{References}

S1. Biancalana, L., Zacchini, S., Ferri. N., Lupo, M. G., Pampaloni, G. \& Marchetti, F. Tuning the cytotoxicity of ruthenium(ii) para-cymene complexes by mono-substitution at a triphenylphosphine/phenoxydiphenylphosphine ligand. Dalton Trans. 2017, 46, 16589-16604.

S2. Dudenko, D., Kiersnowski, A., Shu, J., Pisula, W., Sebastiani, D., Spiess, H. W. \& Hansen, M. R. A strategy for revealing the packing in semicrystalline $\pi$-conjugated polymers: Crystal structure of bulk poly-3-hexyl-thiophene (P3HT). Angew. Chem. Int. Ed. 2012, 51, 11068-11072.

S3. Zhao, D. \& Moore, J. S. Nucleation-Elongation Polymerization under Imbalanced Stoichiometry. J. Am. Chem. Soc. 2003, 125, 16294-16299.

S4. Ogi, S., Sugiyasu, K., Manna, S., Samitsu, S. \& Takeuchi, M. Living supramolecular polymerization realized through a biomimetic approach. Nat. Chem. 2014, 6, 188-195.

S5. Ogi, S., Stepanenko, V., Sugiyasu, K., Takeuchi, M. \& Würthner, F. Mechanism of selfassembly process and seeded supramolecular polymerization of perylene bisimide organogelator. J. Am. Chem. Soc. 2015, 137, 3300-3307.

S6. Fukui, T., Kawai, S., Fujinuma, S., Matsushita, Y., Yasuda, T., Sakurai, T., Seki, S., Takeuchi, M. \& Sugiyasu, K. Control over differentiation of a metastable supramolecular assembly in one and two dimensions. Nat. Chem. 2017, 9, 493-499. 